\title{
A Microdosimetric Analysis of the Interactions of Mono-Energetic Neutrons with Human Tissue
}

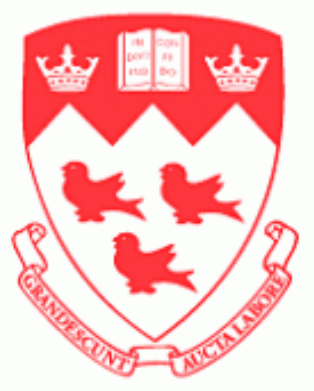

A thesis submitted to McGill University in partial fulfilment of the requirements for the degree of Master of Science in Medical Physics, August 2019

(C) Christopher Lund, 2019 



\section{Declaration of Authorship}

I, Christopher M. Lund, declare that this thesis titled, "A Microdosimetric Analysis of the Interactions of Mono-Energetic Neutrons with Human Tissue" and the work presented in it are my own. I confirm that:

- This work was done wholly or mainly while in candidature for a research degree at this university.

- Where any part of this thesis has previously been submitted for a degree or any other qualification at this University or any other institution, this has been clearly stated.

- Where I have consulted the published work of others, this is always clearly attributed.

- Where I have quoted from the work of others, the source is always given. With the exception of such quotations, this thesis is entirely my own work.

- I have acknowledged all main sources of help.

- Where the thesis is based on work done by myself jointly with others, I have made clear exactly what was done by others and what I have contributed myself. 



\section{Abstract}

Nuclear reactions induced during high-energy radiation therapy produce secondary neutrons that, due to their carcinogenic potential, constitute an important risk for the development of iatrogenic cancer. Experimental and epidemiological findings indicate a marked energy dependence of neutron relative biological effectiveness (RBE) for carcinogenesis, but little is known about its physical basis. While the exact mechanism of radiation carcinogenesis is yet to be fully elucidated, numerical microdosimetry can be used to predict the biological consequences of a given irradiation based on its microscopic pattern of energy depositions. Building on recent work, this thesis studies the physics underlying neutron RBE by using the microdosimetric quantity dose-mean lineal energy $\left(\bar{y}_{D}\right)$ as a proxy.

A simulation pipeline was constructed to explicitly calculate the $\bar{y}_{D}$ of radiation fields that consists of (i) the open source Monte Carlo (MC) toolkit Geant4, (ii) its radiobiological extension Geant4-DNA, and (iii) a weighted track-sampling algorithm. This approach was used to evaluate the $\bar{y}_{D}$ of mono-energetic neutrons with initial kinetic energies between $1 \mathrm{eV}$ and $10 \mathrm{MeV}$ at multiple depths in a tissue-equivalent phantom approximately the size of a human adult torso. Spherical sampling volumes with diameters between $2 \mathrm{~nm}$ and $1 \mu \mathrm{m}$ were considered. To obtain a measure of RBE, the neutron $\bar{y}_{D}$ values were divided by those of $250 \mathrm{keV}$ x-rays that were calculated in the same way. Qualitative agreement was found with published radiation protection factors and simulation data, allowing for the dependencies of neutron RBE on depth and energy to be discussed in the context of the neutron interaction cross sections and secondary particle distributions in human tissue. 



\section{Résumé}

Les réactions nucléaires induites par la radiothérapie à haute énergie produisent des neutrons secondaires qui, en raison de leur potentiel cancérogène, constituent un risque important pour le développement du cancer iatrogène. Les résultats expérimentaux et épidémiologiques indiquent une importante dépendance énergétique de l'efficacité biologique relative (EBR) des neutrons pour la cancérogenèse, mais on en connait toutefois très peu sur ses fondements physiques. Bien que le mécanisme exact de la cancérogenèse par rayonnement n'ait pas encore été complètement élucidé, la microdosimétrie numérique peut être utilisée pour prédire les conséquences biologiques d'une irradiation donnée sur la base de son modèle microscopique de dépôts d'énergie. En s'appuyant sur des travaux récents, cette thèse étudie la physique sous-jacente à l'EBR des neutrons en utilisant la dose-énergie moyenne linéaire $\left(\bar{y}_{D}\right)$, une quantité microdosimétrique, comme proxy.

Un structure de simulation a été développée pour calculer explicitement la $\bar{y}_{D}$ des champs de rayonnement. Elle est constituée de (i) la boîte à outils en libre accès Monte Carlo (MC) Geant4, (ii) de son extension radiobiologique Geant4-DNA, et (iii) d'un algorithme d'échantillonnage pondéré. Cette approche a été utilisée pour évaluer la $\bar{y}_{D}$ de neutrons mono-énergétiques, dont l'énergie cinétique initiale était comprise entre $1 \mathrm{eV}$ et $10 \mathrm{MeV}$, à plusieurs profondeurs dans un fantôme de composition équivalente aux tissus biologiques et de taille comparable à torse humain adulte. Des volumes d'échantillonnage de forme sphérique ont été considérés, avec un diamètre compris entre $2 \mathrm{~nm}$ et $1 \mathrm{\mu m}$. Pour obtenir une mesure de l'EBR, les valeurs de $\bar{y}_{D}$ de neutrons ont été divisées par celles de rayons $\mathrm{X}$ de $250 \mathrm{keV}$, calculées de la même manière. Un accord qualitatif a été établi entre les facteurs de radioprotection publiés et les données de simulation. Cela a permis un discussion sur les dépendances de l'EBR sur la profondeur et l'énergie sont discutées dans le contexte des sections efficaces d'interaction neutronique et de la distribution des particules secondaires dans les tissus humains. 



\section{Acknowledgements}

To start, I would like to thank my supervisor Dr. John Kildea for his support and guidance towards my improvement as a researcher. I'd also like to give a big thank you to the members of the NICE-ROKS group: Logan for the many hours of discussion and editing, Haley for all her help, Felix for the coding implementation debates, and Kelly for that story about his Spotify account. This project would also not have been possible without Gabe's advice on methodology and experiment design. To Piotr, Jan, and Shirin for useful discussions and comments, and to Julien for translation and editing help, thank you. Finally, I would not be here without the love and support of my parents, Morgan, and the rest of my family. 



\section{Contents}

Declaration of Authorship iii

Abstract $\quad$ v

Résumé vii

Acknowledgements $\quad$ ix

1 Introduction $\quad 1$

1.1 Thesis overview . . . . . . . . . . . . . . . . . . 1

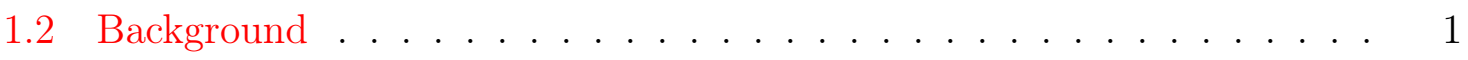

1.2 .1 Cancer ......................... 1

1.2 .2 Ionizing radiation . . . . . . . . . . . . . . . . 2

1.2.3 Radiation therapy . . . . . . . . . . . . . . . . . 2

Brachytherapy . . . . . . . . . . . . . 3

External beam radiation therapy . . . . . . . . . . . 3

1.2.4 Radiation carcinogenesis . . . . . . . . . . . . . . . 4

1.2.5 Numerical methods in radiation carcinogenesis studies . . . . . 5

2 Radiation Physics $\quad 7$

2.1 Types of ionizing radiation and their interactions . . . . . . . . 7

2.1.1 Photons ..................... 7

Rayleigh scattering . . . . . . . . . . . . . 8

Photoelectric effect . . . . . . . . . . . . . 8

Compton scattering . . . . . . . . . . . . . . 8

Pair and triplet production . . . . . . . . . . . . . 9

Photoneutron production . . . . . . . . . . . . . . 9

Characteristic x-rays and the Auger effect . . . . . . . . . . . 9

2.1.2 Charged particles . . . . . . . . . . . . . . 10

Particle-orbital electron interactions . . . . . . . . . . . . 10

Particle-nucleus interactions . . . . . . . . . . . . . . 11

Stopping power and range . . . . . . . . . . . . . . . 11

Linear energy transfer . . . . . . . . . . . . . . 13 
2.1 .3 Neutrons. . . . . . . . . . . . . . . . . . . . . . . . . 13

Classification . . . . . . . . . . . . . . 13

Elastic scattering . . . . . . . . . . . . . . . . 14

Inelastic scattering . . . . . . . . . . . . . . . . . 14

Neutron capture . . . . . . . . . . . . . . . . 14

Nuclear spallation . . . . . . . . . . . . . . . 15

Neutron-induced fission . . . . . . . . . . . . . . . 15

2.2 The Monte Carlo method _ . . . . . . . . . . . . . . . . 15

2.2 .1 Radiation transport with $\mathrm{MC} \ldots \ldots \ldots \ldots$

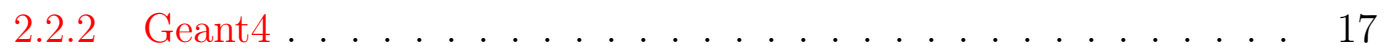

Radiation transport in Geant4 . . . . . . . . . . . . . . 18

Geant4-DNA . . . . . . . . . . . . . . . 19

3 Radiobiology 21

3.1 The genome . . . . . . . . . . . . . . . . . . 21

3.1 .1 The DNA molecule . . . . . . . . . . . . . . . . . . 21

3.1 .2 Higher order structure $\ldots \ldots \ldots \ldots . \ldots \ldots 22$

3.1 .3 Codons and the gene . . . . . . . . . . . . . . . 23

3.2 The biological action of ionizing radiation $\ldots \ldots \ldots \ldots \ldots$

3.2 .1 Direct vs. indirect action . . . . . . . . . . . . . . 23

3.2 .2 Radiation-induced DNA damage and repair . . . . . . . 25

3.3 Important quantities and concepts in dosimetry and radiation safety . . 26

3.3 .1 Physical quantities . . . . . . . . . . . . . . . 26

Fluence and flux . . . . . . . . . . . . . . . 26

Kerma . . . . . . . . . . . . . . . . . . . . 28

3.3.2 Dosimetric quantities and concepts . . . . . . . . . . . . 29

Absorbed dose . . . . . . . . . . . . . . . . . . . . . . 29

Charged particle equilibrium . . . . . . . . . . . . . . . . . 29

RBE . . . . . . . . . . . . . . . . 30

Equivalent dose . . . . . . . . . . . . . . . . 31

Effective dose . . . . . . . . . . . . . . 31

4 Microdosimetry 33

4.1 Important quantities and concepts . . . . . . . . . . . . . . . 33

4.1.1 The inchoate distribution and its intersection with sampling volumes . . . . . . . . . . . . . . . . . . . . . 34

4.1.2 Energy deposit and imparted energy . . . . . . . . . . . . 34

4.1 .3 Lineal energy . . . . . . . . . . . . . . . . . . . . . 35

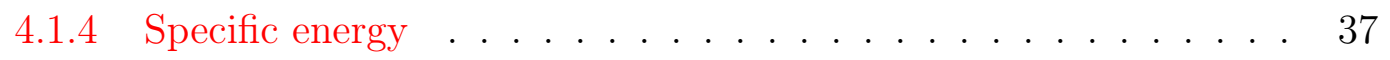


4.2 Track sampling $\ldots \ldots \ldots \ldots \ldots \ldots \ldots$

4.2 .1 General considerations . . . . . . . . . . . . . . . 38

4.2 .2 The weighted track-sampling algorithm . . . . . . . . . 39

4.3 Microdosimetry in radiobiology . . . . . . . . . . . . . . . . 41

5 Methods 45

5.1 Overview of the microdosimetric simulation

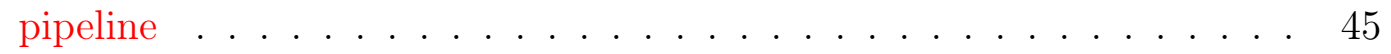

5.2 Condensed history simulations . . . . . . . . . . . . . 46

5.2 .1 Geometry . . . . . . . . . . . . . . . . 46

5.2 .2 Irradiation conditions . . . . . . . . . . . . . . . 47

5.2 .3 Physics. . . . . . . . . . . . . . . . . . . . . . 48

5.2 .4 Scoring . . . . . . . . . . . . . . . . . . . . . . . . 49

Spectra and relative dose . . . . . . . . . . . . . . . 49

Explicit dose calculation . . . . . . . . . . . . . . . . 49

5.3 Track-structure simulations $\ldots \ldots \ldots \ldots$

5.3.1 Geometry and irradiation conditions . . . . . . . . . 49

5.3 .2 Physics. . . . . . . . . . . . . . . . . 50

5.3 .3 Scoring . . . . . . . . . . . . . . . . . . . . . 51

5.4 Final results . . . . . . . . . . . . . . . . . 53

6 Results 55

6.1 Charged particle spectra $\ldots \ldots \ldots \ldots \ldots \ldots \ldots$

6.1 .1 Neutrons. . . . . . . . . . . . . . . . . . 55

$6.1 .2250 \mathrm{keV} \mathrm{x}$-rays . . . . . . . . . . . . . . . . 58

6.2 Relative dose contributions . . . . . . . . . . . . . . . . . . 58

6.3 Dose-mean lineal energies . . . . . . . . . . . . . . . . . . . . 59

6.3.1 Neutron secondaries . . . . . . . . . . . . . . . . . . . 59

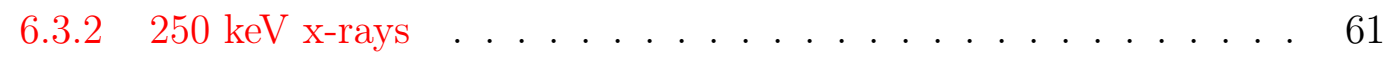

6.4 Neutron $\mathrm{RBE} \ldots \ldots \ldots \ldots \ldots \ldots$

$\begin{array}{lll}7 & \text { Discussion } & 69\end{array}$

7.1 Charged particle spectra . . . . . . . . . . . . . . . . . 69

7.1.1 Neutron-generated spectra . . . . . . . . . . . . . . . 69

Secondary particles . . . . . . . . . . . . . . . . . 69

Higher order electron spectra . . . . . . . . . . . . . 70

$7.1 .2250 \mathrm{keV}$ x-ray secondary particle spectra . . . . . . . . . 72

7.2 Relative dose contributions . . . . . . . . . . . . . . . . . 72

7.3 Dose-mean lineal energies . . . . . . . . . . . . . . . . 74 
xiv

7.4 Neutron RBE . . . . . . . . . . . . . . . . . . . . 76

8 Summary and Conclusions

Bibliography

83 


\section{List of Figures}

1.1 Linac schematic . . . . . . . . . . . . . . . . . . . 4

2.1 Particle collisions . . . . . . . . . . . . . . . . . . . . . 10

2.2 Analogue transport of photons . . . . . . . . . . . . 16

3.1 The DNA strand . . . . . . . . . . . . . . . . . . . . . . . 22

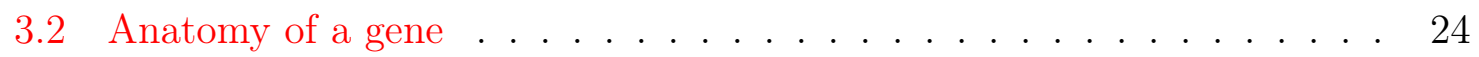

3.3 Direct and indirect action . . . . . . . . . . . . 25

3.4 Non-lethal chromosome aberrations . . . . . . . . . . . . . . 27

3.5 External source $\mathrm{CPE} \ldots \ldots \ldots \ldots \ldots$

3.6 Neutron weighting factors $\ldots \ldots \ldots \ldots \ldots$

4.1 Multi-events . . . . . . . . . . . . . . . . . . . 35

4.2 Associated volume . . . . . . . . . . . . . . . . . . . 39

4.3 Weighted track-sampling . . . . . . . . . . . . . . 40

5.1 Simulation workflow $\ldots \ldots \ldots \ldots \ldots \ldots \ldots$

5.2 Tissue phantom . . . . . . . . . . . . . . . . . . . 48

6.1 Spectra of neutron secondaries . . . . . . . . . . . 56

6.2 Electron spectra . . . . . . . . . . . . . . . . . . . 57

6.3 Spectra of x-ray secondaries $\ldots \ldots \ldots \ldots \ldots \ldots$

6.4 Relative dose contributions . . . . . . . . . . . . . . . 60

$6.5 \bar{y}_{D}$ of neutron secondaries $\ldots \ldots \ldots \ldots \ldots \ldots \ldots \ldots \ldots \ldots$

6.6 Sampling diameter effect on proton $\bar{y}_{D} \ldots \ldots \ldots \ldots \ldots$

6.7 Sampling diameter effect on electron $\bar{y}_{D} \ldots \ldots \ldots \ldots$. . . . . . 64

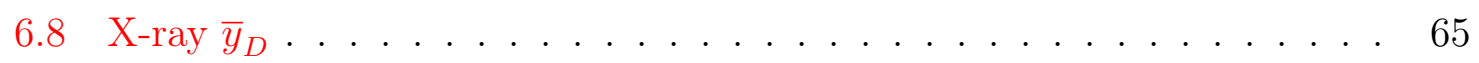

6.9 Neutron RBE . . . . . . . . . . . . . . . . . . . . 67

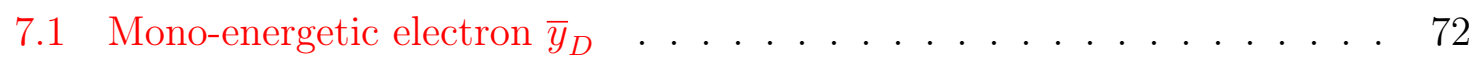

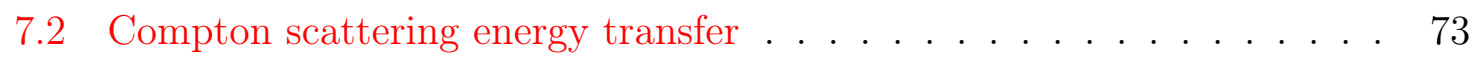

$7.3 \bar{y}_{D}$ of recoil protons $\ldots \ldots \ldots \ldots \ldots \ldots \ldots \ldots \ldots$

7.4 Nitrogen capture cross section . . . . . . . . . . . . . . 76

7.5 Alpha particle spectra . . . . . . . . . . . . . . 77 

xvii

\section{List of Tables}

2.1 Neutron energy classification . . . . . . . . . . . . . . . . 13

5.1 Composition of the ICRU-4 sphere . . . . . . . . . . . . . . . 47

5.2 Electron transport models . . . . . . . . . . . . . . . . 51

5.3 Proton and hydrogen transport models . . . . . . . . . . . . . . . . 52

5.4 Helium transport models . . . . . . . . . . . . . . . . . . . . . 52 



\section{List of Abbreviations}

$\begin{array}{ll}\text { ALARA } & \text { As Low As Reasonably Achievable } \\ \text { CH } & \text { Condensed History monte carlo } \\ \text { CPE } & \text { Charged Particle Equilibrium } \\ \text { CSDA } & \text { Continuous Slowing Down Approximation } \\ \text { DNA } & \text { DeoxyriboNucleic Acid } \\ \text { DSB } & \text { Double Strand Break } \\ \text { EBRT } & \text { External Beam Radiation Therapy } \\ \text { GEANT4 } & \text { GEometry ANd Tracking } 4 \\ \text { GHEISHA } & \text { Gamma, Hadron, and Electron Interaction and SHower Algorithm } \\ \text { GPS } & \text { General Particle Source } \\ \text { IAEA } & \text { International Atomic Energy Agency } \\ \text { ICRP } & \text { International Commission on Radiological Protection } \\ \text { ICRU } & \text { International Commission on Radiation Units and measurements } \\ \text { ICRU-4 } & \text { ICRU 4-component soft tissue-equivalent sphere } \\ \text { KERMA } & \text { Kinetic Energy Released per unit MAss } \\ \text { LET } & \text { Linear Energy Transfer } \\ \text { LINAC } & \text { LINear ACcelerator } \\ \text { LMDS } & \text { Locally Multiply Damaged Sites } \\ \text { LNT } & \text { Linear-No-Threshold } \\ \text { MC } & \text { Monte Carlo } \\ \text { MFP } & \text { Mean Free Path } \\ \text { NIST } & \text { National Institute of Standards and Technology } \\ \text { PHITS } & \text { Particle and Heavy Ion Transport code System } \\ \text { RBE } & \text { Relative Biological Effectiveness } \\ \text { SSB } & \text { Single Strand Break } \\ \text { TDRA } & \text { Theory of Dual Radiation Action } \\ \text { TS } & \text { Track-Structure (monte carlo) } \\ \text { US NRC } & \text { United States Nuclear Regulatory Commission } \\ & \end{array}$





\section{Physical Constants}

Avogadro's constant

Electron rest mass

Elementary charge

Neutron rest mass

Planck's constant

$\mathrm{Pi}$

Speed of light in a vacuum

Unified atomic mass unit

$$
\begin{aligned}
N_{A} & =6.022 \times 10^{23} \mathrm{~mol}^{-1} \\
m_{e} & =9.109 \times 10^{-31} \mathrm{~kg} \\
e & =1.602 \times 10^{-19} \mathrm{C} \\
m_{n} & =1.675 \times 10^{-27} \mathrm{~kg} \\
h & =6.626 \times 10^{-34} \mathrm{~J} \mathrm{~s} \\
\pi & =3.14159 \ldots \\
c & =2.998 \times 10^{8} \mathrm{~m} \mathrm{~s}^{-1} \\
\mathrm{u} & =1.661 \times 10^{-27} \mathrm{~kg}
\end{aligned}
$$





\section{List of Symbols}

a outer radius of an atom

$\mathrm{m}$

$A \quad$ atomic mass number

A cross-sectional area

$\mathrm{m}^{2}$

b impact parameter

$\mathrm{m}$

D absorbed dose

Gy $\left(\mathrm{J} \mathrm{kg}^{-1}\right)$

$E \quad$ effective dose

$\mathrm{Sv}$

$E \quad$ energy

$\mathrm{J}$

$E_{a b s}$

absorbed energy

$\mathrm{J}$

$E_{B} \quad$ binding energy

$\mathrm{J}$

$E_{t r} \quad$ energy transferred

$\mathrm{J}$

$E_{K} \quad$ kinetic energy

$\mathrm{J}$

$\bar{g} \quad$ radiative fraction

$H_{T} \quad$ equivalent dose

Sv

K kerma

Gy

$\dot{K} \quad$ kerma rate

Gy s $^{-1}$

$K_{e l} \quad$ electronic kerma

Gy

$K_{\text {rad }} \quad$ radiative kerma

Gy

$\bar{\ell} \quad$ mean chord length

$\mathrm{m}$

$L_{\Delta} \quad$ linear energy transfer

$\mathrm{J} \mathrm{m}^{-1}$

m mass

$\mathrm{kg}$

$N \quad$ particle number

$Q \quad$ Q value (change in rest mass)

$\mathrm{J}$

$r \quad$ radius

m

$R \quad$ range

$\mathrm{m}$

$R_{C S D A}$ CSDA range

$\mathrm{m}$

$R_{p} \quad$ practical range

$\mathrm{m}$

$R_{50} \quad 50 \%$ range

$\mathrm{m}$

$s \quad$ path length

$\mathrm{m}$

$S \quad$ surface area

$\mathrm{m}^{2}$

$S \quad$ mass stopping power

$\mathrm{J} \mathrm{m}^{-1}$

$S_{c o l} \quad$ mass collision stopping power

$\mathrm{J} \mathrm{m}^{-1}$ 
xxiv

\begin{tabular}{|c|c|c|}
\hline$S_{\text {rad }}$ & mass radiative stopping power & $\mathrm{J} \mathrm{m}^{-1}$ \\
\hline$V$ & volume & $\mathrm{m}^{3}$ \\
\hline$w_{R}$ & radiation weighting factor & \\
\hline$w_{T}$ & tissue weighting factor & \\
\hline$y$ & lineal energy & $\mathrm{J} \mathrm{m}^{-1}$ \\
\hline $\bar{y}_{D}$ & dose-mean lineal energy & $\mathrm{J} \mathrm{m}^{-1}$ \\
\hline $\bar{y}_{F}$ & frequency-mean lineal energy & $\mathrm{J} \mathrm{m}^{-1}$ \\
\hline$y^{*}$ & saturation-corrected dose-mean lineal energy & $\mathrm{J} \mathrm{m}^{-1}$ \\
\hline$z$ & specific energy & Gy \\
\hline $\bar{z}_{D}$ & dose-mean specific energy & Gy \\
\hline $\bar{z}_{D, s}$ & single event dose-mean specific energy & Gy \\
\hline $\bar{z}_{F}$ & frequency-mean specific energy & Gy \\
\hline $\bar{z}_{F, s}$ & single event frequency-mean specific energy & Gy \\
\hline$Z$ & atomic number & \\
\hline$\gamma$ & gamma-ray & \\
\hline$\epsilon$ & normalized photon energy & $\mathrm{J}$ \\
\hline$\epsilon$ & energy imparted & $\mathrm{J}$ \\
\hline$\epsilon_{i}$ & energy deposit & $\mathrm{J}$ \\
\hline$\theta$ & scattering angle & $\mathrm{rad}$ \\
\hline$\nu$ & frequency & $\mathrm{s}^{-1}$ \\
\hline$\rho$ & density & $\mathrm{kg} \mathrm{m}^{-3}$ \\
\hline$\phi$ & recoil angle & $\mathrm{rad}$ \\
\hline$\Phi$ & fluence & $\mathrm{m}^{-2}$ \\
\hline $\bar{\Phi}$ & fluence (MC) & $\mathrm{m}^{-2}$ \\
\hline$\dot{\Phi}$ & fluence rate & $\mathrm{m}^{-2} \mathrm{~s}^{-1}$ \\
\hline$\Phi_{E}$ & fluence spectrum & $\mathrm{m}^{-2}$ \\
\hline$\omega$ & angular frequency & $\mathrm{rad}$ \\
\hline$\Psi$ & energy fluence & $\mathrm{J} \mathrm{m}^{-2}$ \\
\hline$\dot{\Psi}$ & energy flux & $\mathrm{J} \mathrm{m}^{-2} \mathrm{~s}^{-}$ \\
\hline
\end{tabular}




\section{Chapter 1}

\section{Introduction}

\subsection{Thesis overview}

This thesis describes the implementation and use of a microdosimetric weighted tracksampling algorithm for the study of neutron energy deposition patterns in human tissue. Using a microdosimetric endpoint as a proxy for the biologic effects of neutrons, the primary findings of this thesis are compared to experimentally-derived risk factors for neutron carcinogenesis as well as to recent results from similar studies.

In the remainder of this chapter, an overview of the relationships between radiation and cancer is given in the context of radiation therapy and radiation safety. Chapter 2 summarizes the theory behind the primary interactions of ionizing radiation with matter and how they can be modelled numerically. The progression from initial radiation insult to the induction of cancer is then expanded upon in Chapter 3 along with the definition of some quantities used to specify the amount of radiation interaction with matter. An overview of the theory of microdosimetry, relevant numerical techniques, and the application of microdosimetry to radiobiology and neutron carcinogenesis is given in Chapter 4. Chapter 5 describes details of the simulation workflow and code implementation, while Chapter 6 presents the results. A discussion of these results is given in Chapter 7. Finally, conclusions and an outline of future studies are given in Chapter 8 .

\section{$1.2 \quad$ Background}

\subsubsection{Cancer}

Cancer is a large and diverse set of genetic diseases characterized by the uncontrolled proliferation of cells. This growth often leads to the formation of masses of abnormal cells. Referred to as tumours, these masses may stay in place or spread into nearby healthy tissues. The first type, referred to as benign tumours, often present a low risk of harm to the patient. The second, however, are malignant, or cancerous, present a 
significant health risk to the patient and are more difficult to treat. Cancerous tumours may spread far from their location of origin by spawning new growths in a process called metastasis. As the primary cancerous tumour and its metastases (secondary masses) grow and invade areas of healthy tissue, they may disrupt the normal function of organs and organ systems within living organisms. Eventually, these systems may shutdown and, as a result, the disease becomes fatal.

Unfortunately, cancer is prevalent among human populations. Approximately half of Canadians will be diagnosed with some form of cancer during their lifetime and about $50 \%$ of these will die from it, making cancer the leading cause of death in Canada [1]. As such, the detection, diagnosis, and treatment of cancer feature heavily in scientific and medical efforts. This has led to increasing 5-year survival rates and decreasing incidence rates; however, the ageing population of Canada has nonetheless resulted in an ever-increasing number of cancer diagnoses [1]. Furthermore, it has led to an ever-increasing number of cancer survivors living with the side-effects of their treatments [2].

The treatment of a particular cancer depends upon its specific type, stage (how advanced it is), size, and a variety of factors related to the individual patient. It is sometimes sufficient to simply remove the tumour(s) surgically. Cancer-fighting drugs may also be used to either kill the cancerous cells, activate the patient's immune system to attack them, or suppress their growth signals. Finally, many patients receive some form of radiation therapy, either alone or in conjunction with the other treatment modalities.

\subsubsection{Ionizing radiation}

Radiation refers to the transmission of energy by electromagnetic waves or particulate matter (sub-atomic or nuclear). When radiation interacts with a medium, individual interactions have the ability to alter the electronic or nuclear states of the medium's constituent atoms and molecules. Some radiations possess, or have access to, enough energy to completely remove an electron from an atom (ionization). Such radiation, referred to as ionizing radiation, can be highly damaging to biological systems as it has the ability to disrupt the carefully maintained chemical and electronic balances within them.

\subsubsection{Radiation therapy}

Radiation therapy is the use of ionizing radiation to treat cancer and other diseases. Depending on a variety of case-specific factors, radiation treatments aim to cure the malignancy, prolong the life of the patient, and/or improve their quality of life. This is 
accomplished through careful treatment planning and sophisticated radiation delivery systems that allow radiation therapy professionals to maximize damage to the target area while simultaneously minimizing damage to healthy tissues.

\section{Brachytherapy}

Brachytherapy (from the Greek brachys, meaning short) involves the placement of sealed radioactive sources in close proximity to the malignancy, either temporarily or permanently. This comes with the benefit that dose, a quantity describing the amount of radiation absorbed by tissue (Section 3.3.2), can be highly localized to the actual disease site. However, brachytherapy procedures are often invasive and are only suitable for a subset of tumours [3].

\section{External beam radiation therapy}

In external beam radiation therapy (EBRT), the radiation source is placed outside of the patient and the radiation beam is shaped and directed at the disease. Radiation delivery systems that take advantage of the natural radioactivity of isotopes such as ${ }^{60} \mathrm{Co}$ have gone out of favour in the developed world for photon, electron, proton, and carbon ion beams generated using particle accelerators. Most notable of these is the linear accelerator (linac).

Linacs utilize microwave cavities to accelerate electrons to high energies (6-20 MeV). These electrons are then directed toward a heavy metal target using a series of magnets. The resulting collisions in the target produce photons of energy up to that of the electron beam through the bremsstrahlung effect (Section 2.1.2). A set of metallic collimators are placed in the path of the newly-formed photon beam in order to shape it before it exits the linac. The target can also be removed and the primary electron beam used instead. These beam delivery and shaping components are all housed within a rotating gantry and the patient is placed on a treatment couch in the path of the beam. A simplified linac schematic is shown in Fig. 1.1.

The linac is versatile and can be used in a number of modalities covering a large range of tumour types. For example, wide rectangular fields may be generated for wholebody irradiations. On the other extreme, the field shape may be changed dynamically as the gantry is rotated about the patient in order to deliver a highly conformal or intensity modulated radiation field to a target region. A more complete discussion of the myriad ways in which a linac may be used in the clinic can be found in textbooks such as Khan [5].

Beyond linacs, there are several other important classes of accelerators in clinical use. For example, orthovoltage radiotherapy units are often employed in the treatment of superficial tumours. These units are also based on the bremsstrahlung principle 


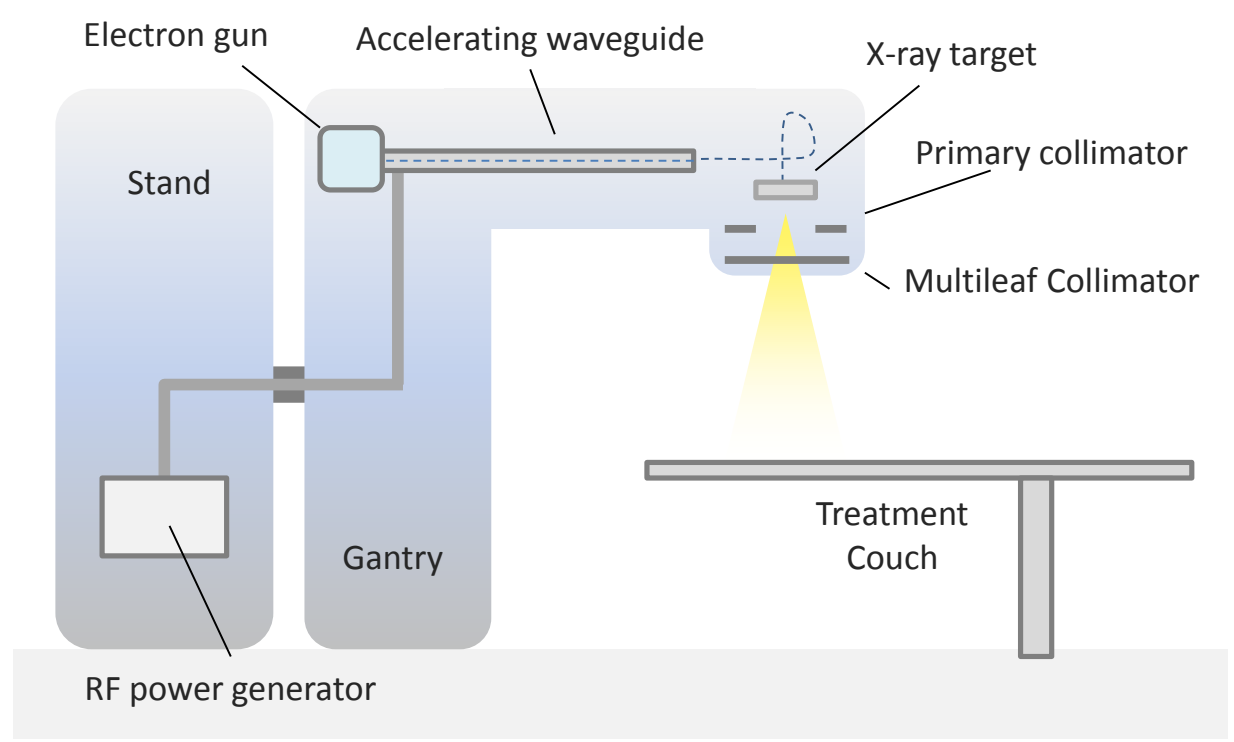

Figure 1.1: Simplified schematic of a medical linear accelerator. Reproduced with permission from Patrick [4].

but generate beams of much lower energies $(\sim 150-500 \mathrm{keV})$. So-called electronic brachytherapy units have also been developed that generate photon fields at energies similar to orthovoltage units. These highly portable devices are useful in irradiating tumour beds and treating skin lesions [6]. Gamma-knife machines are currently in clinical use for the treatment of brain tumours using pencil beams of ${ }^{60}$ Co radiation [5]. Therapies involving heavier particles such as protons and carbon ions have also become popular due to their beneficial dose profiles; however, there are still significant barriers preventing the widespread adoption of such techniques [7].

During EBRT treatments, radiation from the beam is scattered throughout the shielded treatment room (bunker) as it interacts with the patient, treatment couch, walls, and other objects. Leakage from the accelerator contributes additional photons and electrons to this out-of-field radiation and, in the case of high energy radiotherapy ( $\gtrsim 8 \mathrm{MeV}$ ), nuclear reactions fill the bunker with secondary neutrons. This out-of-field radiation deposits a whole-body, non-curative dose to the patient. Although too low to result in radiation burns or significant cell killing, the out-of-field dose still poses a risk for carcinogenesis and other long-term effects.

\subsubsection{Radiation carcinogenesis}

The vast majority of cancers result from environmental factors rather than inherited defects [8]. Fitting of the diversity of the disease itself, cancer may arise from any one of or a combination of factors including smoking, poor diet, inactive lifestyle, stress, pollutants, and, most relevant to this thesis, exposure to background and/or medical 
radiation. Generally speaking, subjecting healthy cells to stressful environments can lead to genetic instability. In the case of radiation carcinogenesis, this process occurs when interactions between ionizing radiation and the cell lead to the induction of mutations in cell stability genes [9]. These include the cell growth-promoting protooncogenes, their negative regulating counterparts, known as tumour suppressor genes, and deoxyribonucleic acid (DNA) stability genes involved in both the surveillance and repair of DNA damage.

Radiation carcinogenesis is a major concern in radiological protection. In radiobiological terms, radiation carcinogenesis is said to be a stochastic effect, meaning that cancer induction is a probabilistic process, there is no radiation dose threshold below which the induction of cancer is not possible, and the severity of the incurred cancer is independent of dose; only the likelihood of occurrence is dose-dependent. The so-called linear-no-threshold (LNT) principle states that even a small amount of radiation may induce sufficient mutations and genetic instability to initiate the process of carcinogenesis [10]. As a result, a core tenet of radiation safety is that radiation exposure should be kept As Low As Reasonably Achievable; the ALARA principle.

The complexity of the stochastic cancer-induction process makes radiation carcinogenesis difficult to quantify and predict. Much of our understanding of the risk comes from epidemiological data from Japanese atomic bomb (A-bomb) survivors, occupationally-exposed persons, and various accidental exposure incidents [11]. Extrapolating risks predicted by such data to situations experienced by the general population is not straightforward. For example, the A-bomb data are specific to high doses delivered to an ethnically homogeneous population and are primarily only useful for gamma radiation, as the doses from other particles such as neutrons were too low to reliably isolate their effects [12]. Moreover, stringent radiation protection protocols should preclude modern radiation workers from ever receiving such high doses as received by the Radium Girls (see The Radium Girls by Kate Moore [13] for a great account of this tragedy).

More recent retrospective studies have been able to address some of these shortcomings. The danger from low level exposures was demonstrated by Cardis et al. [14] for nuclear industry workers across 15 different countries, while several groups have demonstrated the risk of second cancers resulting from radiation therapy [9]. Extensive animal studies have also been performed [11] in controlled environments; however, there are still issues in applying the results to human populations, especially for neutrons [10].

\subsubsection{Numerical methods in radiation carcinogenesis studies}

Recent advancements in computational power have allowed for more fundamental studies of the relationship between radiation interactions and induced DNA damage [15]. 
This pursuit may help lead to quantitative, mechanistic predictions of radiation carcinogenesis. However, many limitations still exist, from our current description of radiochemical processes [16] to the exact causative link between mutagenesis and the induction of cancer [9]. Nevertheless, our understanding is sufficient to be able to compare the carcinogenic potential of various radiations on a relative, if not fully quantitative, basis.

It is well established that the presence, extent, and distribution of clustered lesionsmultiple DNA damage sites in close proximity - is linked to the mutagenic potential of a given radiation insult [17]. As the distribution of DNA damage sites arises from the spatial distribution of radiation interactions, referred to as the track structure (TS), any fundamental study of radiation carcinogenesis requires an accurate treatment of radiation transport through biological tissue. Multiple approaches exist to analyze numerically-generated track structures, including numerical microdosimetry (Ch. 4), clustering algorithms [18], and, more recently, multi-scale DNA damage simulations [19]. In the research presented in this thesis, a microdosimetric approach was taken.

Microdosimetry is based on the assumption that the spatial distribution of radiation interactions in biological tissue is predictive of the spatial distribution of the resulting chemical changes to DNA. As the complexity of DNA damage is correlated with mutagenic potential, a thorough study of radiation interactions on the scale of DNA is thus predictive of stochastic biological effects. Based on this assumption, we hypothesized that microdosimetry could be used to obtain a physical rationale for the energy dependence of neutron biological effectiveness for carcinogenesis. 


\section{Chapter 2}

\section{Radiation Physics}

As ionizing radiation moves through matter, it transfers its energy to the medium through a series of discrete interactions. Each of these interactions has an associated probability to occur, or cross section, that varies with factors such as particle type, energy, and the medium's electron density. Thus, radiation transport is a stochastic, rather than deterministic, process. On a macroscopic scale, however, random fluctuations largely balance out and one can reliably expect convergence towards mean values. In this chapter, a brief overview of the possible interactions between ionizing radiation and matter is given. The Monte Carlo (MC) method is then presented in the context of the modelling of radiation transport and the calculation of dosimetric quantities.

\subsection{Types of ionizing radiation and their interactions}

\subsubsection{Photons}

Photons are the quanta of electromagnetic radiation and are thus massless 'particles' that move at the speed of light $c$. Ionizing photons can be broken into two categories based on their sources of production. X-rays are produced extranuclearly, typically by bremsstrahlung or through the filling of inner shell electronic vacancies. On the other hand, gammas, or $\gamma$-rays, are generated as products of nuclear reactions or nuclear relaxation.

In therapeutic medical physics, which is the application of the principles and techniques of physics to the detection, containment, and eradication of cancer, the photons generated and encountered have energies below $\sim 20 \mathrm{MeV}$. Photons in this energy regime can undergo a large number of interactions with electrons and atomic nuclei, with the most important of these being Rayleigh scattering, Compton scattering, the photoelectric effect, pair and triplet production, and photonuclear absorption [20]. Depending on the photon energy $h \nu$ and the atomic number $Z$ of the absorbing medium, the relative probabilities of these interactions vary. For the soft tissue material $(Z \leq 8)$ 
and photon energies $(1 \mathrm{keV} \lesssim h \nu \lesssim 15 \mathrm{MeV})$ studied in this thesis, Compton scattering is the predominant interaction.

\section{Rayleigh scattering}

A photon incident on a tightly-bound electron may undergo Rayleigh, or coherent, scattering. In this interaction, the photon is scattered without transferring any of its energy to the electron, although a negligible fraction of the photon's energy is transferred to the atom as a whole in order to conserve momentum [21].

\section{Photoelectric effect}

In the photoelectric effect, the incident photon is fully absorbed by a tightly-bound orbital electron. The energy provided to the electron is enough to free it from the potential well of its atom and send it recoiling with kinetic energy:

$$
E_{K}=h \nu-E_{B}
$$

where $E_{B}$ is the binding energy of the electron.

\section{Compton scattering}

Compton, or incoherent, scattering involves the interaction of a photon with a looselybound electron. When this occurs, the photon may transfer some of its energy to the electron, causing it to recoil away from the atom. Depending on the energy of the photon and the angle of its deflection, the amount of energy transferred to the recoil electron varies. The energy $h \nu^{\prime}$ of the scattered photon is given by:

$$
h \nu^{\prime}=h \nu \cdot \frac{1}{1+\epsilon(1-\cos \theta)}
$$

where $\theta$ is the re-emission angle of the photon with respect to the direction of its incidence and $\epsilon=h \nu / m_{e} c^{2}$ is the energy of the incident photon normalized to the rest mass energy $\left(m_{e} c^{2}\right)$ of the electron. The recoiling electron exits the interaction site with kinetic energy $E_{K}$ (given by Equation 2.3) at an angle $\phi$, which is related to the photon scattering angle by Equation 2.4.

$$
\begin{gathered}
E_{K}=h \nu \cdot \frac{\epsilon(1-\cos \theta)}{1+\epsilon(1-\cos \theta)} \\
\cot \phi=(1+\epsilon) \cdot \tan (\theta / 2)
\end{gathered}
$$




\section{Pair and triplet production}

Pair production is a process in which a photon interacts with the electric field of a nucleus, resulting in the complete absorption of the photon and the creation of an electron-positron pair [21]. Due to the rest mass energies of the particles produced, this interaction has an energy threshold of slightly more than $2 m_{e} c^{2}(1.022 \mathrm{MeV})$, with nearly all of the excess energy being split between the recoiling electron and positron. A small amount of energy is also transferred to the nucleus in order to conserve momentum. A similar interaction may also take place between a photon and the electric field of an orbital electron. In this case, the threshold is $4 m_{e} c^{2}(2.044 \mathrm{MeV})$ and the kinetic energy of the orbital electron due to the momentum transfer is no longer negligible. As a result, the orbital electron is ejected in addition to the materialization of an electron-positron pair, leading to the term triplet production.

\section{Photoneutron production}

Photonuclear reactions, sometimes referred to as photodisintegration, are a set of interactions in which a photon interacts with a nucleus, thereby causing an alteration of the nuclear composition and the release of one or more nucleons (protons or neutrons) or nuclear fragments. As endothermic reactions, these processes all exhibit an energy threshold. The most probable of these reactions, especially in the context of photon radiation therapy, is the emission of a single neutron following absorption of the photon. For most nuclei, the threshold for this form of photoneutron production, denoted $(\gamma, n)$, is around $10 \mathrm{MeV}$ [21]. Although the cross section for the $(\gamma, n)$ reaction is negligible with respect to the overall attenuation of photons in absorbing media, the photoneutrons these interactions produce are nonetheless an important factor in radiation safety considerations. Indeed, they form the secondary neutron fields whose risk is under study in this thesis.

\section{Characteristic x-rays and the Auger effect}

When an electron is ejected from an atom, such as through one of the ionization events described above, or is excited to a higher energy state, the target atom may be left with an electron vacancy in an inner shell. Consequently, outer shell electrons will jump to fill them. In doing so, energy is released that is then radiated away. This radiation may take the form of a characteristic x-ray, so called because they have a discrete spectrum corresponding to the electronic structure of the source atom. Another possibility is that the energy is spent ejecting a different orbital electron in a process known as the Auger effect. 


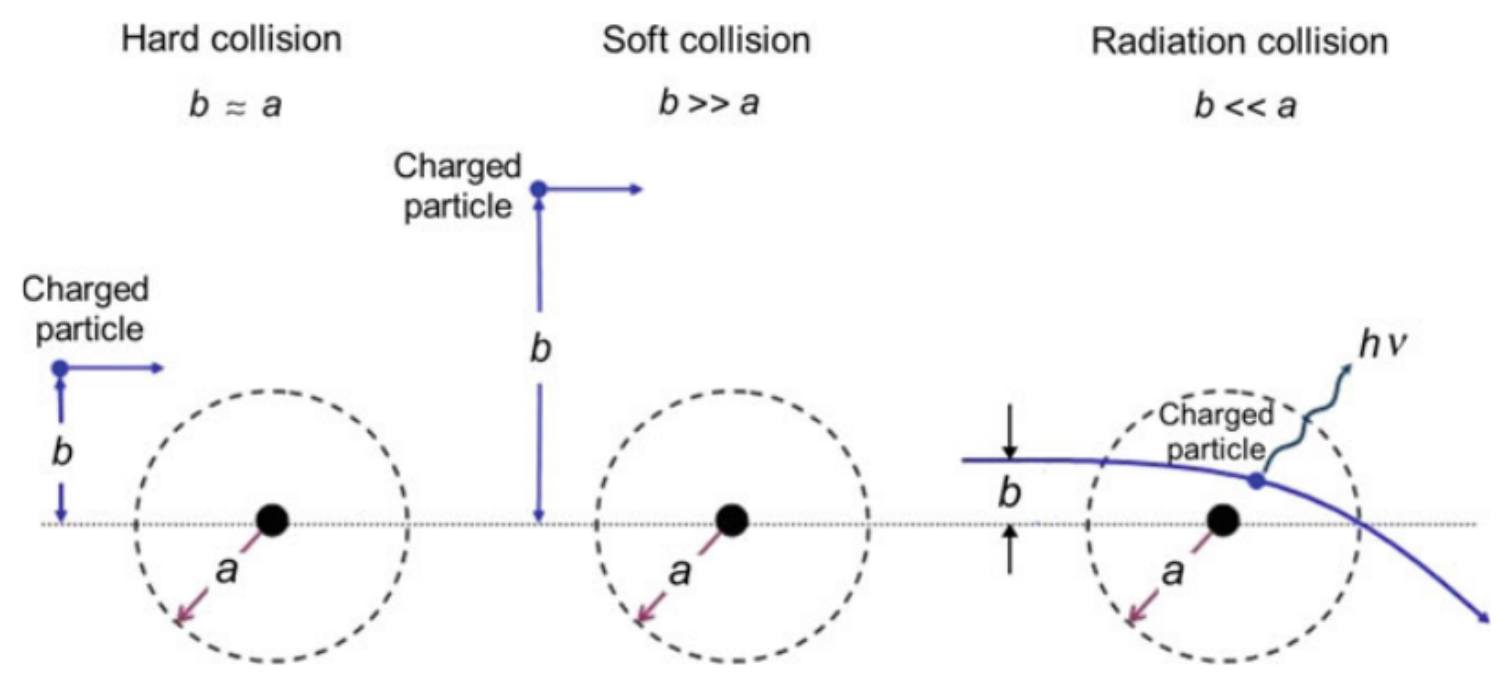

FIgURE 2.1: Classification of charged particle interactions with atomic constituents of absorbing media based on the impact parameter $b$ and the outer radius of the target atom $a$. Soft $(b \gg a)$ and hard $(b \approx a)$ collisions are particle-orbital electron interactions and radiation collisions $(b \ll a)$ are particle-nucleus collisions. Figure from Podgoršak (2016) [21].

\subsubsection{Charged particles}

Charged particles interact with absorbing media through Coulomb interactions with orbital electrons and atomic nuclei. Depending on the how the impact parameter $b$, which is the shortest distance between the particle's trajectory and the centre of the target atom, compares to the outer radius of the atom $a$, the Coulomb interaction is classed as either a soft, hard, or radiation collision. The former two are interactions with orbital electrons, while the latter is with the atom's nucleus. A schematic of these collisions is shown in Fig. 2.1.

\section{Particle-orbital electron interactions}

Interactions between incident charged particles and target orbital electrons result in the transfer of kinetic energy to the medium through atomic polarization, excitation, or ionization [21]. When the charged particle passes far from an atom with outer radius $a$ $(b \gg a)$, a soft collision may occur. In such cases, the incident particle interacts with the collection of bound electrons of the atom. For heavy charged particles $(Z>4)$, soft collisions make up the vast majority of all collisions; however, only a small amount of energy is transferred during each individual interaction. In contrast, hard collisions occur when the charged particle passes nearly tangentially to the outer radius $r$ of an atom $(b \approx a)$. The particle may then interact with a single orbital electron and transfer a relatively large amount of energy to it. Depending on the relative mass of the particle to that of the orbital electron, the maximum possible energy transfer varies. 
For example, positrons may transfer all of their kinetic energy in a single collision, while heavy charged particles may only transfer relatively small fractions. Soft and hard collisions are each responsible for about $50 \%$ of the total energy transferred from a heavy charged particle to the medium [21].

\section{Particle-nucleus interactions}

When a charged particle passes close to the nucleus of an atom $(b \ll a)$, it may interact with the Coulomb field of the nucleus itself and undergo what is known as a radiation collision. The majority of these reactions result in only slight angular deflections and are effectively elastic [21]. Due to the large mass of the nucleus, the amount of kinetic energy transfer required to conserve momentum is negligible compared to the energy of the incident particle. However, inelastic collisions may also occur, resulting in the deceleration of the particle. It is a fundamental law of nature that whenever a charged particle experiences any form of acceleration, part of its kinetic energy is radiated away in the form of photons. In the case of charged particles being slowed down in absorbing media, this process is referred to as bremsstrahlung production. At non-relativistic electron energies (electron velocity $v \ll c$ ), the bremsstrahlung (German for 'braking radiation'), radiation is emitted primarily perpendicular or near-perpendicular to the incident direction of the charged particle. As electron energy increases, the radiation is emitted in an increasingly forward direction and is confined to an increasingly narrow range of angles about the peak intensity, which is emitted at what is known as the characteristic angle. For a charged particle of atomic number $z$ and mass $m$ incident on an atom of atomic number $Z$, the overall bremsstrahlung intensity is proportional to $(z Z / m)^{2}$. The mass dependence demonstrates that heavy charged particles experience negligible bremsstrahlung compared to electrons and positrons.

\section{Stopping power and range}

Charged particles are often characterized by their stopping power, which is a term describing the rate of energy lost by the particle $(\mathrm{d} E)$ to an absorbing medium per unit path length $(\mathrm{d} x)$. Stopping power, typically given in units of $\mathrm{MeV} \mathrm{cm}^{-1}$, is energy, particle, and material dependent. It is also commonly divided by the material density $\rho$ to give the mass stopping power $S\left(\mathrm{MeV} \mathrm{cm}^{2} \mathrm{~g}^{-1}\right)$ :

$$
S=-\frac{1}{\rho} \frac{\mathrm{d} E}{\mathrm{~d} x}
$$

The total (mass) stopping power can be subdivided into multiple components: collision $\left(S_{\text {col }}\right)$ and radiative $\left(S_{\text {rad }}\right)$, describing the energy lost through particle-orbital electron and particle-nucleus interactions, respectively. Collision stopping power may be further 
divided into soft and hard components.

$$
S_{\mathrm{tot}}=S_{\mathrm{rad}}+S_{\mathrm{col}}
$$

For heavy charged particles, $S_{\text {rad }}$ is negligible and thus $S_{\text {col }}$ dominates at all energies. However, the radiative yield is much higher for light charged particles and increases linearly with $E_{K}$ and $Z$. At low energies, the collision component dominates, but at energies exceeding a $Z$-dependent critical energy the radiative component comes to dominate.

A related concept is the range $R$ of a charged particle in a given medium. Generally, range refers to the average path length the particle takes through a material before coming to rest. Heavy charged particle tracks are characterized by negligible deviations from their initial velocity vectors, small energy fluctuations, and little to no radiative losses. As such, they may well be seen as depositing their kinetic energy gradually and in a continuous manner. This is referred to as the continuous slowing-down approximation (CSDA) and the range it predicts can be calculated from the particle's stopping power:

$$
R_{\mathrm{CSDA}}=\int_{0}^{\left(E_{K}\right)_{0}} \frac{\mathrm{dE}_{\mathrm{K}}}{S_{\mathrm{tot}}\left(E_{K}\right)}
$$

where $\left(E_{K}\right)_{0}$ is the initial kinetic energy of the particle. $R_{\mathrm{CSDA}}$, given in $\mathrm{cm}^{2} \mathrm{~g}^{-1}$, is a very good approximation for the range of heavy charged particles. Conversely, light charged particles follow very tortuous trajectories and display far more statistical spread in the distance the particle will travel along its initial trajectory (straggling). Consequently, $R_{\mathrm{CSDA}}$ is a less useful value for electrons and positrons, although it does still provide a reasonable estimate. Several alternative values for electron range are in common use, such as the $50 \%$ range $R_{50}$ and the practical range $R_{p}$. These are based on measurements of dose as a function of depth in absorber for electron beams.

In medical physics, we are often concerned less with the total amount of energy transferred than with the amount transferred to some localized region of interest. For this reason, stopping power can be misleading, because hard collisions may provide enough energy to eject electrons ( $\delta$-rays) and send them far from the region of interest. To address this, the concept of restricted collision stopping power has been introduced. It is calculated in the nearly same way as collision stopping power (i.e. it ignores radiative loss) but it excludes energy transfers to $\delta$-rays greater than some upper threshold. 
TABLE 2.1: Neutron classification by kinetic energy. Reproduced from Andreo et al. (2017) [20].

Term used Kinetic energy range

Cold neutrons

$E_{\mathrm{K}} \ll 0.025 \mathrm{eV}$

Thermal neutrons $E_{\mathrm{K}}<0.5 \mathrm{eV} ;$ most probably: $E_{\mathrm{K}} \approx 0.025 \mathrm{eV}$

Epithermal neutrons

$1 \mathrm{eV}<E_{\mathrm{K}}<1 \mathrm{keV}$

Intermediate neutrons

$1 \mathrm{keV}<E_{\mathrm{K}}<50 \mathrm{keV}$

Fast neutrons

$50 \mathrm{keV}<E_{\mathrm{K}}<20 \mathrm{MeV}$

Relativistic neutrons

$E_{\mathrm{K}} \geq 20 \mathrm{MeV}$

\section{Linear energy transfer}

The quantity linear energy transfer (LET) $L_{\Delta}$ is the energy lost by a particle in traversing some distance through a medium. It is almost identical to the restricted collision stopping power, except the energy threshold $(\Delta)$ is set on the kinetic energy of the ejected $\delta$-ray rather than the total amount of energy transferred [22]. In other words, the binding energy of the $\delta$-ray is ignored. Like stopping power, LET varies with energy, particle type, and material. Consequently, as a particle slows down or crosses material boundaries, its LET will change. To correct for this, a particle's track can be broken into a number of equal distance intervals. The track average LET is then calculated by taking the mean of the distribution of interval $L_{\Delta}$ values [23].

\subsubsection{Neutrons}

Neutrons are electrically neutral baryons (odd number of valence quarks) with mass slightly greater than that of a proton. Like other neutral particles, they are indirectly ionizing; however, unlike photons, they interact primarily with atomic nuclei rather than with orbital electrons. Neutrons are typically found bound together with protons, thus forming atomic nuclei. Free neutrons, on the other hand, have a mean lifetime of only about 15 minutes [24], undergoing spontaneous decay into a proton, electron, and electron antineutrino.

\section{Classification}

Due to the marked energy dependence of neutron cross-sections, it is common practice to refer to neutrons based on the energy regime they occupy. They can be broken up into several categories as shown in Table 2.1: 


\section{Elastic scattering}

In direct elastic scattering, a neutron is deflected by the nuclear potential of the target nucleus without exciting it [25]. This leads to an energy transfer of:

$$
\Delta E_{K}=\left(E_{K}\right)_{i} \frac{4 m_{n} M}{\left(m_{n}+M\right)^{2}} \cos ^{2} \phi
$$

where $\left(E_{K}\right)_{i}$ and $m_{n}$ are the initial kinetic energy and mass of the neutron, respectively, $M$ is the mass of the target nucleus, and $\phi$ is the angle through which the target nucleus recoils relative to the initial trajectory of the neutron. Equation 2.8 is valid in the classical limit; at relativistic energies (beyond the scope of this thesis), the energy transfer fraction has a more complex energy and mass dependence. However, at all energies, the average energy transfer fraction decreases with increasing $M$. Direct elastic scattering can occur at all energies, resulting in a fairly constant cross-section for most materials across the energies studied in this thesis $(1 \mathrm{eV}-15 \mathrm{MeV})$.

Elastic scattering may also occur when the incident neutron forms a compound nucleus with the target [25]. The compound nucleus may relax to the ground state of the original nucleus through the re-emission of a single neutron, conserving kinetic energy between the two particles [26]. As compound nucleus formation only occurs within the neighbourhood of allowed nuclear transitions, the total elastic cross section exhibits sharp resonance spikes where compound elastic scattering can occur. This reaction is therefore sometimes referred to as resonance scattering.

\section{Inelastic scattering}

When the formation of a compound nucleus leaves it in an excited state, the compound nucleus may partially de-excite by emitting a neutron with a lower kinetic energy than the incident neutron. This leaves the nucleus in an excited state which will then relax to the ground state through the release of a gamma.

$$
\mathrm{n}+{ }_{\mathrm{Z}}^{\mathrm{A}} \mathrm{X} \longrightarrow{ }_{\mathrm{Z}}^{\mathrm{A}+1} \mathrm{X}^{*} \longrightarrow{ }_{\mathrm{Z}}^{\mathrm{A}} \mathrm{X}^{*}+\mathrm{n}^{\prime}+\gamma
$$

Such inelastic scattering, denoted $\mathrm{X}\left(\mathrm{n}, \mathrm{n}^{\prime}\right) \mathrm{X}^{*}$, is only possible above a material-dependent energy threshold. This is typically on the order of several MeV.

\section{Neutron capture}

In neutron capture, the compound nucleus fully de-excites through the release of a $\gamma$ or charged particle; denoted as $\mathrm{X}(\mathrm{n}, \gamma) \mathrm{X}$ or $\mathrm{X}(\mathrm{n}, \mathrm{x}) \mathrm{Y}$ for some particle $x$, respectively. Unlike inelastic scattering, neutron capture is possible for all neutron energies. Indeed, the capture cross-section increases towards lower energies in inverse proportion to the 
change in velocity. This $1 / v$ law is essentially a statement that the longer the neutron is within contact of the short-range nuclear force, the more likely it is to undergo a capture reaction [25]. Although most important at thermal energies relative to other interactions, capture cross-sections may also exhibit large resonance peaks at higher energies.

\section{Nuclear spallation}

At high energies ( $>100$ 's of MeVs), neutrons incident on heavy nuclei are capable of causing nuclear spallation. This process begins with an intra-nuclear cascade in which the energy transferred to the nucleus is spread through a series of nucleon-nucleon collisions [20]. Eventually, the nucleus reaches an equilibrated excited state that relaxes through the isotropic emission of large numbers of nucleons and nuclear fragments [20]. It is a relatively unimportant reaction in the context of photon radiatherapy safety considerations given the energies involved.

\section{Neutron-induced fission}

Neutron-induced fission involves the splitting of heavy nuclei into multiple smaller nuclei. It is accompanied by the release of a large amount of energy, some of which is used to emit neutrons. The fact that these freed neutrons can then go on to induce further fission and thus cause a chain reaction is exploited in the generation of nuclear power.

\subsection{The Monte Carlo method}

The MC method refers to a general class of numerical techniques for solving complex statistical problems. Through the use of (pseudo)random numbers, MC simulations seek to provide a reasonable estimate of expectation values by sampling from the relevant probability distributions. $\mathrm{MC}$ techniques are especially relevant today because current computational speed allows for their use in solving highly complex equations that would be far too difficult to approach analytically.

As radiation transport through matter is probabilistic in nature, MC is used extensively in radiation dosimetry applications. Given enough histories, macroscopic properties such as dose can be calculated with high accuracy. Furthermore, the nonanalytical nature of MC techniques means that they are very flexible with regards to irradiation conditions and geometry. The remainder of this section will describe the basics of MC in medical physics and the structure of the MC toolkit used in this thesis. 


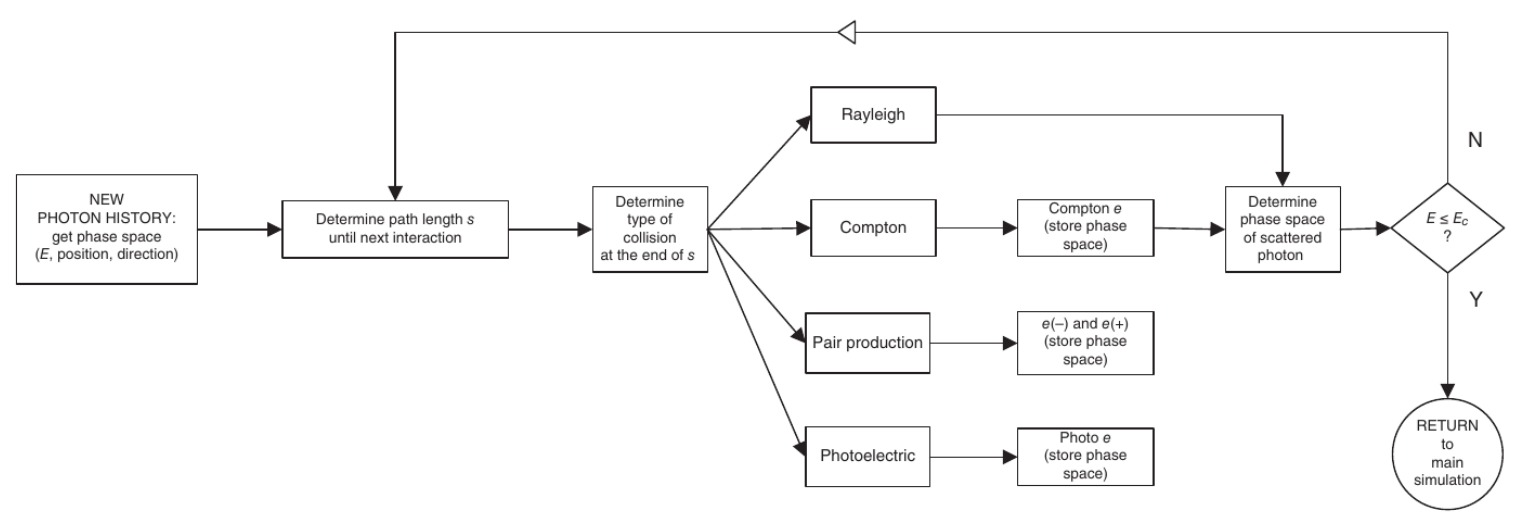

Figure 2.2: Flow diagram for the (analogue) transport of photons via the Monte Carlo method. Random numbers are used to determine the step length (distance until next interaction), the next interaction type, and the updated phase space of the photon following the interaction. The phase space of all generated secondaries are stored for future simulation. The photon is simulated until it is annihilated (e.g. undergoes a photoelectric interaction) or its energy falls below the cut-off $E_{c}$. Figure from Andreo et al. (2017) [20].

\subsubsection{Radiation transport with MC}

Radiation transport is governed by probabilistic cross sections and is thus well suited for MC. The discrete, stochastic nature of radiation interactions allows for their treatment as sudden, localized changes in particle energy and direction [20]. Between interaction points, the energy and direction of the radiation are assumed constant and thus radiation is said to follow well-defined zig-zag trajectories. The medium is assumed to be made up of randomly distributed scattering centres according to some predefined density.

Multiple random numbers are needed to transport particles between each individual interaction point. First, the expected distance until the next interaction, known as the mean free path (MFP) of the particle, is calculated according to the particle type and energy, material composition, and the scattering centre density. A random number is then used to sample a so-called step length according to the MFP. Subsequently, a second random number is used to select the interaction that occurs at the end of the step based on the relative magnitudes of the available cross sections. Finally, the energy change and angular deviation are sampled from the data for the chosen interaction. Any generated secondaries are queued for future simulation by storing their phase space (position and momentum) in a temporary location. This process continues until the initial particle meets an end condition. For example, if it leaves the region under study, its energy falls below a predefined cut-off value, or it is destroyed in some interaction. The queued particles are then simulated individually in the same way. An example of this process for photon transport is shown in Fig. 2.2.

MC studies tend to be time consuming and demanding on computational resources. 
A major bottleneck in the process is the transport of charged particles, especially at low energies. This can be addressed by decreasing the number of explicitly simulated interactions. Two commonly used methods exist to do so. The first has already been mentioned: an energy threshold can be chosen such that any time a charged particle falls below it, its energy can be assumed to be deposited locally and thus it no longer needs to be explicitly tracked (i.e. step-by-step with secondary production). The second is more complex and is based on the fact that charged particles undergo a large number of interactions that result in very small energy and direction changes (soft collisions). Using multiple scattering models and stopping power data, condensed-history $(\mathrm{CH})$ techniques approximate charged particle transport by combining the effects of several interactions into each step [27]. Hard collisions and bremsstrahlung production may be included separately to improve dosimetric resolution [28].

On macroscopic scales, CH techniques are the current clinical gold standard [29]. However, when one is interested in the nano- and micro-environment of cells, as in the research described in this thesis, the spatial accuracy of $\mathrm{CH}$ is insufficient. Instead, every interaction must be simulated discretely using event-by-event, or analogue, transport techniques. Purely analogue transport codes featuring low-energy physics models that allow cut-off thresholds $\lesssim 100 \mathrm{eV}$ are often referred to as track-structure (TS) codes.

The computation time of the MC simulations can also be improved through the use of a class of statistical methods known as variance reduction techniques. Especially useful in the study of rare events, these techniques aim to increase simulation efficiency without reducing the physical accuracy or introducing statistical bias [20]. The improvement in statistical accuracy associated with the implementation of variance reduction techniques would otherwise require a significant increase in computation time to achieve [30]. Some examples include particle splitting, Russian roulette, and correlated sampling [20]. Finally, modern MC codes often make use of parallel computer architectures through techniques such as multi-threading [31] or use of graphical processing units to maximize the resources available to a simulation at any given time.

\subsubsection{Geant4}

Geometry and tracking 4 (Geant4) [32-34] is an open-source Monte Carlo toolkit for radiation transport initially developed at CERN in Switzerland. Highly customizable and readily extensible, Geant4 provides users with a library of $\mathrm{C}++$ objects and classes to work with, enabling them to write their own object-oriented applications. Users are able to construct arbitrarily complex geometries composed of a large number of customizable elements and materials. Physical models and data sets allow for the simulation of the electromagnetic, hadronic, and optical processes of a great variety of particles over a large energy range ( $100 \mathrm{eV}$ to $1 \mathrm{PeV}$ depending on the particle). 
Classes governing particle generation, interaction, tracking, scoring, and visualization are provided and can be tuned to suit the needs of any given situation. Geant4 is used extensively in fields like high energy, nuclear, and medical physics.

\section{Radiation transport in Geant4}

In order to build an application using Geant4, a user needs to define the geometry, physics, and initial conditions of interest. Given this information, Geant4 is capable of a full simulation; however, it will not record or report anything. To extract relevant data, the user must assign sensitive detectors to volumes within the geometry and write a series of action classes.

A run in Geant4 is a collection of a specified number of events that share a common physical and geometric set-up; this information is immutable once a run has been initiated. Runs are controlled via the run manager. The user can write a run action class to add functionality to a run. For example, they may use this class to instantiate histogram objects at the beginning of the run and write the accumulated data to a file at the end of it. In multi-threaded mode, a multi-threaded run manager instantiates a specified number of worker threads, each with their own local run manager.

An event is the basic unit of simulation in Geant4. At the beginning of processing of an event object, the initial particles and vertices are used to generate primary tracks. These track objects are pushed to a stack (the temporary space described earlier) and popped one-by-one to be simulated. All secondary tracks are then pushed to the stack and the event ends when the stack is fully emptied. Event objects are handled by the event manager and their information can be accessed using an event action class. A stacking action class can also be written to alter the default behaviour of the stacking manager, such as adding a particle filter to restrict the tracks that are simulated.

In Geant4, tracks are snapshots; they only contain current physical information about the particle being simulated. They are updated by step objects. Step objects are made up of two step point objects, which contain information about the volume, material, and physical state of the particle either at the beginning or at end of the step. A stepping manager controls the processing of each step and a stepping action class can be used to access information about them. The stepping action class can also be used to control when to stop the simulation of a track. No information from the tracks will persist to the end of the event unless stored in trajectory objects.

To store simulation data, Geant4 provides an analysis manager for histograms and n-tuples (an that can hold an arbitrary number $n$ of different data elements) as well as an accumulable manager to easily update additive or multiplicative values ${ }^{1}$. These

\footnotetext{
${ }^{1}$ Use of these managers is not necessary; however, it is typically the best option in terms of code efficiency and readability.
} 
managers are instantiated in the run action class and can be accessed by all of the user action classes described above for a given run. Rather than accessing and writing to these objects each time an interaction of interest occurs, however, the sensitive detector method may be used to streamline this process. To do so, a user first decides on their regions of interest and assigns a sensitive detector to each one. When an interaction occurs in a sensitive detector, the user chooses what information to store from it (e.g. energy deposited, secondary particle type(s)). These hit objects are stored in a hit collection object associated with the sensitive detector and the current event. At the end of each event, the event action class allows the user to access the hit collections and record the data as appropriate. In this way, only the most necessary information is ever stored and data are only transferred to objects held by the run manager once per event. Finally, at the end of the run, the analysis and accumulable managers allow for the merging of data between events and threads and the writing of the combined results to an output file.

\section{Geant4-DNA}

Geant4-DNA [16, 35-37] is the radiobiological extension of Geant4. Included in the Geant4 public release, Geant4-DNA adheres to the nomenclature and structure of standard Geant4. It adds physical models that allow for the event-by-event tracking of electrons, protons, neutral hydrogen atoms, and various ionization states of the helium atom down to very low energies $(\sim 10-100 \mathrm{eV})$ in liquid water. Several heavier ions are also handled by analogue transport, although the lower limit for all of them is relatively high at $0.5 \mathrm{MeVu}^{-1}$. Beyond physical TS models, Geant4-DNA also handles what its maintainers refer to as the physico-chemical and chemical stages of radiation action. Between the end of the physical stage $(\sim 1 \mathrm{fs})$ and about 1 ps after the excitation or ionization interaction, a complex series of processes take place that ultimately result in the decay and dissociation, or radiolysis, of species altered during the physical stage. This physico-chemical stage is handled by probabilistic models that predict the population of reactive species generated by a given initial spatial distribution of excitations and ionizations [38]. From here, the chemical species are allowed to diffuse throughout the medium according to the memory-free Smoluchowski description of Brownian motion [39], which assumes thermal equilibrium and that particle diffusion does not affect the medium. A chemical kinetics data library is used to determine the probability of reaction between any two radiolytic products that pass close enough together or between these chemical species and the molecular constituents of the medium they are diffusing through [39]. The chemical stage covers up to the first microsecond following the radiation exposure. 



\section{Chapter 3}

\section{Radiobiology}

In contrast to other cytotoxic agents, ionizing radiation is not constrained by biological barriers such as the selectivity of cellular membrane channels; it may physically interact with any molecule in an organism. The effects arising from such interactions occur over a range of different time scales. While the physical processes described in Section 2.1 take place on the order of $10^{-15} \mathrm{~s}$, biological effects such as carcinogenesis may not be apparent for decades and heritable effects can potentially lie dormant for generations [9]. The first sections of this chapter will describe the initial stages of radiation insult and examine various biological endpoints from the perspective of radiation-induced physicochemical processes. The chapter will conclude by defining several important biological concepts and related quantities involved in the quantification and measurement of radiation.

\subsection{The genome}

The primary sensitive biological target correlated with the cancerous effects of radiation is deoxyribonucleic acid (DNA) [9]. Human DNA is a complex molecule that contains the entire genetic code and is thus responsible for the proper functioning of the cell, the transmission of the code to daughter cells, and, in the case of germ cells, the transmission of this code to offspring. Therefore, alterations to the DNA sequence, known as mutations, may affect the function of both the cell in which the biological target lies and potentially all of that cell's daughters. Before considering how radiation can cause mutagenesis and how the resulting mutations may result in cancer formation, it is useful to first consider the basic structure of DNA and the anatomy of genes.

\subsubsection{The DNA molecule}

The base monomer of DNA is the nucleotide. A nucleotide is made up of a deoxyribose sugar bonded to a phosphate and to one of four nucleic bases: adenine (A), thymine (T), cytosine $(\mathrm{C})$, and guanine $(\mathrm{G})$. A polymer of these acids, referred to as a DNA strand, is formed through strong phosphodiester bonds between the sugars. Two such strands 


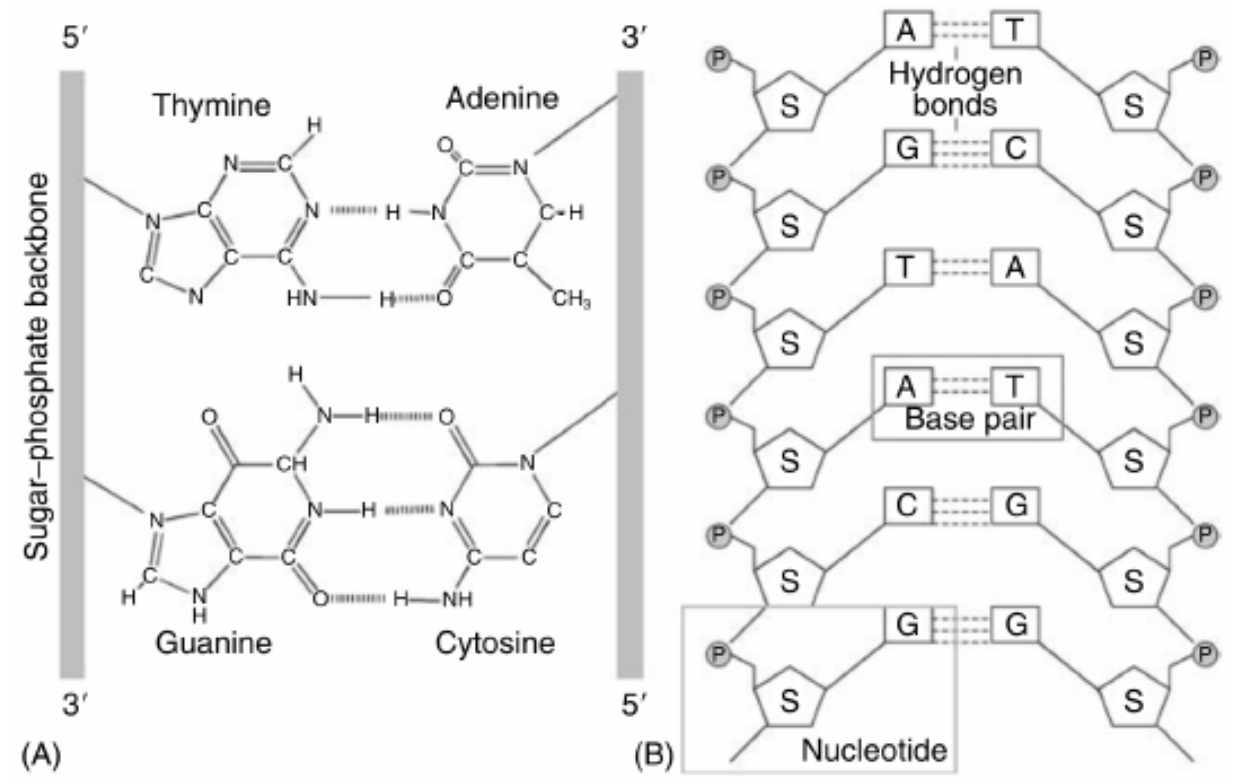

FiguRE 3.1: Individual DNA strands are composed of nucleotide monomers (a sugar attached to a nucleic base) connected by strong phosphate bonds between the sugars. Two strands are linked through a variety of weak bonds between bases. Notably, the selective hydrogen bonding between opposing nucleic bases (adenosine (A) with thymine (T) and cytosine $(\mathrm{C})$ with guanine $(\mathrm{G})$ ) provide both stability and a method for the replication of the genetic code. Strands are arranged anti-parallel, as denoted by the opposing 5' and 3' ends. (A) Molecular schematic showing the hydrogen bonding between complementary base pairs. (B) Simplified schematic of the DNA double helix indicating the relationship between the nucleotide monomers, the base pairs that connect them, and the hydrogen bonds that form the base pairs. Figure from Lehnert [40].

are aligned anti-parallel and interlinked by a variety of weak, non-covalent interactions. The most notable of these bonds are the hydrogen bonds that form between opposing bases. Crucially, this process is selective, as A can only pair with $\mathrm{T}$ and $\mathrm{C}$ only with $\mathrm{G}$ (Fig. 3.1). This selectivity allows for efficient replication of the genetic code, as one strand can always be used as a template for the formation of another complementary strand.

\subsubsection{Higher order structure}

The two DNA strands are twisted about each other into a double helix shape, thereby creating an interior hydrophobic environment. This separation of the bases from the surrounding molecules allows non-covalent bonds to form. The double helix is then wrapped about stabilizing and protective histone proteins in a structure called a nucleosome. A network of stabilizing proteins and forces then condense nucleosomes into chromatin fibres and fibres into chromatids. As humans are diploid organisms, 
each chromatid is paired with a sister chromatid (one from each parent) to form chromosomes.

\subsubsection{Codons and the gene}

To express a gene coded for by DNA, the exact sequence of base pairs is first transcribed into a (messenger) ribonucleic acid (RNA) molecule, which is a less stable polymer of molecules similar to DNA. RNA molecules then relay the sequence of base pairs to protein-generating ribosome complexes located outside of the nucleus. At these complexes, each set of 3 base pairs, called codons, are translated into proteins by matching them to specific amino acids. This process is known as the Central Dogma of molecular biology [41] and is the mechanism by which mutations can effect changes in cellular function. Interestingly, the number of amino acids is lower than the possible codon combinations, with some amino acids resulting from multiple distinct base pair sequences; this is known as the wobble effect.

Critical to the impact of mutagenesis, not all base pairs or codons actually code for proteins. Apart from regions of the genome that are completely non-coding, genes themselves are broken up into several unique sections (Fig. 3.2). These include regions both 'upstream' and 'downstream' of the coding region as well as the coding region itself. Codons in the coding region can be either coding or non-coding for a given protein, which allows for genes to code for multiple proteins. This is accomplished through a process known as splicing, by which certain subsets of the coding region are connected during post-processing of the transcribed RNA molecule. The upstream and downstream regions of the gene contain various important sequences responsible for binding molecules associated with the RNA transcription process (the promoter) and influencing the rate of expression of the gene. Due to the varied functions of DNA monomers within the genome, the range of effects mutations may cause is large and difficult to predict. Small mutations (a few base pairs) have the potential to completely alter the expression of all proteins associated with a gene, while the removal of a larger section of non-coding DNA ( $~ 98 \%$ of the human genome [42]) may have little phenotypic impact.

\subsection{The biological action of ionizing radiation}

\subsubsection{Direct vs. indirect action}

Due to its ability to excite, ionize, and generally alter atoms, ionizing radiation can disrupt the chemical stability of the DNA molecule and induce mutagenesis. The initial impact of radiation is often discussed in terms of strand breaks - the breaking 


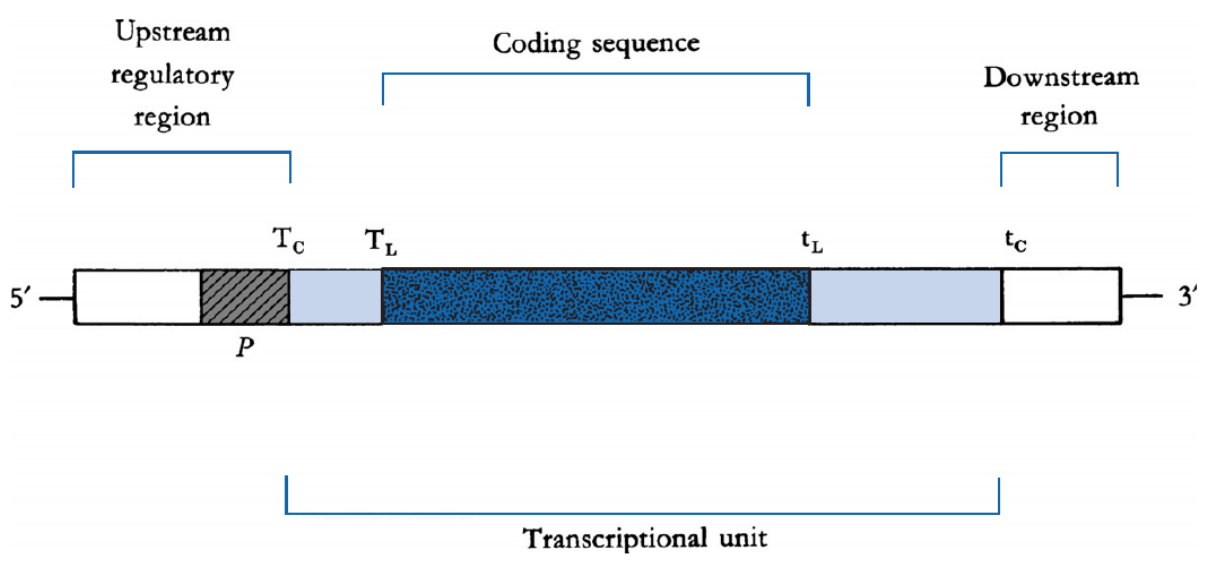

Figure 3.2: Simplified representation of gene anatomy. The regions upstream and downstream of a gene are primarily involved with regulating transcription while the upstream promoter provides a binding site for the transcription molecules. The transcription unit is region of DNA that is transcribed into RNA. $T_{C}$ is the transcription start site and $t_{C}$ is the transcription stop site. The portion of the gene that will eventually be translated into amino acids (subject to removal by splicing) occurs between the translation start $\left(T_{L}\right)$ and stop $\left(t_{L}\right)$ sites. Figure from Nicholl [43].

of bonds within the sugar-phosphate backbone. The means by which these breaks are caused are classified into two broad categories: direct and indirect action. In direct action, the incident radiation interacts with a component of the DNA molecule itself. Indirect action is a more complex process involving the creation of reactive radiolytic products [40] and is expanded upon below. An illustration of the two pathways is shown in Fig. 3.3.

Radiation incident on DNA can interact with surrounding molecules and lead to the formation of free radicals and other reactive products. Free radicals are chemical species that have an unpaired valence electron and are thus characterized by high chemical reactivity [9]. The most important of these radical-forming interactions is the induction of water radiolysis. When a water molecule is ionized or excited, the reaction products may proceed to react with other water molecules or dissociate, leading to the formation of radiolytic products including hydroxyl radicals $(\mathrm{OH} \cdot)$, hydrated electrons $\left(e_{\mathrm{aq}}\right)$, hydronium ions $\left(\mathrm{H}_{3} \mathrm{O}^{+}\right)$, and hydrogen atoms $(\mathrm{H} \cdot)$.

Indirect action occurs when these radiolysis products diffuse away from their sites of origin and undergo chemical reactions with DNA molecules. The distance that radiolytic products diffuse is unclear and how it should be modelled in Monte Carlo applications has been the subject of debate in the scientific community; however, it is generally accepted that the scale is on the order of nanometres (cf. [9], [44], [19]).

The predominance of one action or another depends on the type and energy of 


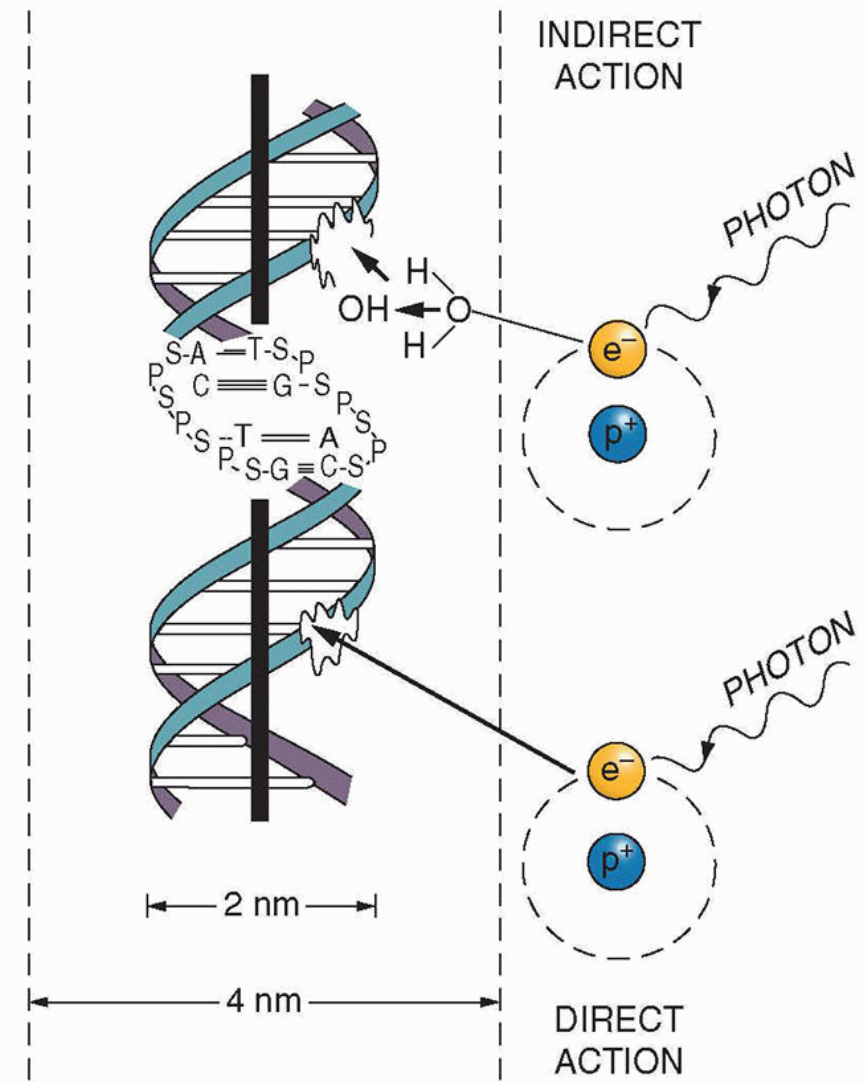

FIGURE 3.3: Direct vs. indirect action of ionizing radiation on DNA. In direct action, interactions occur between the incident radiation and a DNA strand. Indirect action involves the induction of water radiolysis and the chemical reactions of the radiolytic products with DNA. Figure from Hall and Giaccia [9].

the radiation. High LET radiations such as neutrons produce most of their damage through direct action, while for lower LET radiation indirect action is dominant. For example, approximately two thirds of strand breaks caused by x-rays are due to indirect action [45].

\subsubsection{Radiation-induced DNA damage and repair}

Through either direct or indirect action, ionizing radiation is capable of causing a variety of chemical changes to the DNA, including base or sugar damage, the removal of bases, and DNA-protein cross-links [11]. In the seconds and minutes following radiation-induced genetic damage, DNA repair pathways are activated in the cell [46]. The choice of pathway depends on the type and extent of damage as well as the stage of the cell cycle. Some types of damage are quick and simple to repair, while others are more time-consuming and susceptible to misrepair. It is only if and once the repair pathway has failed that the induced damage can lead to large-scale biological 
consequences. Thus, it is insufficient to only consider the initial yield of strand breaks when assessing biological damage.

Single strand breaks (SSB) are readily repaired because the undamaged complementary DNA strand can be used as a template [40]. When SSBs occur on opposing strands, the intervening bases are often enough to hold the helix together and allow straightforward repair of both breaks independently. However, when SSBs occur close together (within $\sim 10$ base pairs), the helix is split and more complex repair pathways are necessary. These breaks, known as double strand breaks (DSB), are more highly correlated with biological damage because they are more difficult to repair. Furthermore, DSBs are typically accompanied by extensive base damage and protein cross-links (covalent bonds between nucleotides and proteins that disrupt replication and transcription [47]) and are thus considered to be the primary lesions responsible for both mutagenesis and larger scale damage [11]. However, without information about their spatial distribution, DSB yield is still not a sufficient proxy for biological impact [17].

Clustered lesions (also known as locally multiply damaged sites, or LMDS) may contain one or more DSBs, SSBs, damaged bases or other forms of DNA damage within a short section of DNA [9]. Such lesions are associated with poorer repair probability than when the damage sites are more spaced out and are thus biologically significant features [17]. The inability to effectively repair clustered lesions can lead to a variety of endpoints associated with cell death and mutagenesis. Notably for carcinogenesis, stable chromosomal rearrangements stemming from the rejoining of a separated strand portion to the wrong break-point (translocation or deletion: Fig. 3.4) may result in the inactivation of tumour suppressor genes or the activation of proto-oncogenes [48]. The ability of single tracks of ionizing radiation to induce such complex lesions is part of the rationale behind the concept of the LNT model of radiological harm [44].

\subsection{Important quantities and concepts in dosimetry and radiation safety}

This section introduces some of the relevant quantities and concepts used to describe and measure radiation exposures.

\subsubsection{Physical quantities}

\section{Fluence and flux}

The particle fluence $\Phi$ is defined as the number of particles $\mathrm{d} N$ incident on a sphere of cross-sectional area $\mathrm{d} A$ centred at a point in space: 


\section{Translocation}
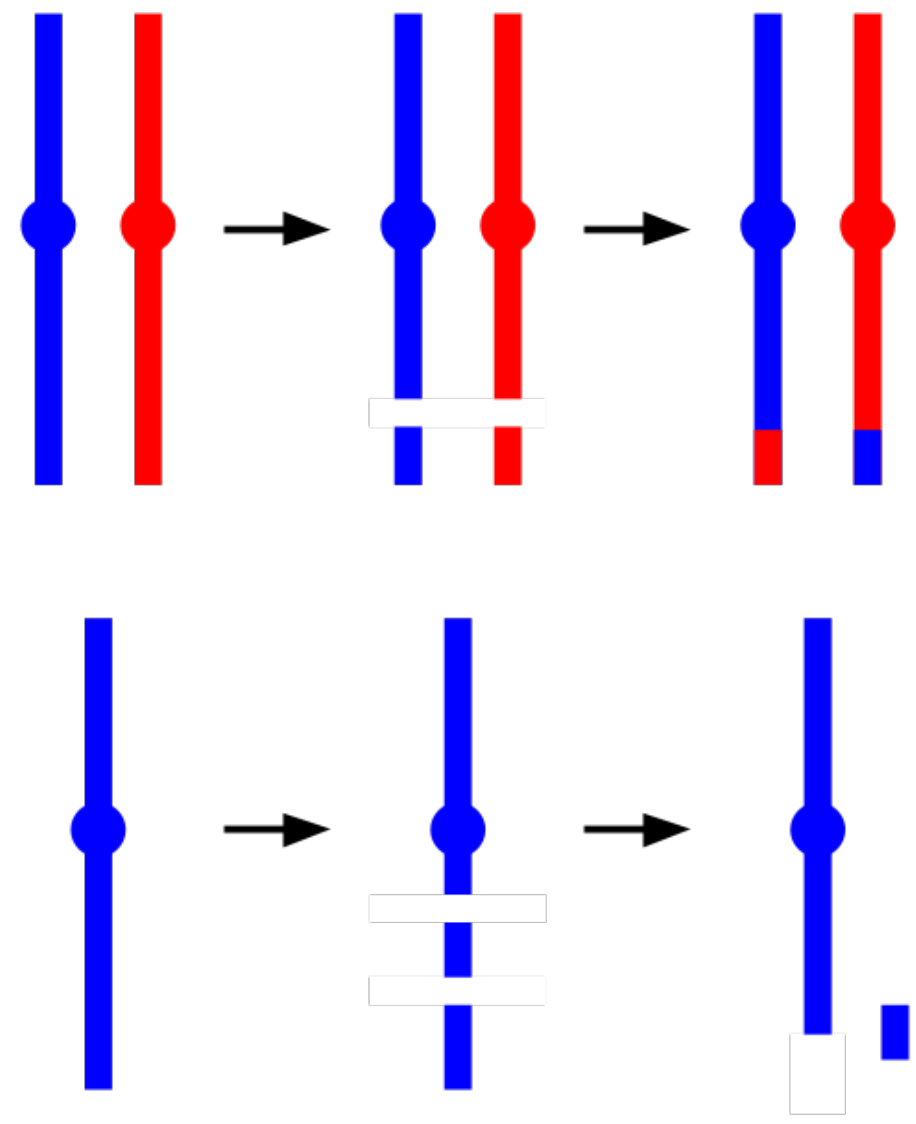

\section{Deletion}

FIGURE 3.4: Potentially carcinogenic chromosomal aberrations may result when radiation-induced clustered lesions are improperly repaired. Translocation occurs when a separated portion of one chromatid is mistakenly joined to a separate chromatid and vice versa. Deletion refers to the removal of a separated portion when rejoining the rest of the chromatid. 


$$
\Phi=\frac{\mathrm{d} N}{\mathrm{~d} A}
$$

The SI unit of fluence is $\mathrm{m}^{-2}$. A mathematically equivalent and more practical definition is based on the concept of path lengths $\mathrm{d} l$ taken by a particle through any arbitrary convex body [49]. For a volume $\mathrm{d} v$, this definition states that the average fluence is equal to the sum of all path lengths $\mathrm{d} s$ divided by the volume and is the standard definition used in Monte Carlo simulations [50]:

$$
\bar{\Phi}=\frac{\sum(\mathrm{d} s)}{\mathrm{d} v}
$$

Particle flux (density), or fluence rate, is defined to be the time derivative of particle fluence and carries units of $\mathrm{m}^{-2} \mathrm{~s}^{-1}[51]$ :

$$
\dot{\Phi}=\frac{\mathrm{d} \Phi}{\mathrm{d} t}
$$

The above definitions can be extended to account for particle energy, resulting in the quantities energy fluence $\Psi\left(\mathrm{J} \mathrm{m}^{-2}\right)$ and energy flux $\dot{\Psi}\left(\mathrm{J} \mathrm{m}^{-2} \mathrm{~s}^{-1}\right)$. For monoenergetic beams, the above quantities are simply multiplied by the energy of the beam. In most scenarios, however, particle beams are polyenergetic. Thus, the fluence spectrum $\Phi_{E}(E)$ must be used:

$$
\Phi_{E}(E)=\frac{\mathrm{d} \Phi}{\mathrm{d} E}(E)
$$

The total fluence is obtained by integrating over the fluence spectrum.

\section{Kerma}

The kinetic energy transferred from uncharged to charged particles in a volume is described by the quantity kerma, which is an acronym of "kinetic energy released per unit mass". It is expressed mathematically as:

$$
K=\frac{\mathrm{d} E_{\mathrm{tr}}}{\mathrm{d} m}
$$

where $\mathrm{d} E_{\mathrm{tr}}$ is the expectation value of the sum of the initial kinetic energies of all charged particles liberated by uncharged particles in a volume with mass $\mathrm{d} m$. This quantity is independent of whether the energy released is absorbed within the volume or not [20]. The unit of kerma is the gray (Gy), which is defined as the SI unit combination $\mathrm{J} \mathrm{kg}^{-1}$.

Kerma can be separated into electronic and radiative kerma. Electronic kerma $K_{\mathrm{el}}$ refers to the amount of kinetic energy dissipated relatively close to the interaction point. Radiative kerma $K_{\text {rad }}$ accounts for the rest. The mean fraction of kinetic energy 
that is converted back into uncharged radiation is called the radiative fraction $\bar{g}$ and can be used to relate electronic kerma with total kerma:

$$
K_{\mathrm{el}}=K(1-\bar{g})
$$

Kerma rate $\dot{K}$ is defined as the time derivative of kerma and is given by the units $\mathrm{Gy} \mathrm{s}^{-1}$.

\subsubsection{Dosimetric quantities and concepts}

\section{Absorbed dose}

The quantity absorbed dose $(D)$ is of significant importance in medical physics, as it concerns the amount of energy absorbed by a target. Technically, it is a point quantity defined as the energy imparted to an absorber material divided by its mass, in the limit that its volume approaches zero [20]. However, it is commonly referred to in the context of the average absorbed dose across a macroscopic volume [52]. Under this definition, the (average) absorbed dose is calculated as the amount of energy absorbed $\left(\Delta E_{\text {abs }}\right)$ in a volume of mass $\Delta m$ :

$$
D=\frac{\Delta E_{\mathrm{abs}}}{\Delta m}
$$

Throughout this thesis and in many medical physics texts, the average absorbed dose is referred to as either absorbed dose or simply dose. The unit of dose is the gray.

\section{Charged particle equilibrium}

Charged particle equilibrium (CPE) is said to exist in a volume $v$ (internal to a larger volume $V$ ) if the expected number of charged particles of a given type and energy that leave it is balanced by the expected number of the same type and energy that enter it (Fig. 3.5). For external sources of uncharged particles, CPE exists if the following conditions are met:

(i) the density and composition of the medium are homogeneous

(ii) the uncharged field is uniform throughout the medium (negligibly attenuated)

(iii) the minimum distance between the outer boundary of $v$ and the inner boundary of $V$ is greater than the maximum range of the secondary charged particles

When CPE exists in a volume, absorbed dose is equal to electronic kerma:

$$
D \stackrel{\mathrm{CPE}}{=} K_{e l}
$$




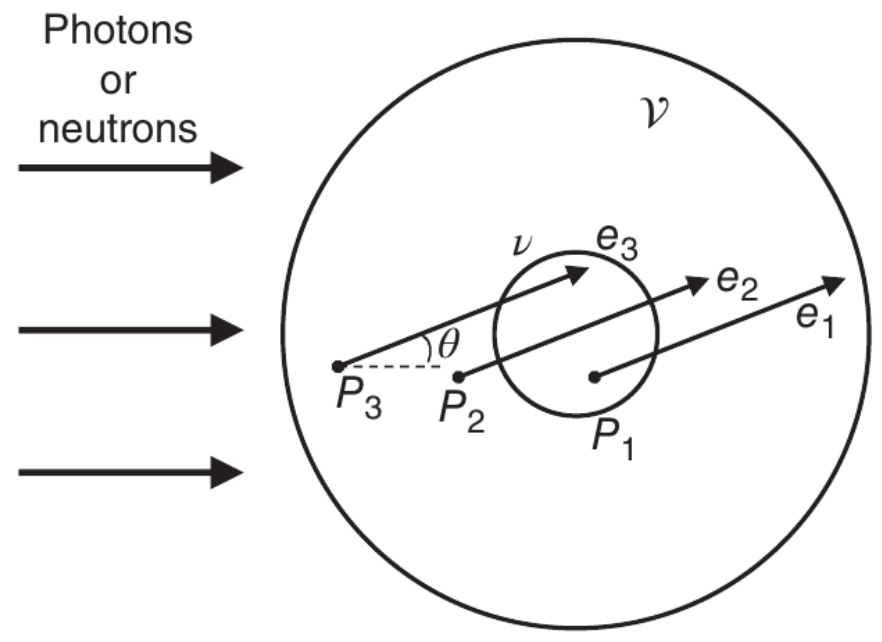

FIgURE 3.5: CPE exists for an external source of uncharged radiation if the volume $V$ is homogeneous, the radiation is negligibly attenuated across it, and the internal volume $v$ is separated from the boundary of $V$ by a distance greater than the maximum range of the secondary charged particles produced. Figure from Andreo et al. [20].

Thus, if the energy spectrum of the liberated secondary charged particles is known and their radiative losses are negligible, the dose can be calculated without knowing the full slowing down (energy fluence) spectrum of the charged particles. This is sometimes referred to as the local approximation and has been used extensively in neutron dosimetry calculations [12].

\section{RBE}

Relative biological effectiveness (RBE) is the ratio of the doses required for a test radiation to induce the same biological effect as a reference radiation:

$$
R B E=\frac{D_{X}}{D_{\text {test }}}
$$

where $D_{X}$ is the dose required by the reference radiation, typically $250 \mathrm{keV}$ x-rays or gamma rays emitted by a ${ }^{60} \mathrm{Co}$ source, and $D_{\text {test }}$ is the dose required by the test radiation to produce a specified biological effect. RBE varies with many factors, including the biological system and endpoint, the dose and dose rate, and the particle type and energy. For example, neutron RBE is believed to increase with decreasing dose and to be higher for carcinogenesis than for cell killing [53].

RBE tends to increase with increasing LET, regardless of endpoint, biological system, or dose. However, experimental observations of irradiated human cells have found that RBE for cell survival (i.e. the reduction of the cell population to the

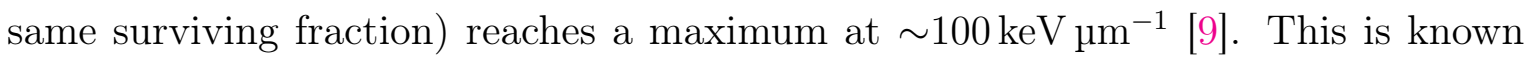


in radiobiology as the overkill effect and is attributed to 'wasted' dose, meaning that further increasing the energy density across a region (and therefore the dose) no longer increases the probability or severity of DNA damage and the cellular effect under study. Consequently, a greater dose has been delivered to achieve only the same result. These findings highlight the importance of considering the pattern of energy depositions produced by ionizing radiation when discussing biological effects.

\section{Equivalent dose}

The dependency of RBE on the type and energy of radiation is important to consider in radiation protection. To this end, the International Commission on Radiological Protection (ICRP) introduced the quantity equivalent dose $\left(H_{T}\right)$. It is defined as the weighted sum of doses to a particular tissue or organ $T$, where the amount of dose deposited by a given radiation type and energy $D_{T, R}$ is multiplied by an appropriate radiation weighting factor $w_{R}$ :

$$
H_{T}=\sum_{R} w_{R} D_{T, R}
$$

The radiation weighting factors are conservative estimates of the relative risk of inducing stochastic effects. The latest ICRP recommendations are published in ICRP Publication 103 [10] and are based upon large collections of radiobiological experiments, simulations, epidemiological data, and the ICRP's own judgement. For example, the weighting factors for photons and electrons are 1 for all energies, while those for neutrons vary between about 5 and 20 according to the continuous function shown in Fig. 3.6. The United States Nuclear Regulatory Commission (US NRC) has also recommended their own set of neutron weighting factors [54]. Differences between the two sets of recommended factors are discussed in the context of the results of this thesis in Ch. 7. The unit of equivalent dose is the sievert $(\mathrm{Sv})$, which is mathematically equivalent to the Gy.

\section{Effective dose}

The variations in the inherent radiosensitivity between tissues and organs should also be considered when assessing the biological impact of radiation. For this purpose, the ICRP recommends a set of tissue weighting factors $w_{T}$ to be applied to the calculated equivalent doses. The quantity effective dose $(E)$, also expressed in Sv, can then be calculated by summing over all exposed tissues and organs [10]:

$$
E=\sum_{T} w_{T} H_{T}
$$


ICRP Publication 103

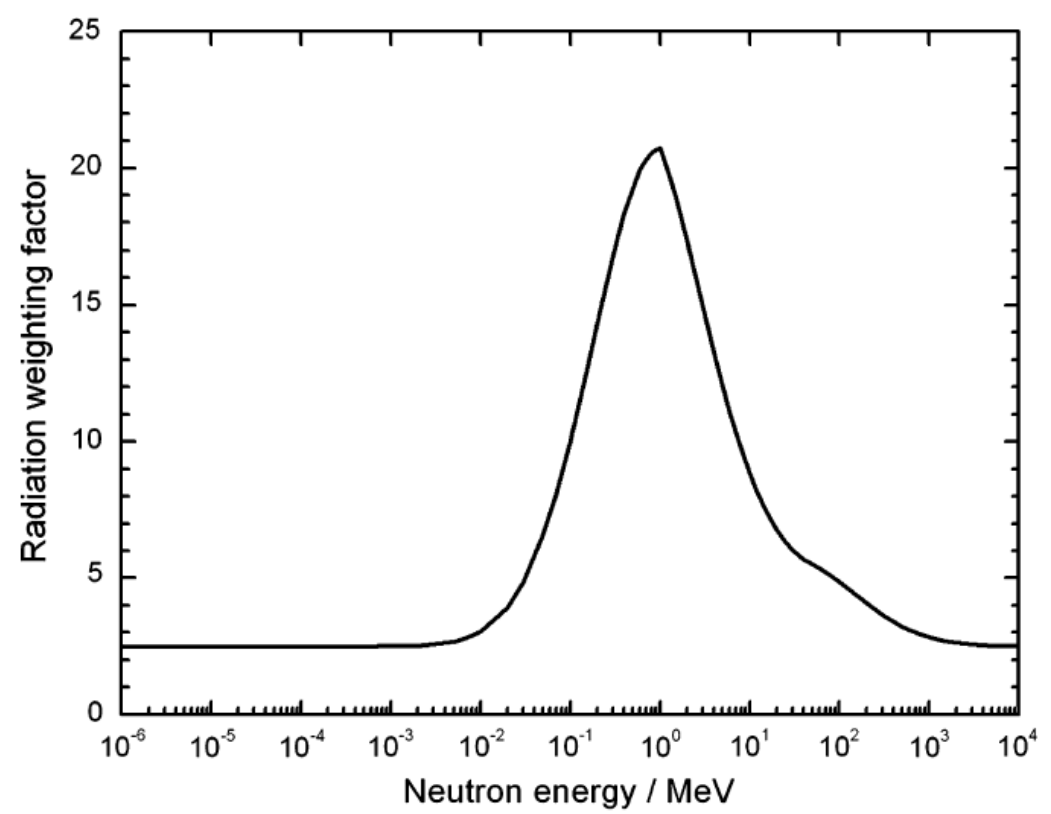

FIGURE 3.6: Neutron radiation weighting factors recommended by the ICRP [10]. 


\section{Chapter 4}

\section{Microdosimetry}

Macroscopic dosimetric quantities are insufficient to fully describe the potential of various types and energies of radiation to produce biological damage. The crux of this inability is that the transfer of energy from ionizing radiation to matter occurs through a discrete set of localized energy transfer interactions rather than through a more uniform energy dissipation process. As detailed in previous chapters, these interactions induce chemical changes through excitations and ionizations that disrupt important chemical balances, especially when clustered together. In addition, the spacing and size of the individual energy transfers are random, governed by cross sections and energy-angle distributions. Macroscopic quantities such as absorbed dose and LET average over these distributions, yet one cannot average over the biochemical damage arising from each interaction so readily. A full theoretical description of the biological consequences of irradiation must therefore begin with an accurate description of the stochastic energy deposition pattern. Microdosimetry, which is the detailed study of radiation interactions over microscopic volumes, provides an avenue for such characterization.

The following provides a brief introduction to microdosimetric theory, highlighting relevant quantities, concepts, and numerical calculation techniques. An overview of the application of microdosimetry to radiobiology with a focus on the stochastic effects of neutrons is then given to provide context for the work presented in the subsequent chapters.

\subsection{Important quantities and concepts}

Unit definitions are from ICRU Report 36 [55]. 


\subsubsection{The inchoate distribution and its intersection with sam- pling volumes}

In microdosimetry, interaction points at which energy is locally deposited to the medium are referred to as transfer points and their spatial distribution is known as the inchoate distribution. To calculate microdosimetric parameters, the inchoate distribution must be 'sampled' by considering its intersection with spherical or cylindrical volumes that represent biological targets. The choice of sampling volume size [52] and shape [56] has important consequences for the end result of the experiment or simulation. Direct experimental measurements are limited to detectors with sensitive volumes on the order of centimetres, although smaller volumes may be simulated through density corrections of measurements made in tissue-equivalent gas [57]. Numerical microdosimetry, on the other hand, is not quite so limited, although one must keep in mind that the smaller the simulation scale, the higher the uncertainty in the underlying models.

When considering the intersection of the inchoate distribution with an arbitrary sampling volume, the subset of transfer points in the overlapping region is referred to in terms of events. An event is the set of transfer points arising from a primary track and its associated secondary cascade that fall within a single sampling volume. When multiple tracks intersect the same sampling volume, it is said to be a multievent (Fig. 4.1). To further characterize the intersection, a specification of the size of the volume is given. Depending on the quantity of interest, the mass or mean chord length of the volume may be used. The chord length is the distance a straight line takes across a volume for some defined intersection. Under conditions of infinite, isotropic straight lines generated uniformly in space ( $\mu$-randomness), the mean chord length $\bar{\ell}$ for any convex body with volume $V$ and surface area $S$ may be calculated as $\bar{\ell}=4 V / S$ [49]. For a sphere then, $\bar{\ell}=4 r / 3$. Its use in place of the true path length taken by a particle, whose track may deviate significantly from rectilinearity, could be considered unnecessary in numerical simulations where the complete microscopic pattern is available. The choice of this definition is indeed rather arbitrary [58], but its use has been formalized in microdosimetric theory.

\subsubsection{Energy deposit and imparted energy}

The energy deposit $\epsilon_{i}$ is a stochastic quantity describing the energy deposited locally as a result of a single interaction $i$ :

$$
\epsilon_{i}=\epsilon_{\text {in }}-\epsilon_{\text {out }}+Q
$$




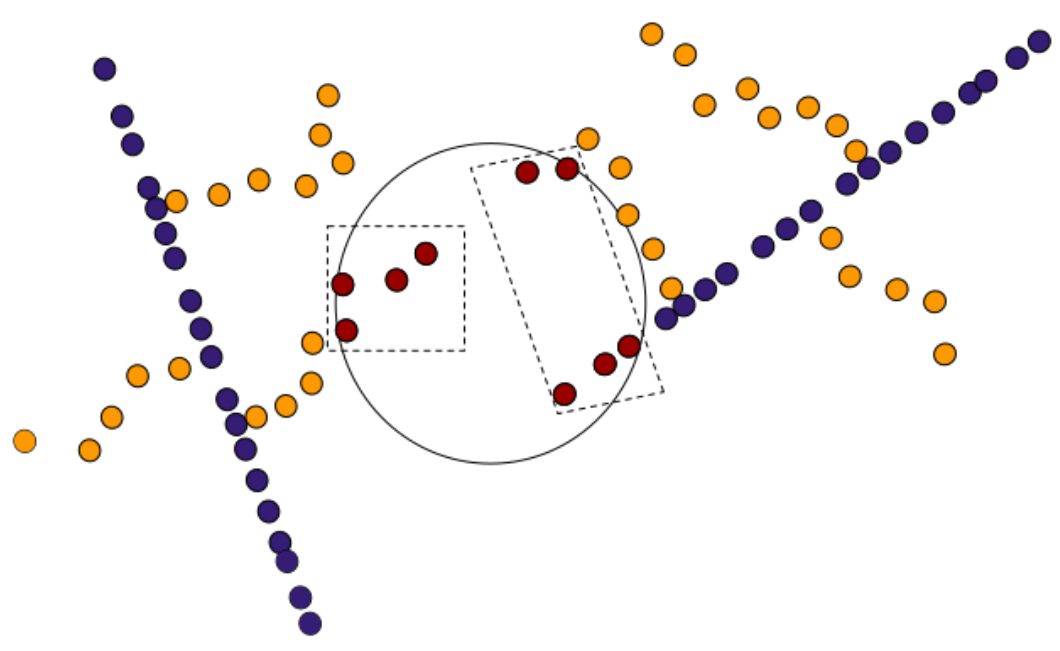

FIGURE 4.1: A 2-dimensional schematic depicting a multi-event situation in which two ion tracks intersect the same sampling volume (solid circle). Transfer points belonging to uncorrelated parent tracks are considered independent events. Primary ion transfer points are shown in purple and those of the delta electrons are shown in orange. The transfer points that fall within the sampling volume are red and the two events are demarcated by the dashed rectangles.

where $\mathrm{Q}$ is the change in rest mass of all particles involved $(>0=$ decrease, $<0=$ increase), $\epsilon_{i n}$ is the energy of the incident ionizing particle (excluding rest mass energy), and $\epsilon_{\text {out }}$ is the total energy (excluding rest mass) of all ionizing particles leaving the interaction [20]. Note that $\epsilon_{i}$ overestimates the amount of locally deposited energy as it does not allow for the possibility of non-ionizing particles leaving the interaction. Thus, $\epsilon_{i}$ actually represents the spatial region around an interaction within which the non-ionizing radiation is absorbed [21]. The sum of all energy deposits that occurred within a given volume is known as the energy imparted:

$$
\epsilon=\sum_{i} \epsilon_{i}
$$

It is a stochastic value and may be used in both single and multi-event situations [52]. In the case that only the contributions from a single event are considered in the summation, a subscript $s$ is often used to avoid confusion.

\subsubsection{Lineal energy}

Dividing the single-event imparted energy by the mean chord length of the sampling volume yields the lineal energy:

$$
y=\frac{\epsilon_{s}}{\bar{\ell}}
$$


Lineal energy is a stochastic measure of the spatial distribution of energy loss and is

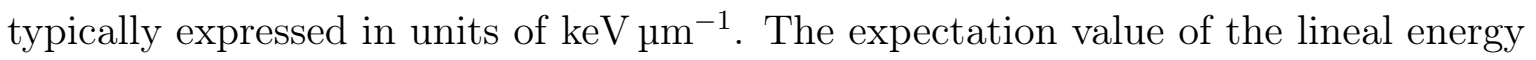
probability distribution, often referred to as the frequency-mean lineal energy, is calculated in the usual way:

$$
\bar{y}_{F}=\int_{0}^{\infty} y f(y) d y
$$

where $f(y)$ is the relative frequency distribution, i.e. the probability density function describing the probability that a measurement will yield a lineal energy value between $y$ and $y+d y$. The dose-mean lineal energy is the expectation value of the normalized size-weighted frequency distribution $d(y)=y f(y) / \bar{y}_{F}$ :

$$
\bar{y}_{D}=\int_{0}^{\infty} y d(y) d y
$$

$d(y)$ is typically referred to as the dose distribution because it represents the probability that $\epsilon$ is deposited (as dose) by lineal energy values between $y$ and $y+d y$ [59]. From the definition of $d(y)$, Equation 4.5 can be rewritten as:

$$
\bar{y}_{D}=\frac{1}{\bar{y}} \int_{0}^{\infty} y^{2} f(y) d y
$$

Thus, $\bar{y}_{D}$ can be calculated simply through knowledge of the frequency distribution by taking the ratio of its second moment to its first [58]. Note that as they are expectation values, $\bar{y}_{F}$ and $\bar{y}_{D}$ are non-stochastic.

Although $\bar{y}_{D}$ is defined for both uncharged and charged particles, the energy transfers it quantifies arise only from charged particle interactions. In order to calculate the $\bar{y}_{D}$ of an uncharged particle field, the effects of its various secondaries must be determined and combined [52]. To do so, one must weight the $\bar{y}_{D}$ of each species by its relative dose contribution and ensure that the energy dependence of the species' own dose distribution is taken into account. In other words, the $d(y)$ distribution must be subdivided by particle and energy. One approach to this is to calculate the dose differential in energy for each particle and combine this information with the dose-mean lineal energy for each energy that appears in the distribution [52]:

$$
\bar{y}_{D}=\int_{0}^{\infty} y d(y) d y=\sum_{i} \sum_{j} d_{i, j} \bar{y}_{D, i, j}
$$

where $d_{i, j}$ is the dose fraction due to species $i$ with energy $j$ and $\bar{y}_{D, i, j}$ is its corresponding dose-mean lineal energy. Note that:

$$
\sum_{i} \sum_{j} d_{i, j}=1
$$


Equation 4.7 has been used to calculate total $\bar{y}_{D}$ by using published data for $\bar{y}_{D, i, j}[60]$ and by explicit calculation [61, 62]. An alternative approach is to calculate the $\bar{y}_{D}$ of each secondary charged-particle spectrum directly. In this method, the spectra are gathered under conditions where the local approximation holds $\left(D=K_{e l}\right)$. Under these conditions, the normalized spectra are representative of the energy differential of the species' dose fractions. Consequently, a population of tracks generated by randomly sampling from the normalized spectra inherently accounts for the species-specific energy dependence. Taking the dose average of such a population gives the dose-mean lineal energy of the spectrum, thereby reducing Equation 4.7 to:

$$
\bar{y}_{D}=\sum_{i} d_{i} \bar{y}_{D, i}
$$

In other words, the $\sum_{j} d_{i, j}$ was performed implicitly, leaving one only to sum over $i$. Throughout the rest of this thesis, $d_{i}\left(\sum_{i} d_{i}=1\right)$ will be referred to as the relative dose contribution of species $i$. This approach has been applied to the study of mono-energetic neutrons [63] as well as to the spectra of various brachytherapy sources [64].

It should be noted that LET is a largely analogous concept to lineal energy that has been widely studied, measured, and used for radiation quality specification. Although it is a non-stochastic value, neither are the averages of the lineal energy distributions that are typically used to represent them. Indeed, under certain circumstances, numerical equivalency can be found between LET and $\bar{y}_{D}[52]$. It is therefore important to note that there are some conceptual differences between the two that necessitate the use of lineal energy on a microscopic scale. LET represents the average energy lost by the primary particle as it traverses a medium. As such, it contains no information on the radial spread of the energy lost, has no relation to microscopic sensitive volumes, and cannot account for the energy deposition profile of secondary species. Restricted LET may be used on smaller scales to correct for the transport of energy by high energy delta rays, but it cannot be measured [65] and contains no information about the actual track-structure of the particle under study. Therefore, even though $\bar{y}_{F}$ and $\bar{y}_{D}$ are non-stochastic values that sacrifice information on the exact shape of the true stochastic distribution, they are more useful for characterizing radiation quality in microscopic volumes than LET.

\subsubsection{Specific energy}

Dividing the energy imparted by the mass of the volume yields the stochastic value specific energy. 


$$
z=\frac{\epsilon}{m}
$$

As with lineal energy, frequency- and dose-mean values may be defined:

$$
\begin{aligned}
& \bar{z}_{F}=\int_{0}^{\infty} z f(z) d z \\
& \bar{z}_{D}=\int_{0}^{\infty} z d(z) d z
\end{aligned}
$$

Equation 4.11 can also be rewritten in terms of the average energy imparted:

$$
\bar{z}=\int_{0}^{\infty} z f(z) d z=\int_{0}^{\infty} \frac{\epsilon f(\epsilon) d \epsilon}{m}=\frac{\bar{\epsilon}}{m}
$$

For microscopic volumes, $\bar{z}_{F}$ is equivalent to the (average) absorbed dose of Equation 3.7. While specific energy is defined for multi-events, a strictly single event specific energy may also be used. In such cases, a subscript $s$ is added to avoid confusion. The information contained in $\bar{z}_{F, s}$ (single event frequency-mean specific energy) and $\bar{z}_{D, s}$ (single event dose-mean specific energy) is virtually the same as that contained in $\bar{y}_{F}$ and $\bar{y}_{D}$, respectively, and the choice of which to report is largely arbitrary.

\subsection{Track sampling}

\subsubsection{General considerations}

In numerical microdosimetry, the complete inchoate distribution is available for study with any number and orientation of sampling volumes. Many sampling algorithms have been developed to extract as much information as possible from each generated inchoate distribution. The simplest approach is to first define static sampling volumes, then randomly generate particle tracks and record all interactions in the sampling regions; analogous to how an ideal physical detector operates. A straightforward improvement to this is to invert the situation by first generating the tracks and then randomly overlaying volumes according to a uniform spatial distribution. Both approaches can accommodate single and multi-events. However, tracks are not guaranteed to intersect the sampling volumes and, as a result, such techniques are computationally inefficient. To address this data-wasting, one can instead bias the sampling by only placing volumes within the associated volume of a track [66].

The associated volume of a track is the region around it in which the sampling efficiency is 1 [67]. In other words, any sampling volume centred within the associated volume is guaranteed to contain at least one transfer point, while any volume centred 


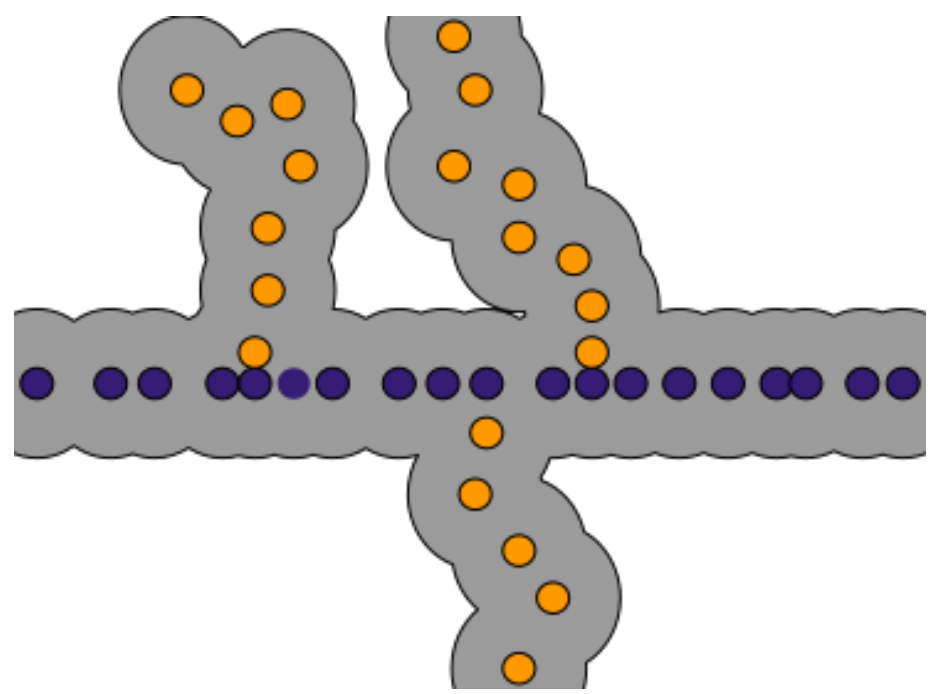

Figure 4.2: The associated volume of a track is the union of spheres of a chosen sampling radius centred on each transfer point. It provides a measure of the size of a track and a means with which to increase the efficiency of numerical algorithms.

outside of the associated volume will not contain any [68]. In the case of spherical sampling, depicted in Fig. 4.2, the associated volume is constructed by centring a sphere of the chosen sampling radius (the associated sphere or region) on each transfer point and taking their union [69].

Weighted sampling techniques can be used to ensure points fall within the associated volume without introducing statistical biases [58]. To do so, one first selects a transfer point at random and then selects a position within the associated region at which to centre a sampling volume [68]. Continuing with the spherical example, one would define a radial offset $d$ less than the sampling radius $r$ and apply it along a random direction to choose the centre position. One may then calculate $\epsilon$ by summing over all energy deposits that occurred within a distance $r$ of the chosen position and subsequently determine the $z$ and/or $y$ for the event. This process is depicted in Fig. 4.3. However, this technique is biased towards areas that are densely populated with transfer points. To correct for this, the calculated $\epsilon$ for a given sampling volume must be weighted by the inverse of the number of transfer points that fell within that volume [67].

\subsubsection{The weighted track-sampling algorithm}

A weighted sampling method was implemented directly into the TS simulation described in this thesis. An advantage of considering single event quantities such as lineal energy is that tracks can be processed individually. Thus, both track generation and analysis were multi-threaded in this work, which significantly reduced the amount of data to be stored or accessed at a given time. However, treating tracks independently exacerbates 


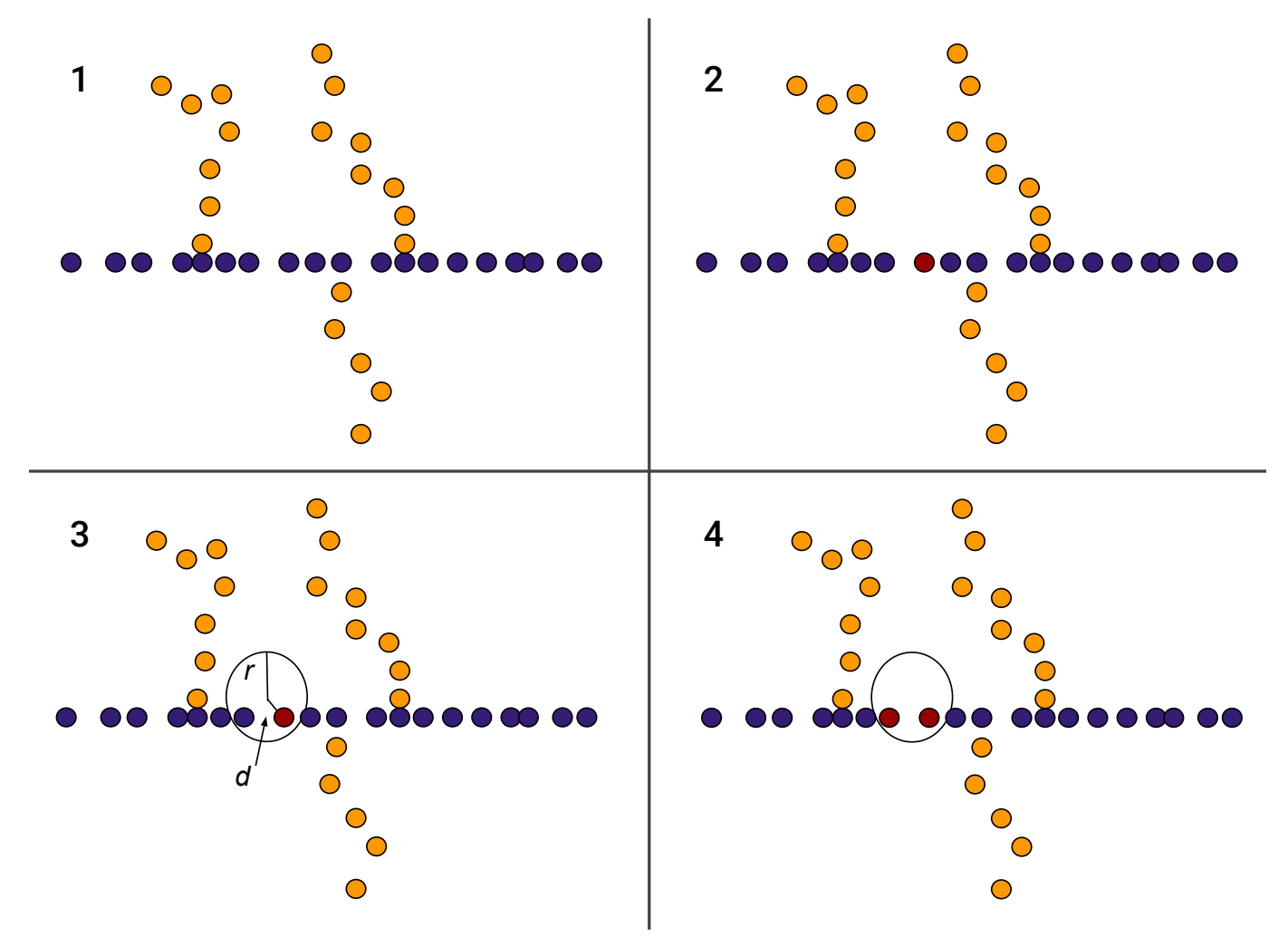

Figure 4.3: The weighted sampling method consists of the following steps: (1) track generation (transfer points of primary ions in purple and of delta electrons in orange), (2) the random choice of a transfer point (highlighted in red), (3) the placement of a sampling volume at a distance $d<r$ from the point, and (4) the summation of all energy deposits within this volume. 
a subtle issue with weighted sampling. Unless the entire population is considered when choosing a transfer point about which to place a sampling volume, then the relative size of the tracks cannot be taken into account. When sampling uniformly across all tracks simultaneously, larger tracks are more likely to be intersected by sampling volumes than smaller tracks. This thus implies a potential bias in the weighted sampling approach. Track size variation may not be significant or, more importantly, may not introduce systematic errors when considering monoenergetic particles. However, when polyenergetic spectra are considered as in the method discussed above, the lineal energy of shorter tracks may deviate significantly from those of larger tracks because the initial particle energy can vary by several orders of magnitude. The strong dependence of lineal energy on particle energy will be revisited in Chapters. 6 and 7. Consequently, it is important to ensure that all tracks are not treated equivalently. To this end, note that the associated volume of a given track is directly related to its probability of intersecting a sampling volume placed according to a uniform (but restricted to non-zero events) distribution. Famulari et al. [56] thus introduced an associated volume weight, in which the results from each track are multiplied by their associated volume and normalized by the sum of all associated volumes in the population. This approach requires a method for calculating the associated volume of arbitrarily sized and shaped tracks. A general solution to this problem is not attempted or presented here; it is a complex geometrical problem that was beyond the scope of this thesis. Instead, an approximate calculation based on a discretization of the sampling spheres into sub-voxels has been implemented. The details of this calculation are given in Ch. 5 . Throughout this thesis, the term weighted track-sampling algorithm refers to this version of the weighted sampling method.

\subsection{Microdosimetry in radiobiology}

The application of microdosimetry to radiobiology, radiation protection, and radiation therapy is well established. For example, lineal energy has been successfully used to predict the clinical effectiveness of neutron beams [70-72], proton beams [73], and carbon ion beams [74]. It has also been linked to such endpoints as chromosome damage in human cells [75] and cell survival in early responding tissues [23, 76] and has been used to show agreement between Japanese A-bomb data and the carcinogenic risk of occupationally exposed miners [77]. Indeed, microdosimetry has even been recommended for investigating situations in which the biological effectiveness is not well characterized [65].

Much of the motivation for the use of microdosimetry as a radiobiological tool rests on the theory of dual radiation action (TDRA) [78, 79], which provides a mathematical 
link between microdosimetric distributions and dose-effect relations such as RBE for cell survival. More specifically, the TDRA characterises the correlation between track structure and biological effect by considering the interplay of sub-lesions which, on their own, do not cause lasting damage. Variations in microdosimetric parameters can then be seen as adjustments in the range over which individual sub-lesions are able to interact and together cause the biological effect. Furthermore, the sub-lesion interactions can be subdivided into those that occur between sub-lesions resulting from the same track (intra-track, single event) and those arising from different tracks (inter-track, multi-event). In the case of low dose and low dose-rate environments, the assumption can be made that sensitive sites (e.g. cell nuclei) will only be crossed by single particle tracks and thus inter-track effects can be ignored. Under such conditions, the TDRA predicts that single-event microdosimetric distributions can be used to approximate RBE. Wuu et al. [69] utilised this formalism in their prediction of the RBE of low dose-rate brachytherapy sources, stating that:

$$
R B E_{\text {isotope }}=\frac{\bar{y}_{D, \text { isotope }}}{\bar{y}_{D, \mathrm{Co}}}
$$

where isotope refers to the radioactive source under study and ${ }^{60} \mathrm{Co}$ was the chosen reference radiation.

For neutrons, the 'low dose' assumption actually holds to very high neutron doses [78] and is even used in clinical situations wherein the RBE for late toxicities is assessed by lineal energy for fast neutron therapy beams [80]. In this thesis, the assumption was thus made that the neutron environment experienced by patients undergoing high-energy photon EBRT meets the 'low dose environment' criteria. The biological consequences of such neutron exposures therefore arise from the single event action of radiation and we hypothesize that the $\mathrm{RBE}$ for carcinogenesis can be investigated by comparing the $\bar{y}_{D}$ of mono-energetic neutrons to that of a reference radiation.

A similar approach was recently taken by the ANDANTE project [81], a multidisciplinary research collaboration who investigated the risk of neutron carcinogenesis through radiobiological experiments [82], numerical studies [63], and epidemiological models [83]. Building on previous work done by Satoh et al. [12] on the depth and energy dependence of secondary charged-particle dose contributions, Baiocco et al. [63] studied the depth dependence of single event microdosimetric distributions for external, mono-energetic neutron sources. Rather than explicitly generate tracks for their microdosimetric distributions, Baiocco et al. [63] utilized the microdosimetric function of the particle and heavy ion transport code system (PHITS) [84] MC toolkit. This function is able to calculate lineal energy for a large number of particles over broad energy ranges based on extrapolations from a track structure library [85] generated 
using the track structure of electrons in liquid water (TRACEL) [86] MC code. Thus, they could account for the heavy recoil nuclei. This necessitated the use of a modified version of $\bar{y}_{D}$ termed saturation-corrected dose-mean lineal energy:

$$
y^{*}=\frac{y_{0}^{2} \int_{0}^{\infty}\left\{1-\exp \left[-\left(y / y_{0}\right)^{2}\right]\right\} f(y) d y}{\int_{0}^{\infty} y f(y) d y}
$$

where $y_{0}$ is the so-called saturation parameter that is used to reduce the weight of high lineal energy values ( $\left.\gtrsim 100 \mathrm{keV} \mathrm{\mu m}^{-1}\right)$ in order to account for the overkill effect [87]. Baiocco et al. [63] used $y *$ as a proxy for neutron RBE by dividing the values for the neutron fields by those of $220 \mathrm{kV}$ x-rays and compared their results to the ICRP and US NRC weighting factors.

The Baiocco et al. [63] study served as a motivation for the research presented in this thesis. Due to the explicit generation and sampling of tracks reported here, however, a full comparison between end results cannot be made because the lack of low-energy heavy ion transport in Geant4-DNA precludes studies of neutron energies above $\sim 14 \mathrm{MeV}$ [88] (as was done in Baiocco et al. [63]). Rather, their simulation set-up serves as the basis for the methods presented in Chapter 5, while their search for a physical explanation for trends observed in published radiation weighting factors informs the direction of Chapter 7. 



\section{Chapter 5}

\section{Methods}

\subsection{Overview of the microdosimetric simulation pipeline}

The research described in this thesis focused on the development of a microdosimetric simulation pipeline and its application to the study of neutron RBE for stochastic effects. As this investigation was performed in the context of the neutron field experienced by a patient undergoing high energy radiotherapy, a single event proxy was chosen to represent these low neutron dose environments based on the TDRA. Namely, neutron RBE was approximated as the ratio of $\bar{y}_{D}$ values for mono-energetic neutrons to those for $250 \mathrm{keV}$ x-rays:

$$
\operatorname{RBE}\left(E_{K}\right)=\frac{\bar{y}_{D, n}\left(E_{K}\right)}{\bar{y}_{D, x}}
$$

where the choice of reference radiation was made to replicate the use of x-rays by Baiocco et al. [63]. As was alluded to in Chapter 1, extrapolating the results from neutron studies of small animals to humans can be problematic. Indeed, previously published ICRP weighting factors below $1 \mathrm{MeV}$ were considerably larger than current recommendations because earlier small scale studies underestimated the effects of neutron capture gammas relative to protons from (direct) elastic scattering [10]. Thus, it is important to study neutrons on the scale of interest. However, TS codes are time-consuming and confined almost entirely to studies in water. To circumvent these limitations, in this work $\mathrm{CH}$ simulations were performed upstream to both narrow the scope of the TS simulations and to allow for the use of human tissue-equivalent material. From the $\mathrm{CH}$ simulations, the primary spectra (kinetic energies at the point of liberation) of the secondary species and their relative dose contributions were obtained. This then allowed for the spectra method of $\bar{y}_{D}$ calculation (Equation 4.9) to be used for tracks analyzed via the weighted track-sampling algorithm (Section 4.2.2). Qualitative agreement was sought between the predicted RBE results and published radiation 


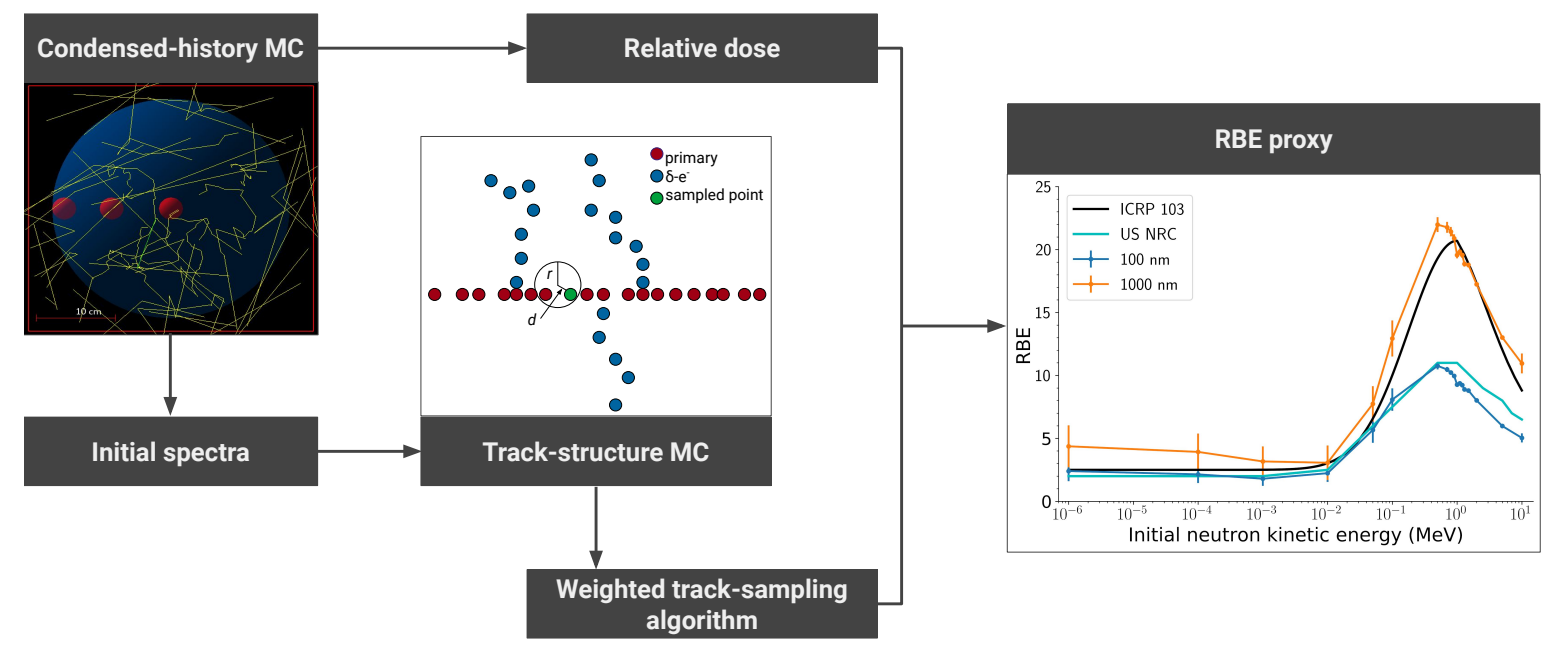

Figure 5.1: A simplified schematic of the neutron microdosimetry simulation pipeline developed in this work. The initial spectra and relative dose contributions of the secondary species produced by mono-energetic neutrons were collected within three scoring volumes centred at different depths within a human tissue-equivalent phantom using CHMC (Geant4). The spectra were then used to determine the initial energy distribution of particle tracks that would be generated using TSMC (Geant4-DNA). Tracks were analysed individually via the weighted track-sampling algorithm and the $\bar{y}_{D}$ value of each relevant spectra was obtained for multiple sampling sphere diameters. Combining the individual $\bar{y}_{D}$ values with their appropriate relative dose contributions yielded the overall neutron $\bar{y}_{D}$ for each volume as a function of energy and sampling sphere diameter. Comparing neutron $\bar{y}_{D}$ to that of a reference radiation yielded a prediction of neutron $\mathrm{RBE}$ in accordance with the theory of dual radiation action.

weighting factors, as this would provide evidence that trends in the underlying data sets could be linked to observed variations in neutron RBE. Thus, conclusions could be drawn on the physics responsible for the energy dependence of neutron RBE. An overview of the workflow is given in Fig. 5.1.

\subsection{Condensed history simulations}

\subsubsection{Geometry}

The phantom geometry was a customized version of the four-component soft tissueequivalent sphere originally recommended by the International Commission on Radiation Units and Measurements (ICRU) for use in radiation safety and dosimetry applications [89]. Intended to approximate a human adult torso, this ICRU-4 sphere is $30 \mathrm{~cm}$ in diameter and comprised of hydrogen, carbon, nitrogen, and oxygen in the proportions detailed in Table 5.1. For these simulations, the version of the ICRU-4 
TABLE 5.1: Elemental composition of the ICRU-4 sphere and the natural abundance of each isotope.

\begin{tabular}{cccc}
\hline Element & $\begin{array}{c}\text { Elemental composition } \\
(\% \text { by mass })\end{array}$ & Isotope & $\begin{array}{c}\text { Natural abundance } \\
(\%)\end{array}$ \\
\hline $\mathrm{H}$ & 10.1 & ${ }^{1} \mathrm{H}$ & 99.98 \\
& & ${ }^{2} \mathrm{H}$ & 0.02 \\
$\mathrm{C}$ & 11.1 & ${ }^{12} \mathrm{C}$ & 98.9 \\
& & ${ }^{13} \mathrm{C}$ & 1.1 \\
$\mathrm{~N}$ & 2.6 & ${ }^{14} \mathrm{~N}$ & 99.6 \\
& & ${ }^{15} \mathrm{~N}$ & 0.4 \\
$\mathrm{O}$ & 76.2 & ${ }^{16} \mathrm{O}$ & 99.76 \\
& & ${ }^{17} \mathrm{O}$ & 0.04 \\
& & ${ }^{18} \mathrm{O}$ & 0.20 \\
\hline
\end{tabular}

material available from the Geant4 Material Database could not be used as it did not incorporate thermal scattering data for neutrons with hydrogen. The material was therefore rebuilt with the appropriate hydrogen data according to the same element weight fractions, density, and other parameters as before. Consistent with the work of Baiocco et al. [63], three spherical scoring volumes of radius $1.5 \mathrm{~cm}$ made of the same modified ICRU-4 material were centred along a single axis at radial distances of $0 \mathrm{~cm}, 7.5 \mathrm{~cm}$, and $13.5 \mathrm{~cm}$ from the phantom's centre. Throughout the rest of this thesis, these volumes will often be referred to as the inner, intermediate, and outer scoring volumes, respectively. The phantom was placed in a $40 \mathrm{~cm}$ diameter sphere of low density material that is used to approximate a vacuum in Geant4. The full geometry is shown in Fig. 5.2.

\subsubsection{Irradiation conditions}

Mono-energetic neutron sources with initial kinetic energies between $1 \mathrm{eV}$ and $10 \mathrm{MeV}$ were distributed uniformly across the surface of the outer vacuum-like sphere and their emissions were directed inwards towards the phantom according to a cosine-law angular distribution. It was empirically determined that $\sim 10^{10}$ neutrons were required to achieve smooth spectra for important secondary particles, i.e. those that contribute more than $\sim 1 \%$ of the relative dose for a given initial neutron energy and scoring volume. To meet this requirement and provide a means of investigating statistical variations between individual runs, six simulations of $2 \times 10^{9}$ neutrons were performed for each chosen neutron energy. This process was repeated for $250 \mathrm{keV}$ x-rays. 


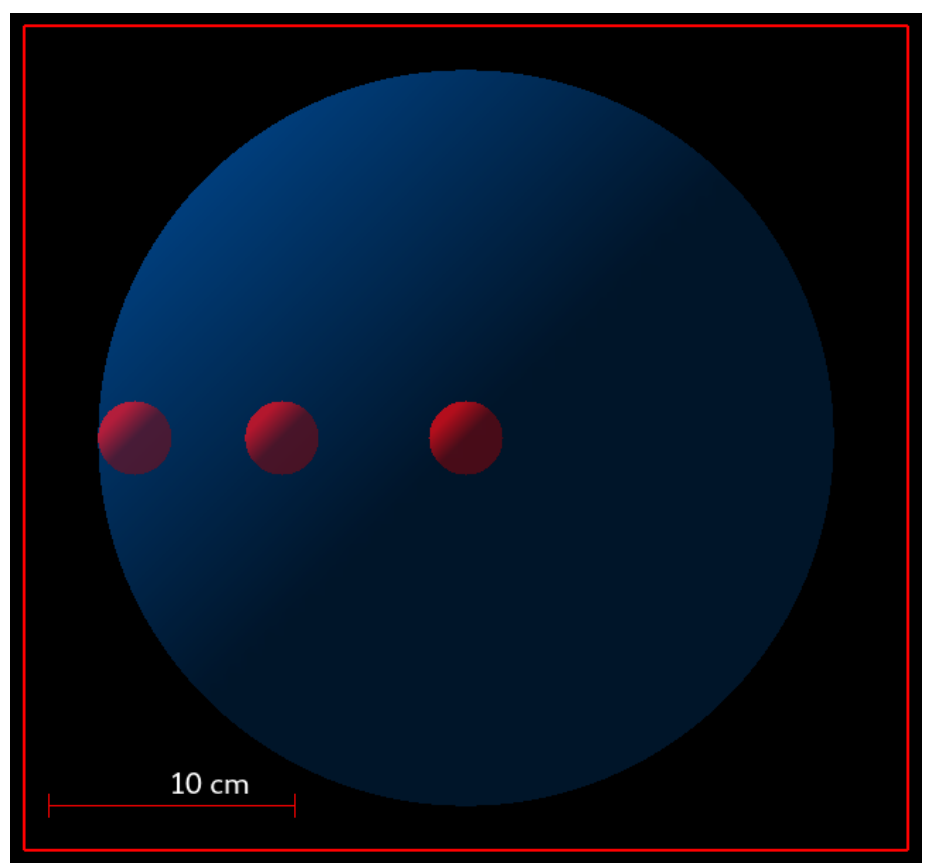

Figure 5.2: Geometry used in the $\mathrm{CH}$ simulations. Three $1.5 \mathrm{~cm}$ radius spherical scoring volumes (shown in red) were placed at various depths in the ICRU-4 sphere (shown in blue). The material composition is given in Table 5.1. Mono-energetic neutron sources were placed externally to the sphere in the surrounding low density gas (shown in black).

\subsubsection{Physics}

The Geant4 ParticleHP models were used to describe hadronic elastic scattering and inelastic processes (scattering, neutron capture, fission). These models rely on tabulated data from the US Evaluated Nuclear Data Files (ENDF/B) for both cross sections and final state determination [34]. For more details on the latest library used in Geant4 10.04 (ENDF/B-VII.1), see Chadwick et al. [90]. Below $4 \mathrm{eV}$, an additional elastic scattering dataset (G4ParticleHPThermalScattering) was incorporated to improve the transport of thermal neutrons.

The Lawrence Livermore evaluated data libraries for photons [91], electrons [92], and atomic relaxation [93] (G4EmLivermorePhysics) were used to handle electromagnetic processes such as Compton scattering, bremsstrahlung, and Auger cascades. Default classes were included to handle less significant reactions such as free neutron decay (G4DecayPhysics) and the decay of radioactive isotopes (G4RadioactiveDecay Physics). Heavy ion transport models were unnecessary for reasons that will be discussed below. 


\subsubsection{Scoring}

\section{Spectra and relative dose}

The secondary particles generated by neutrons in the energy range studied are gammas, protons, deuterons, tritons, alphas and beryllium, boron, carbon, nitrogen, and oxygen ions. The secondary gammas produce electrons and positrons. Due to the short range of the secondary protons and heavy charged particles and their low radiative yield (the fraction of kinetic energy lost to bremsstrahlung and characteristic x-ray production), neutron dose is approximately equivalent to electronic kerma [21]. This allows for the use of the local approximation (Section 3.3.2), which is believed to hold for neutron fields in human tissue up to about $20 \mathrm{MeV}$ [94]. In MC, the local approximation is accomplished by killing particles at their point of generation and considering their energies to be deposited on the spot.

Particle spectra were stored in histograms with 500 logarithmically spaced bins between $1 \mathrm{eV}$ and $10 \mathrm{MeV}$ and their dose contributions were recorded when particles were generated within the scoring volumes. However, special consideration was given to high energy ( $\gtrsim 1 \mathrm{MeV}$ ) electrons because Geant4-DNA does not allow for the transport of electrons with kinetic energies greater than $1 \mathrm{MeV}$. Thus, these electrons could not be killed immediately after generation and were instead tracked down to $1 \mathrm{MeV}$, at which point they could be considered to deposit their energies locally. Simulation results were output to .csv files and plotted via external Python3 scripts.

\section{Explicit dose calculation}

To investigate the validity of the local approximation for the $\mathrm{CH}$ simulations, the dose deposited by secondary protons and electrons was explicitly calculated for three initial neutron energies: $1 \mathrm{eV}, 1 \mathrm{MeV}$, and $10 \mathrm{MeV}$. The simulation parameters were identical to those discussed in this section, except that the generated electrons and protons were instead simulated down to $0 \mathrm{eV}$. Care was taken to ensure that the dose deposited by proton-generated electrons was distinguished from the dose deposited by the gamma-generated electrons.

\subsection{Track-structure simulations}

\subsubsection{Geometry and irradiation conditions}

Source particles for the TS simulations were obtained by sampling the secondary particle distributions obtained during the $\mathrm{CH}$ phase. This was accomplished by using the histogram-processing functionality of the G4GeneralParticleSource (GPS) class. 
For every (i) particle type, (ii) scoring volume, and (iii) initial neutron energy, the corresponding distribution was sampled 10,000 times to generate a set of source particles for a simulation of 10,000 independent tracks. Within each simulation, the sampled particles were emitted isotropically from the centre of a $40 \mathrm{~km}$ radius (i.e. semi-infinite) sphere made up of liquid water (G4_Water from the National Institute of Standards and Technology; NIST database).

\subsubsection{Physics}

A custom physics constructor was written for the handling of charged particle interactions in the TS code. Between $10 \mathrm{keV}$ and $1 \mathrm{MeV}$, an implementation of the Emfietzoglou model of the dielectric function of liquid water [95] (based on the first Born approximation) with semi-empirical low-energy corrections [96] was employed for excitation and ionization events. At lower energies, a more detailed set of low-energy corrections to the Emfietzoglou model [97] was used. Elastic scattering interactions were handled below $10 \mathrm{keV}$ by a screened Rutherford analytical model supplemented by an experimentally-derived screening parameter from Uehara et al. [98] for improved low-energy transport [16]. A partial wave interpolated model [99] was used between $10 \mathrm{keV}$ and $1 \mathrm{MeV}$. A vibrational excitation model based on data from Michaud et al. [100] was used up to $100 \mathrm{eV}$, while an electron attachment model based on data from Melton [101] was used up to $13 \mathrm{eV}$ [36]. Atomic relaxation was handled by the same Livermore models as described in Section 5.2.3. All fluorescent gammas were killed because it was assumed that their energies were radiated far enough away that their energy depositions were no longer correlated with their particle tracks. Electrons were tracked down to $10 \mathrm{eV}$ at which point they were killed and their energies deposited locally. The relevant Geant4-DNA model classes are summarized in Table 5.2.

The elastic nuclear scattering of charged particles heavier than electrons was modelled using a non-relativistic classical approach [102]. These theories are valid down to $100 \mathrm{eV}$ for all particles and extend up to $1 \mathrm{MeV}$ for protons and hydrogen atoms and up to $10 \mathrm{MeV}$ for helium atoms of all charge states. Proton and hydrogen ionization models developed using Born and Bethe theories along with the dielectric formalism of liquid water [103] were employed. Between $500 \mathrm{keV}$ and $10 \mathrm{MeV}$, this treatment is sufficient; however, a semi-empirical approach based on work by Rudd et al. [104] was necessary to extend the validity down to $100 \mathrm{eV}$ [35]. The Born and Bethe-based theoretical approach was also sufficient for the description of excitation interactions between $500 \mathrm{keV}$ and $100 \mathrm{MeV}$. A method by Miller and Green [105] involving a speed-scaling of electron excitation cross sections was used to extend the excitation description down to $10 \mathrm{eV}$ [103]. The analytical Dingfelder model [103] of charge exchange processes governed electron capture by protons and electron loss by 
TABLE 5.2: Physics models used in the TS simulations for electron transport.

\begin{tabular}{lll}
\hline Process & Model class & Energy range \\
\hline Elastic scattering & G4DNAUeharaScreenedRutherfordElasticModel & $10 \mathrm{eV}-10 \mathrm{keV}$ \\
& G4DNAChampionElasticModel & $10 \mathrm{keV}-1 \mathrm{MeV}$ \\
Electronic excitation & G4DNAEmfietzoglouExcitationModel & $10 \mathrm{eV}-10 \mathrm{keV}$ \\
& G4DNABornExcitationModel & $10 \mathrm{keV}-1 \mathrm{MeV}$ \\
Ionization & G4DNAEmfietzoglouIonisationModel & $10 \mathrm{eV}-10 \mathrm{keV}$ \\
& G4DNABornIonisationModel & $10 \mathrm{keV}-1 \mathrm{MeV}$ \\
Vibrational excitation & G4DNASancheExcitationModel & $10 \mathrm{eV}-100 \mathrm{eV}$ \\
Attachment & G4DNAMeltonAttachmentModel & $10 \mathrm{eV}-13 \mathrm{eV}$ \\
\hline
\end{tabular}

neutral hydrogen between $100 \mathrm{eV}$ and $100 \mathrm{MeV}$ [35]. The excitation, ionization, and charge exchange processes of helium atoms were described via the same theoretical bases as the protons and hydrogen atoms but with a few modifications such as effective charge and speed scaling [35]. The range of validity for non-elastic helium atom interactions was from $1 \mathrm{keV}$ to $400 \mathrm{MeV}$. The relevant Geant4-DNA model classes are summarized in Tables 5.3 and 5.4 for hydrogen and helium, respectively.

\subsubsection{Scoring}

Every interaction that involved a non-zero local energy deposit (i.e. every transfer point) was identified during the generation of each primary track. At each transfer point, the incident particle's kinetic energy, initial position, and final position were recorded, along with the total energy deposited. The correlated secondary tracks were scored in the same manner and thus the full track structure associated with each primary track was obtained.

The weighted track sampling algorithm (described in Section 4.2.2) was implemented using sampling spheres with diameters of $2 \mathrm{~nm}, 10 \mathrm{~nm}, 30 \mathrm{~nm}, 100 \mathrm{~nm}$, and $1000 \mathrm{~nm}$. All tracks were sampled $M=1000$ times for each sampling radius. The transfer point density weight $w_{\mathrm{tp}, i j}$ of each sampled transfer point $i$ of track $j$ was equal to the reciprocal of the number of transfer points in its corresponding sampling sphere. After the track had been sampled $M$ times, the weights were applied to the corresponding imparted energies and squared imparted energies to calculate the weighted lineal energy and weighted square lineal energy of the track: 
TABLE 5.3: Physics models used in the TS simulations for proton and neutral hydrogen transport. ${ }^{*}$ valid for neutral hydrogen.

\begin{tabular}{lll}
\hline Process & Model class & Energy range \\
\hline Nuclear scattering & G4DNAIonElasticModel & $100 \mathrm{eV}-1 \mathrm{MeV}$ \\
Electronic excitation & G4DNAMillerGreenExcitationModel & $10 \mathrm{eV}-500 \mathrm{keV}$ \\
& G4DNABornExcitationModel & $500 \mathrm{keV}-100 \mathrm{MeV}$ \\
Ionization & G4DNARuddIonisationModel & $10 \mathrm{eV}-500 \mathrm{keV}$ \\
& G4DNABornIonisationModel & $500 \mathrm{keV}-100 \mathrm{MeV}$ \\
Electron capture & G4DNADingfelderChargeDecreaseModel & $100 \mathrm{eV}-10 \mathrm{MeV}$ \\
Charge increase* & G4DNADingfelderChargeIncreaseModel & $100 \mathrm{eV}-10 \mathrm{MeV}$ \\
\hline
\end{tabular}

TABLE 5.4: Physics models used in the TS simulations for helium atom transport. ${ }^{*}$ valid for neutral and singly-ionized helium. $\dagger$ valid for singly-ionized and doubly-ionized helium.

\begin{tabular}{lll}
\hline Process & Model class & Energy range \\
\hline Nuclear scattering & G4DNAIonElasticModel & $100 \mathrm{eV}-1 \mathrm{MeV}$ \\
Electronic excitation & G4DNAMillerGreenExcitationModel & $1 \mathrm{keV}-10 \mathrm{MeV}$ \\
Ionization & G4DNARuddIonisationModel & $10 \mathrm{eV}-10 \mathrm{MeV}$ \\
Charge increase* $^{*}$ & G4DNADingfelderChargeIncreaseModel & $1 \mathrm{keV}-10 \mathrm{MeV}$ \\
Charge decrease $^{\dagger}$ & G4DNADingfelderChargeDecreaseModel & $1 \mathrm{keV}-10 \mathrm{MeV}$ \\
\hline
\end{tabular}

$$
y_{j}=\frac{\sum_{i=1}^{M} \epsilon_{i j} w_{\mathrm{tp}, i j}}{\bar{\ell}_{j} \sum_{i=1}^{M} w_{\mathrm{tp}, i j}} ; \quad y_{j}^{2}=\frac{\sum_{i=1}^{M} \epsilon_{i j}^{2} w_{\mathrm{tp}, i j}}{\bar{\ell}_{j}^{2} \sum_{i=1}^{M} w_{\mathrm{tp}, i j}}
$$

where $\bar{\ell}=4 r / 3$ was the mean chord length of the sampling sphere that was used.

In order to calculate the $\bar{y}_{D}$ of the particle species, an associated volume weight $\omega_{a v, j}$ was calculated for each track $j$. This calculation was done by first creating a vector that contained the final positions of all interactions in a given track. These positions were then translated into coordinates on a grid composed of voxels with side length $2 r / 3$. The list of voxel coordinates was then filtered to eliminate redundant entries. Each of the remaining voxels was set as the centre of a cube with 27 subdivisions by padding along $x, y$, and $z$ (creating a mesh). Once all cubes had been formed, repeated coordinates were filtered out and the volume of the remaining subdivisions was calculated. In this way, a cube with side length $2 r$ (i.e. the cube circumscribed by the 
sampling sphere) was centred on each position and the union of the set was calculated and set as the associated volume weight. $\bar{y}_{D}$ was then calculated by multiplying the associated volume $\omega_{a v, j}$ of each track with its weighted lineal energy $y_{j}$ and weighted square lineal energy $y_{j}^{2}$, and summing over all $N$ tracks:

$$
\bar{y}_{D}=\frac{\sum_{j=1}^{N} y_{j}^{2} \frac{\omega_{a v, j}}{\sum_{j=1}^{N} \omega_{a v, j}}}{\sum_{j=1}^{N} y_{j} \frac{\omega_{a v, j}}{\sum_{j=1}^{N} \omega_{a v, j}}}
$$

Finally, for each sampling sphere radius, the overall neutron $\bar{y}_{D}$ was determined by combining the species-specific results in a weighted sum wherein each secondary contribution was multiplied by the fraction of the overall dose it deposited (Equation 4.9).

\subsection{Final results}

The energy-dependence of microdosimetrically-predicted neutron RBE was determined for each scoring volume depth as a function of sampling diameter by dividing the $\bar{y}_{D}$ values for each neutron energy by the corresponding values determined for $250 \mathrm{keV}$ xrays (Equation 5.1). Results were compared with published experimental and numerical values. Special consideration was given to the radiation weighting factors published by the ICRP and the US NRC. 



\section{Chapter 6}

\section{Results}

\subsection{Charged particle spectra}

\subsubsection{Neutrons}

The spectra of secondary gammas, protons, deuterons, tritons, alphas and beryllium, boron, carbon, nitrogen, and oxygen ions produced by mono-energetic neutron sources with initial kinetic energies of $1 \times 10^{-6}, 1 \times 10^{-4}, 1 \times 10^{-3}, 1 \times 10^{-2}, 5 \times 10^{-2}, 0.1,0.5,0.7$, $0.8,0.9,1,1.1,1.2,1.3,1.5,2,5$, and $10 \mathrm{MeV}$ were collected in all three scoring volumes. The spectra of the tertiary and higher order electrons and positrons stemming from the secondary gammas were also obtained. Fig. 6.1 shows the spectra for the $1 \mathrm{keV}$, $1 \mathrm{MeV}$, and $10 \mathrm{MeV}$ neutron sources in all three of the scoring volumes to illustrate trends in the data. Tritons, beryllium ions, and boron ions were excluded from the graphs because their numbers were negligible.

Generally, with increasing depth (Fig. 6.1 left to right), a lower proportion of protons and heavy ions were liberated relative to gammas. Furthermore, the spectra of these protons and ions was skewed towards lower energies for deeper scoring volumes. A minimal depth dependence across all energies was observed in the shape of the gamma spectra.

Increasing the incident neutron energy from $1 \mathrm{eV}$ to $2 \mathrm{MeV}$ resulted in higher proportions of protons and heavy ions (Fig. 6.1 top row to middle row). At higher energies, the proportion of gammas and some heavy ions increased while the proton proportion decreased with increasing energy (Fig. 6.1 bottom row).

The spectra of electrons liberated by secondary gammas during irradiation by $1 \mathrm{keV}$, $1 \mathrm{MeV}$, and $10 \mathrm{MeV}$ neutrons in each of the scoring volumes are shown in Fig. 6.2. Clear peaks occurred at energies of $1 \mathrm{keV}$ and $1 \mathrm{MeV}$. Neither neutron energy nor phantom depth had much of an effect on the electron spectrum for neutron energies below a few $\mathrm{MeV}$ (Fig. 6.2 top row to middle row). However, as the neutron energy was increased to $10 \mathrm{MeV}$, the relative height of the $1 \mathrm{MeV}$ peak was reduced (Fig. 6.2 bottom row). The positron spectra are not shown as their numbers were negligible. 


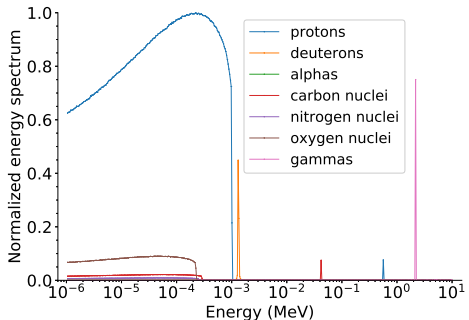

(a)

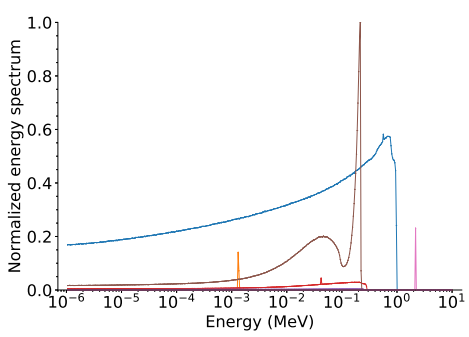

(d)

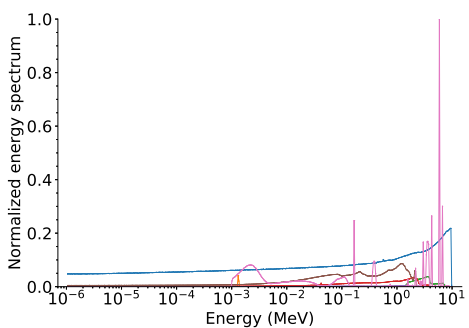

(g)

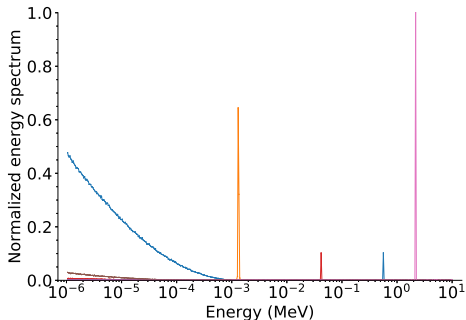

(b)

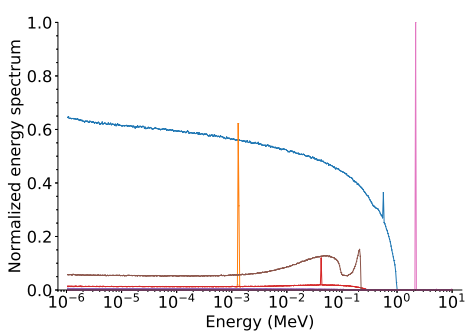

(e)

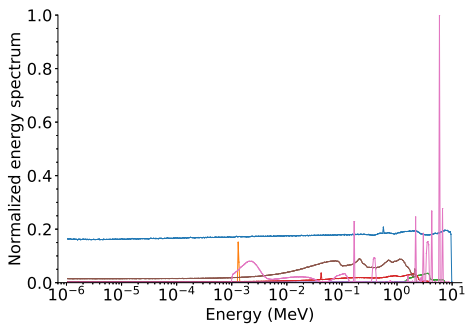

(h)

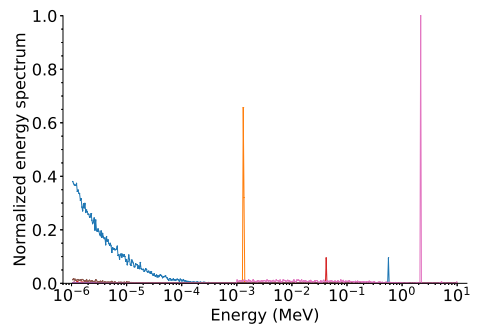

(c)

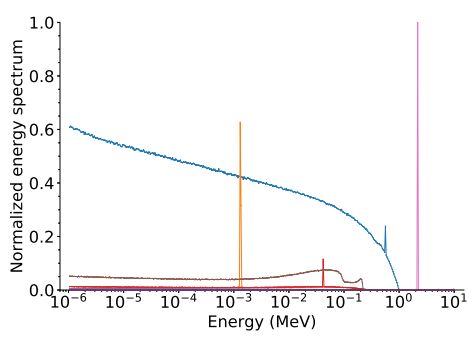

(f)

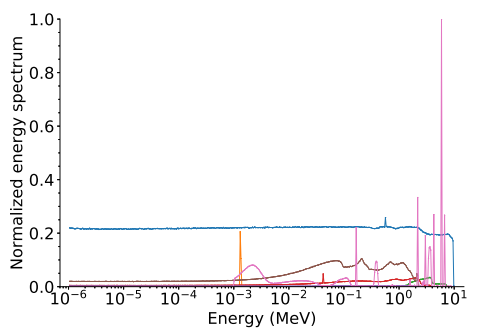

(i)

Figure 6.1: Secondary charged particle spectra produced in each of the three scoring volumes by three mono-energetic neutron sources. Scoring volumes are shown in increasing depth from left to right; (left) outer, (middle) intermediate, (right) inner. Neutron energies are shown in increasing energy from top to bottom; (top) $1 \mathrm{keV}$, (middle) $1 \mathrm{MeV}$, (bottom) $10 \mathrm{MeV}$. Spectra are normalised to the most probable particle type and energy. 


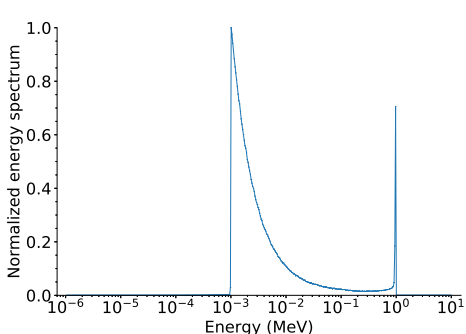

(a)

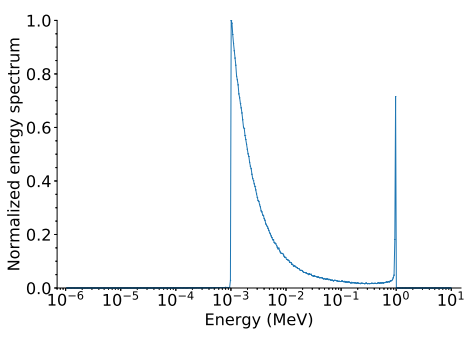

(d)

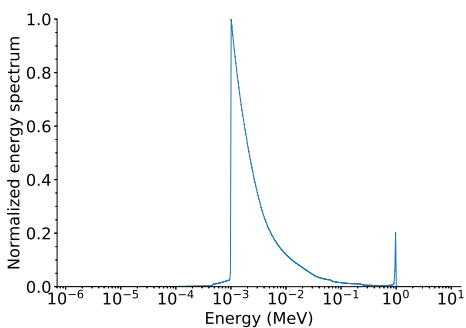

(g)

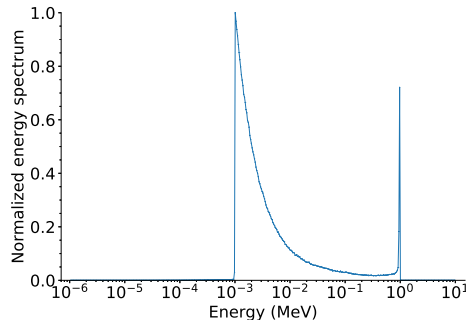

(b)

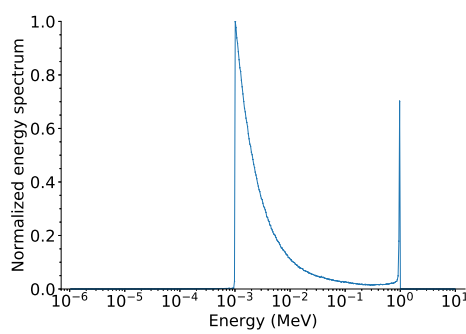

(e)

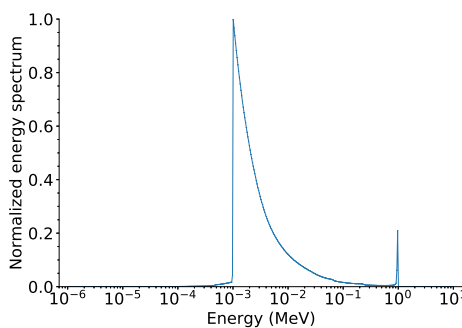

(h)

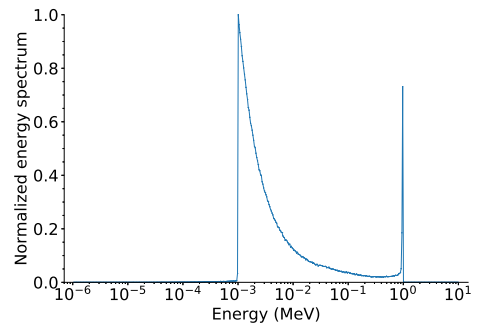

(c)

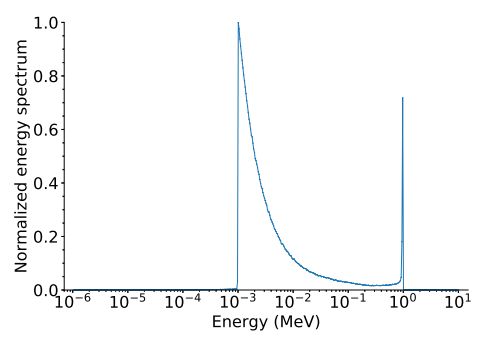

(f)

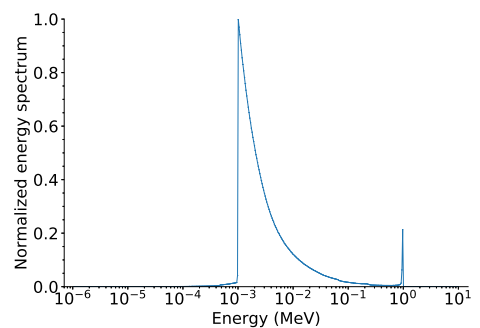

(i)

Figure 6.2: Spectra of electrons produced by secondary gammas in each of the three scoring volumes after irradiation by mono-energetic neutron sources. Scoring volumes are shown in increasing depth from left to right; (left) outer, (middle) intermediate, (right) inner. Neutron energies are shown in increasing energy from top to bottom; (top) $1 \mathrm{keV}$, (middle) $1 \mathrm{MeV}$, (bottom) $10 \mathrm{MeV}$. Spectra are normalised to the most probable electron energy. 


\subsection{2 $250 \mathrm{keV}$ x-rays}

At $250 \mathrm{keV}$, the only charged particles of interest are electrons produced by the primary photons or by fluorescence photons. The normalized spectra of these electrons were gathered in each of the three scoring volumes and are plotted in Fig. 6.3. Although a small number of electrons were produced near $250 \mathrm{keV}$, nearly all were liberated with energies under about $120 \mathrm{keV}$. The peak near $120 \mathrm{keV}$ was most pronounced in the outer scoring volume. Towards the centre of the phantom, the peak was diminished as more low energy electrons were generated across a relatively broader, flatter spectrum.

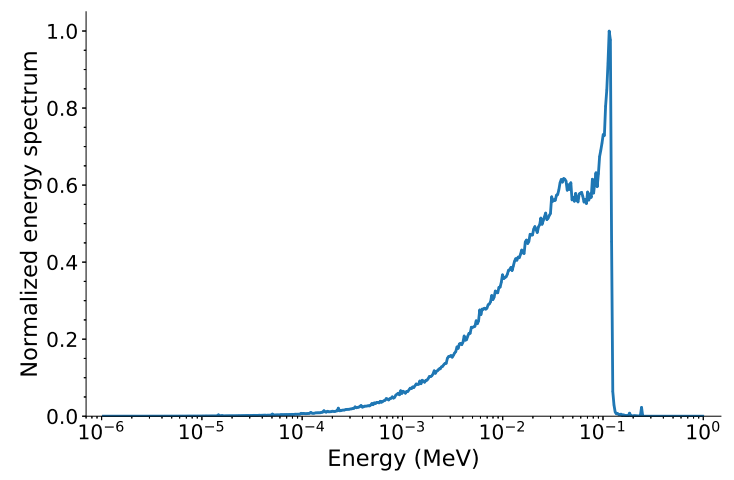

(a)

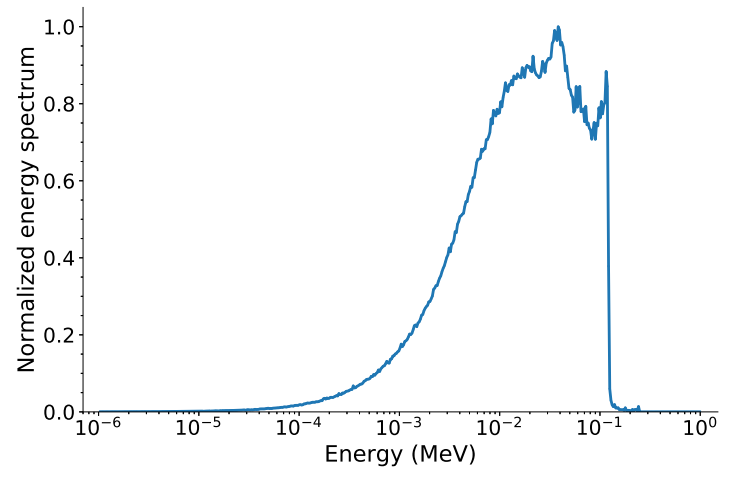

(b)

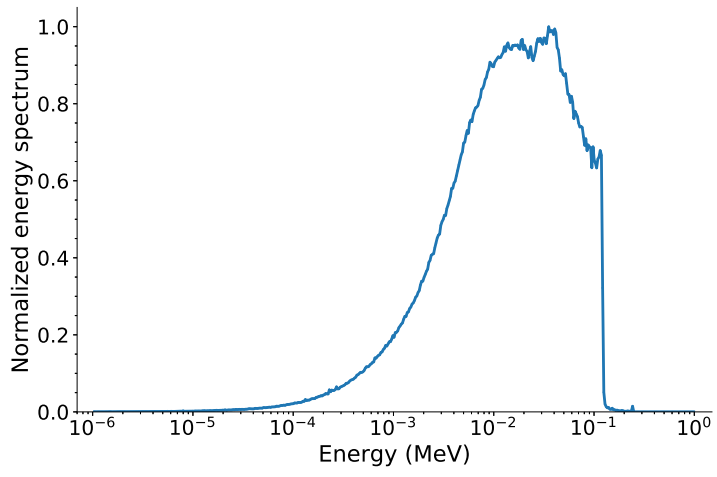

(c)

FIGURE 6.3: The secondary electron spectra produced by $250 \mathrm{keV}$ $\mathrm{x}$-rays in each of the scoring volumes. (a) Outer scoring volume, (b) intermediate scoring volume, and (c) inner scoring volume.

\subsection{Relative dose contributions}

The relative dose contributions ( $d_{i}$ from Equation 4.9) of the secondary species were determined for the neutron energies listed above in each of the three scoring volumes. As can be seen in Fig. 6.4, most of the dose was deposited by electrons at low energies. The dominance was especially pronounced in the innermost scoring volume, where the 
electrons account for approximately all of the dose until about $100 \mathrm{keV}$ (Fig. 6.4(c)). For all volumes, the proton contribution eventually overtook the electron contribution at high neutron energies, and together the two particles were responsible for a large majority of the dose across the entire range of neutron energies. The deeper the scoring volume, the higher the energy of the proton-electron cross-over point and the larger the predominance of electrons below it. At neutron energies of a few hundred $\mathrm{keV}$ and above, the oxygen contribution also became relevant, peaking at about $18 \%$ of the total dose in the outer scoring volume for $1 \mathrm{MeV}$ neutrons (Fig. 6.4(a)). Alphas were only relevant at 5 and $10 \mathrm{MeV}$, making up between $1 \%$ and $6 \%$ of the dose in this energy range depending on the scoring volume (Section 7.2). Like the rest of the heavy ions, carbons were more prevalent at all energies for scoring volumes closer to the surface. In the outer volume, carbons are responsible for about $1-2 \%$ of the dose across the whole energy range (Fig. 6.4(a)), while they contribute far less below $1 \mathrm{MeV}$ for the other two volumes (Figs. 6.4(b) and 6.4(c)).

The results of the explicit dose calculation are also shown in Fig. 6.4, denoted by $\mathrm{X}$ symbols of the corresponding colour for each of the particles. For all proton and heavy ion data points, the local approximation results were in strong agreement with the explicit calculations. However, the local approximation underestimated the electron contribution for $1 \mathrm{MeV}$ and $10 \mathrm{MeV}$ neutrons, with the effect more pronounced at $10 \mathrm{MeV}$. Excepting the overestimation of the $1 \mathrm{eV}$ proton contribution in the intermediate scoring volume due to its negligible value, the largest relative change was a $38 \%$ underestimation of the electron contribution in the outer scoring volume for $10 \mathrm{MeV}$ neutrons.

Relative dose contributions are not shown for the $250 \mathrm{keV}$ x-rays as virtually all of the energy was deposited by electrons in each of the volumes.

\subsection{Dose-mean lineal energies}

\subsubsection{Neutron secondaries}

The $\bar{y}_{D}$ of the secondary proton, electron, and alpha particle spectra were calculated via the weighted track-sampling algorithm using spherical sampling volumes with diameters of $2,10,30,100$, and $1000 \mathrm{~nm}$. Tracks were simulated for all proton and electron spectra. Alpha particle tracks were only simulated for the spectra generated by 5 and $10 \mathrm{MeV}$ neutron sources because their contributions were considered negligible at lower neutron energies and thus the increase in computation time was unjustified. As a representative data set, species-specific $\bar{y}_{D}$ values obtained using the $1000 \mathrm{~nm}$ diameter sampling spheres are shown in Fig. 6.5 for all three scoring volumes. Note that Baiocco 


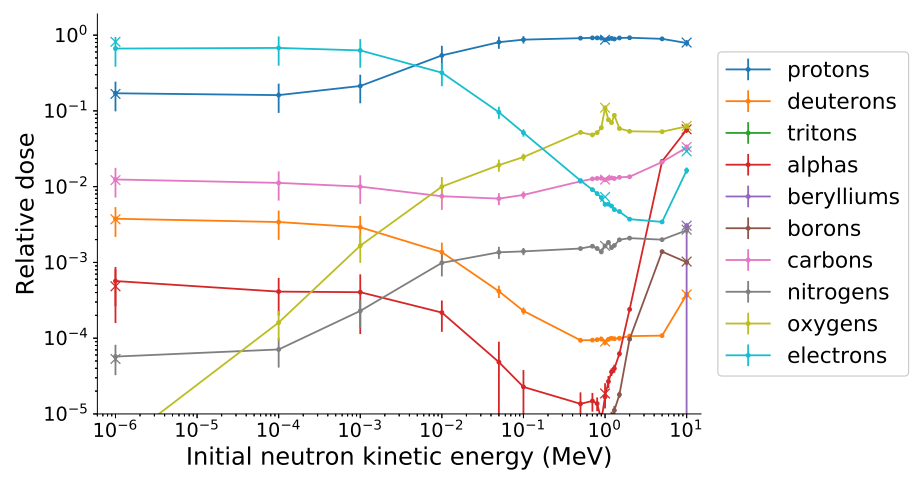

(a)

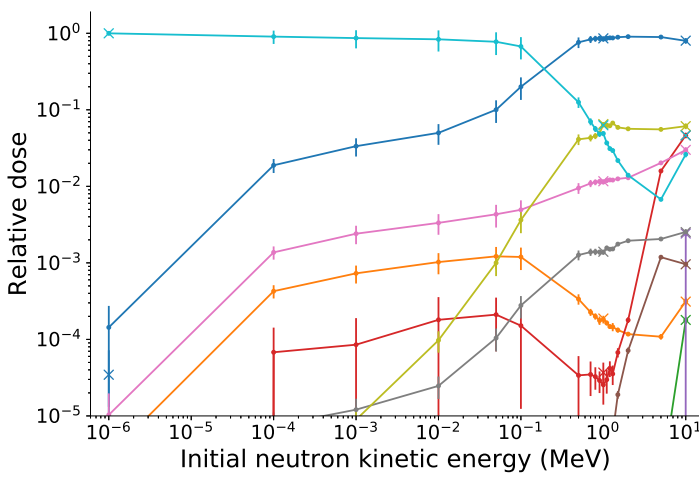

(b)

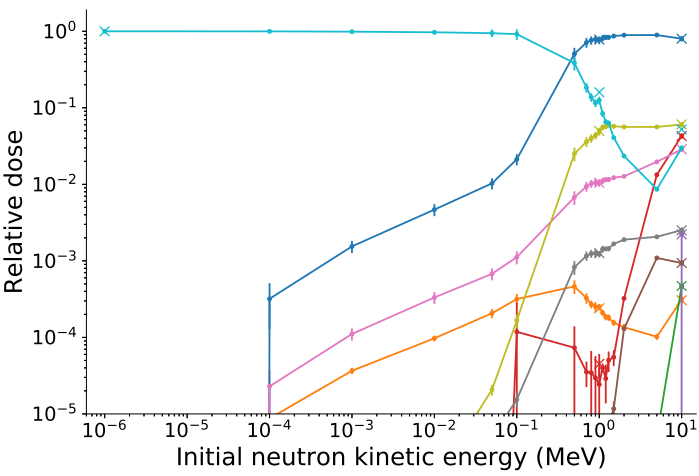

(c)

FiguRE 6.4: Relative dose contributions as calculated by the local approximation for each secondary species produced in all three scoring volumes for a range of mono-energetic neutron sources. Error bars are the standard deviation about the mean of 6 runs. The results of the explicit dose calculation are shown as $\mathrm{X}$ symbols. Lines are drawn to guide the eye. (a) Outer scoring volume, (b) intermediate scoring volume,

(c) inner scoring volume. 
et al. [63] used $1000 \mathrm{~nm}$ diameter spheres in their study. In all scoring volumes, the proton $\bar{y}_{D}$ was larger than the electron $\bar{y}_{D}$ at all neutron energies tested, while the alpha $\bar{y}_{D}$ was far greater still. The electron $\bar{y}_{D}$ was approximately constant at all depths from $1 \mathrm{eV}$ to $2 \mathrm{MeV}$ but rose slightly as the neutron energy increased towards $10 \mathrm{MeV}$. Proton $\bar{y}_{D}$ was approximately constant in the deeper scoring volumes (Figs. 6.5(b) and $6.5(\mathrm{c})$ ), however, there are a few notable features. As neutron energy increased, the $\bar{y}_{D}$ of the proton spectra displayed a minimum. With increasing depth, this minimum occurred at higher energies and was less pronounced. Above the minimum energy the proton $\bar{y}_{D}$ rise towards a local maximum and a subsequent fall-off. The $\bar{y}_{D}$ peak, occurring between 0.5 and $0.8 \mathrm{MeV}$ for all volumes, did not extend much higher than the values at low neutron energies (if at all) but was accentuated by a sharp decrease in $\bar{y}_{D}$ to either side. For the alphas, the $\bar{y}_{D}$ arising from $5 \mathrm{MeV}$ neutrons was greater than that arising from the $10 \mathrm{MeV}$ neutron-generated spectrum in all volumes.

The variation of proton $\bar{y}_{D}$ with sampling sphere diameter is shown in Fig. 6.6. In general, decreasing the sampling diameter resulted in the minimum and peak being less pronounced and occurring at lower energies. For the inner volume, $\bar{y}_{D}$ increased with increasing sampling diameter for all neutron energies, up to $100 \mathrm{~nm}$; although the $30 \mathrm{~nm}$ and $100 \mathrm{~nm}$ results were nearly identical (Fig. 6.6(c)). A further increase to $1000 \mathrm{~nm}$ resulted in a decrease in $\bar{y}_{D}$. Similar trends were seen in the shallower volumes; however, there was some cross-over due to the sharp reduction in the depth of the minimum and slope of the post-peak fall-off with decreasing sampling diameter.

Fig. 6.7 shows the variation of $\bar{y}_{D}$ with sampling diameter for the electron spectra in each of the scoring volumes. Decreasing the sampling volume increased the magnitude without altering its shape in any significant way. The low energy constancy and slight increase at high energies were present in all data sets, although the rise is much less pronounced for the $2 \mathrm{~nm}$ sampling volume. Changes in scoring volume depth had no effect for any of the sampling diameters.

\subsection{2 $250 \mathrm{keV}$ x-rays}

The $\bar{y}_{D}$ values for the secondary electron spectra produced by the $250 \mathrm{keV}$ x-rays are shown in Fig. 6.8 as a function of sampling sphere diameter. As electrons were the only particles produced by the x-rays, the $\bar{y}_{D}$ of their spectra is equivalent to the $\bar{y}_{D}$ of the x-rays. Increasing the sampling diameter caused a significant reduction in the calculated $\bar{y}_{D}$. There was a slight depth dependence, with more interior volumes experiencing marginally higher $\bar{y}_{D}$ values. 


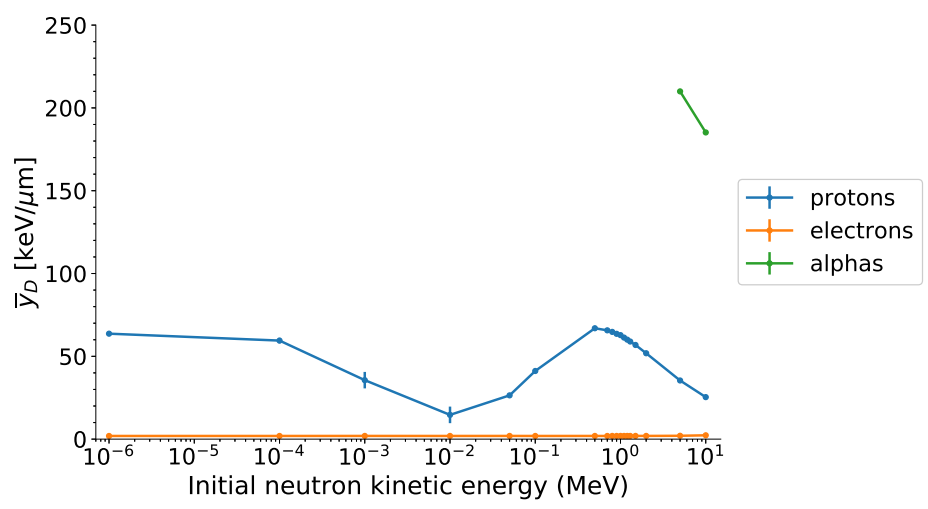

(a)

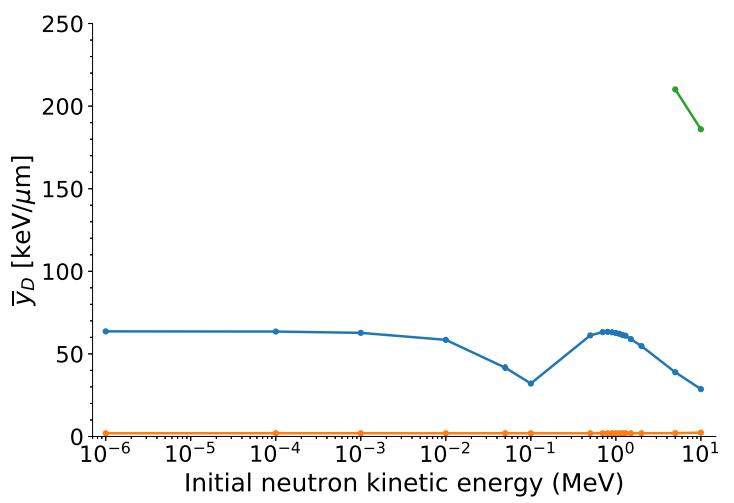

(b)

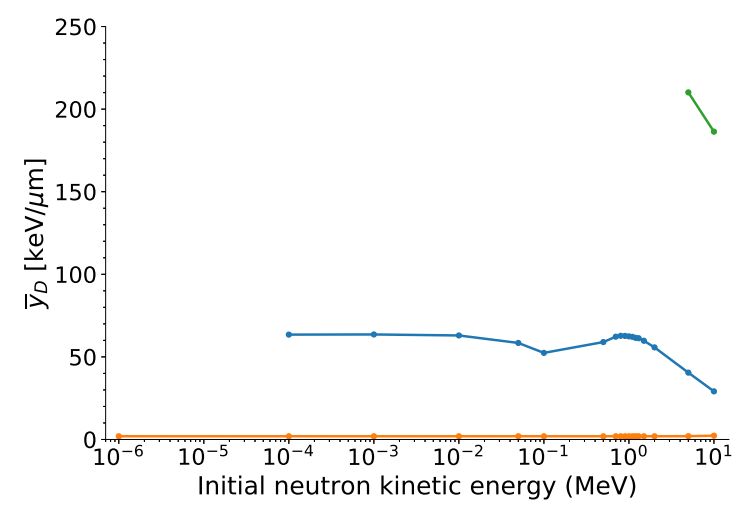

(c)

FiguRE 6.5: Calculated $\bar{y}_{D}$ for the secondary proton, electron, and alpha spectra produced by a range of mono-energetic neutron sources within each scoring volume. Values are given for a spherical sampling diameter of $1000 \mathrm{~nm}$, as an example. Error bars are inter-run standard deviations about the mean of 3 runs. Lines are drawn to guide the eye. (a) Outer scoring volume, (b) intermediate scoring volume, (c) inner scoring volume. 


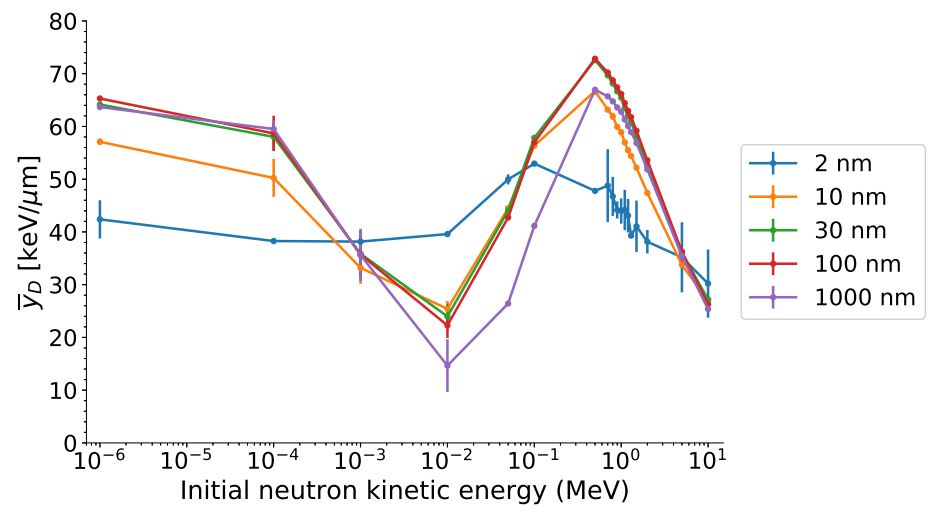

(a)

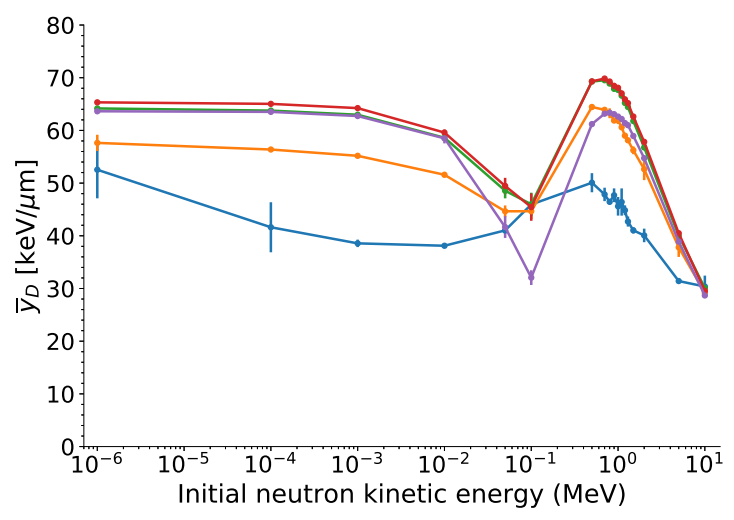

(b)

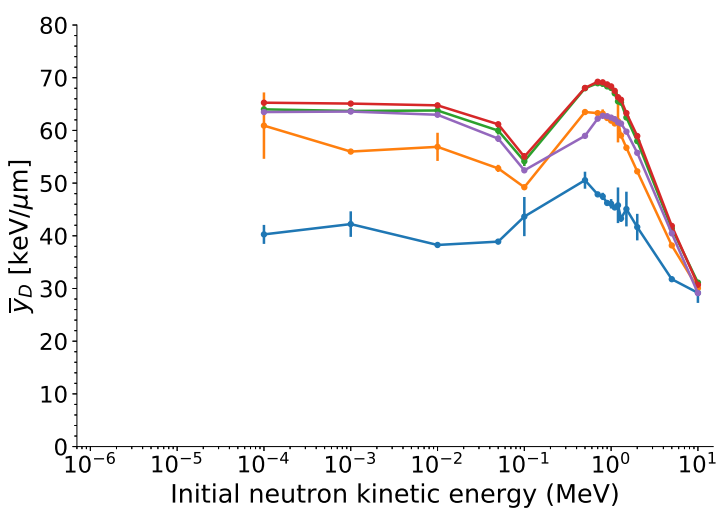

(c)

Figure 6.6: Calculated $\bar{y}_{D}$ values of the proton spectra generated in each scoring volume by a range of mono-energetic neutron sources for several spherical sampling volume diameters. Error bars are inter-run standard deviations about the mean of 3 runs. Lines are drawn to guide the eye. (a) Outer scoring volume, (b) intermediate scoring volume, (c) inner scoring volume. 


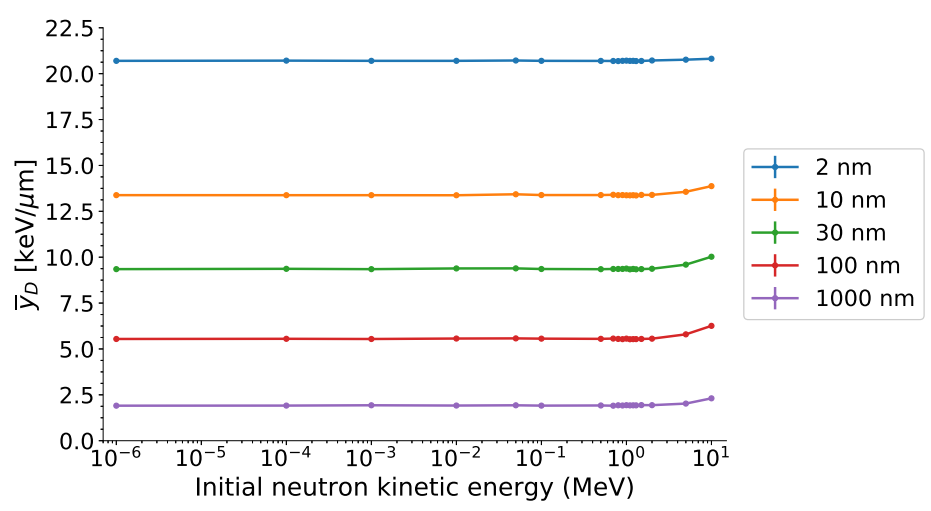

(a)

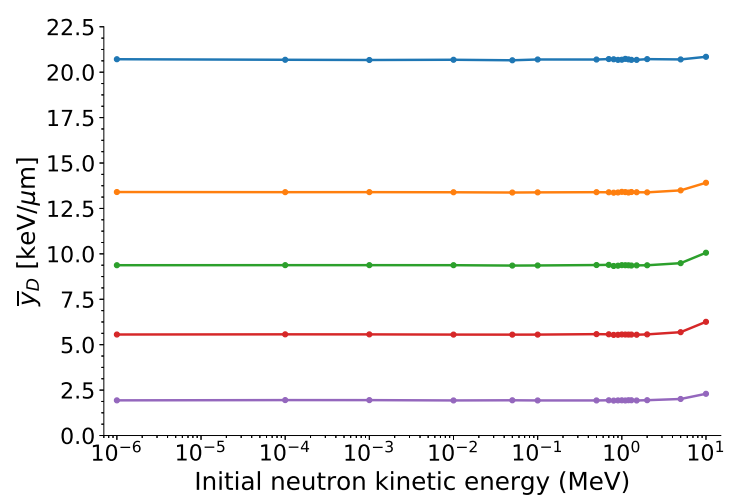

(b)

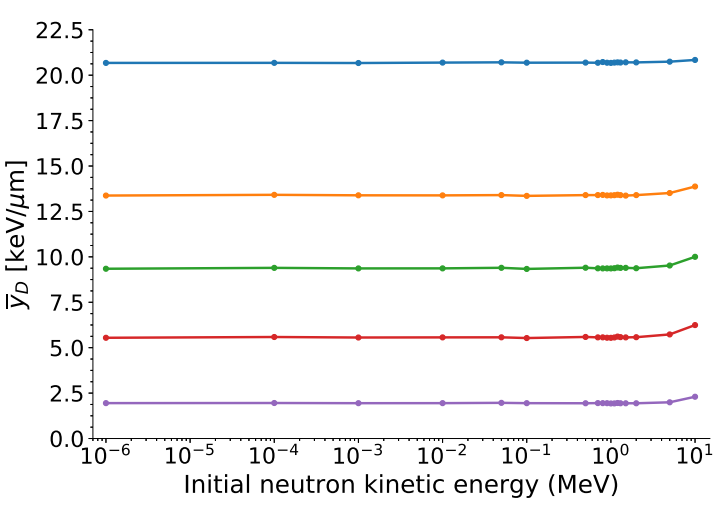

(c)

FiguRE 6.7: Calculated $\bar{y}_{D}$ values of the electron spectra generated in each scoring volume by a range of mono-energetic neutron sources for several spherical sampling volume diameters. Error bars are inter-run standard deviations about the mean of 3 runs. Lines are drawn to guide the eye. (a) Outer scoring volume, (b) intermediate scoring volume, (c) inner scoring volume. 


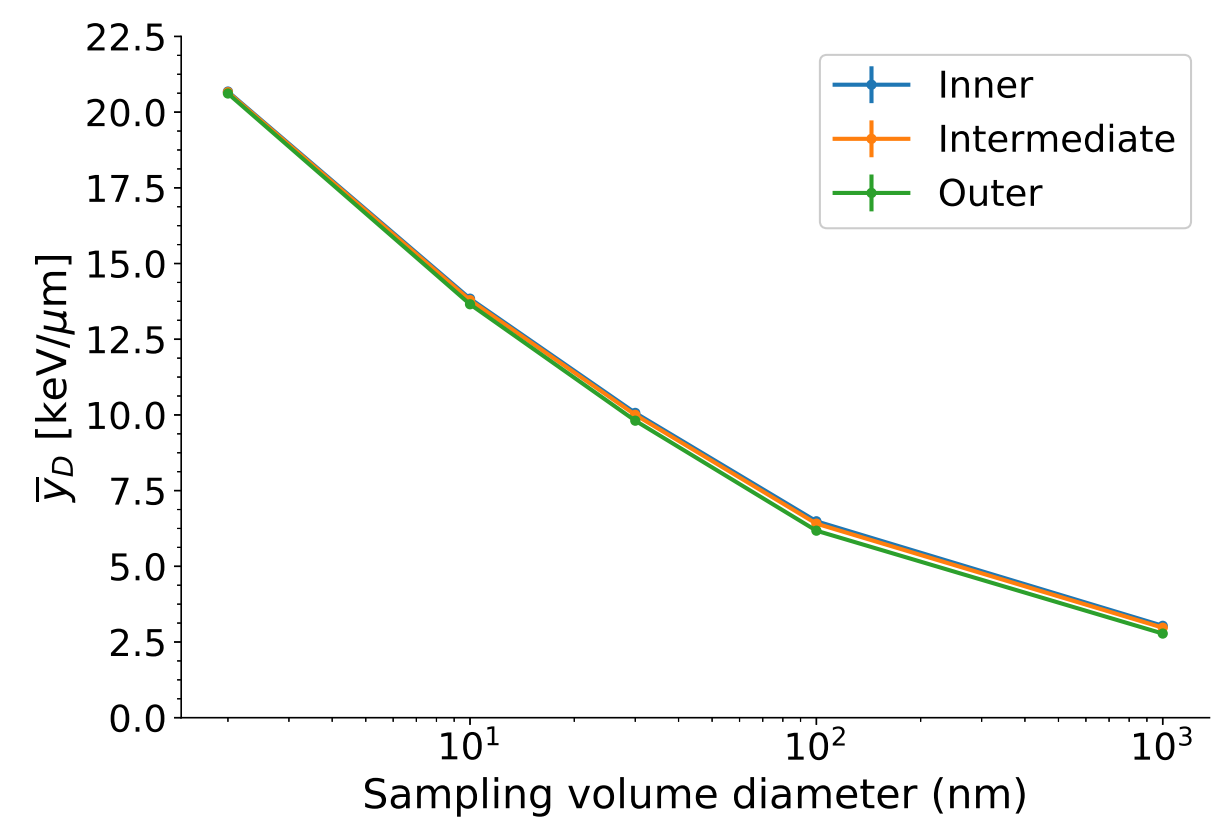

FIgURE 6.8: Calculated $\bar{y}_{D}$ values as a function of spherical sampling volume diameter for the electron spectra produced by $250 \mathrm{keV}$ x-rays (and thus of the x-rays themselves) in each of the scoring volumes. Error bars are inter-run standard deviations about the mean of 3 runs and are contained within the data points. Lines are drawn to guide the eye.

\subsection{Neutron RBE}

Following Equation 4.9, the neutron $\bar{y}_{D}$ values for each energy and scoring volume were determined by weighting the $\bar{y}_{D}$ values of each species by their relative dose contribution. These results were then divided by the $\bar{y}_{D}$ results for the $250 \mathrm{keV}$ x-rays in the corresponding volume to generate a prediction of neutron RBE (Equation 5.1). While propagating the uncertainties, all values were assumed to be independent.

$$
R B E=\frac{\sum_{i} d_{i} \bar{y}_{D, i}}{\bar{y}_{D, x}}
$$

Predicted neutron RBE results were then compared with published radiation weighting factors for neutrons. The ICRP recommends a continuous function of energy described by the piece-wise equation [10]:

$$
w_{R}= \begin{cases}2.5+18.2 e^{-\left[\ln \left(E_{n}\right)\right]^{2} / 6} & E_{n}<1 \mathrm{MeV} \\ 5.0+17.0 e^{-\left[\ln \left(2 E_{n}\right)\right]^{2} / 6} & 1 \mathrm{MeV} \leq E_{n} \leq 50 \mathrm{MeV} \\ 2.5+3.25 e^{-\left[\ln \left(0.04 E_{n}\right)\right]^{2} / 6} & E_{n}>50 \mathrm{MeV}\end{cases}
$$

The RBE predictions are shown for each scoring volume in Fig. 6.9 alongside Equation 6.2 plotted for neutron energies $E_{n} \in\left[1 \times 10^{-6}, 10\right] 10 \mathrm{MeV}$ and US NRC 
data [54] over the same range. For all three scoring volumes, the predicted RBE was relatively low (generally close to 1 ) and constant in the low energy region (below about $100 \mathrm{keV})$. A large peak $\left(\mathrm{RBE}_{\max }\right)$ then forms, reaching a maximum value near $1 \mathrm{MeV}$ before falling back off. With increasing depth, the height of the $\mathrm{RBE}_{\max }$ peak was decreased and shifted to higher energies. All sampling diameters showed roughly the same trend but with varying magnitudes. At low energies, all sampling diameters predict a similar RBE, but the discrepancies became more pronounced at higher neutron energies in the peak region. Here, the smaller the sampling volume the more reduced the peak, with the $2 \mathrm{~nm}$ results barely larger than those in the low energy region. Comparing the data sets with the ICRP graph, the $1000 \mathrm{~nm}$ results provided the closest agreement for all volumes. Taking the maximum $w_{R}$ of 20.7 at $1 \mathrm{MeV}$ as an indicative value, an $\mathrm{RBE}_{\max }$ of $17 \pm 1$ occurring at $1.5 \mathrm{MeV}$ was found for the inner scoring volume. For the intermediate and outer volumes, values of $18 \pm 1$ and $22.0 \pm 0.6$ were found at $0.9 \mathrm{MeV}$ and $0.5 \mathrm{MeV}$, respectively. 


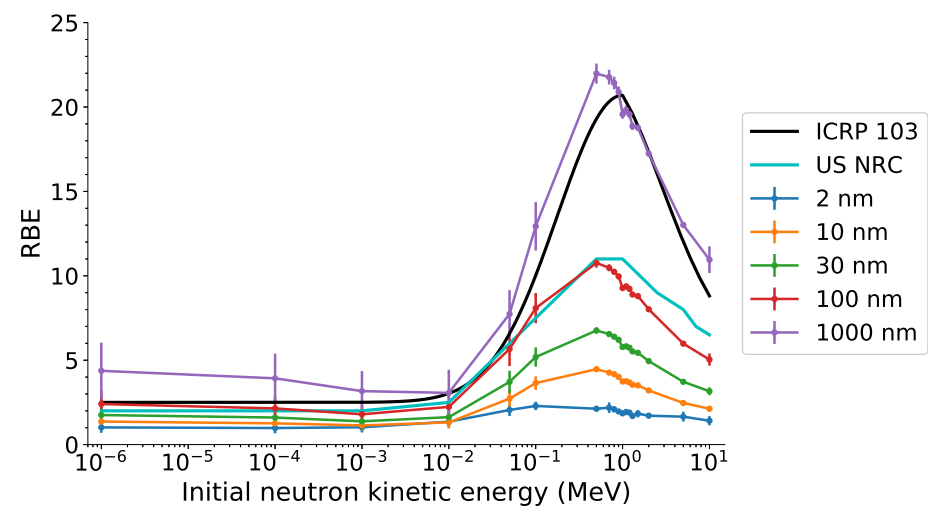

(a)

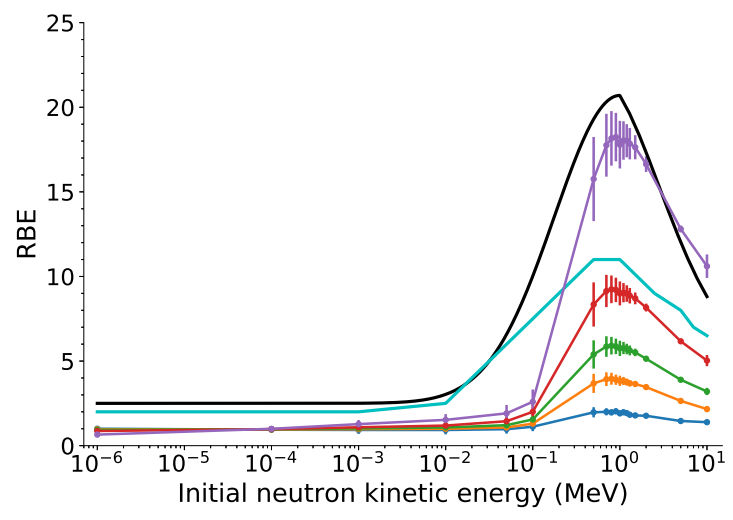

(b)

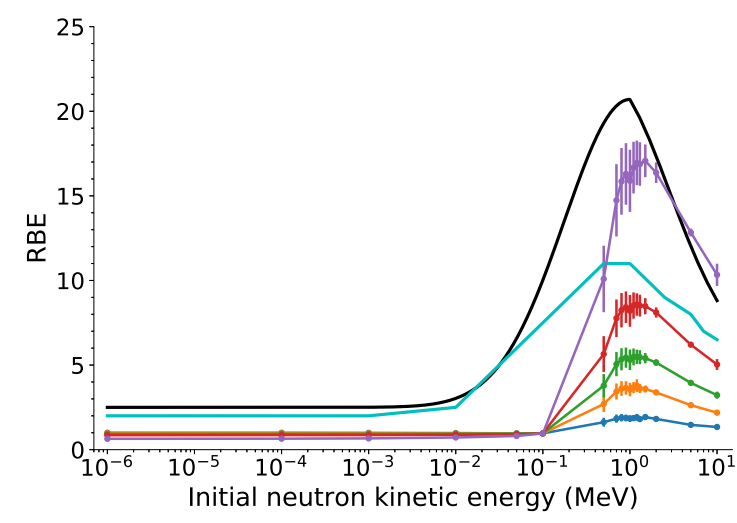

(c)

Figure 6.9: Predicted neutron RBE, as calculated by Equation 6.1, for a range of mono-energetic neutron sources and spherical sampling volume diameters in all three scoring volumes considered. The current ICRP [10] and US NRC [54] radiation weighting factors are plotted for qualitative comparison. Error bars were determined by propagating the inter-run standard deviations of each of the component data sets. Lines are drawn to guide the eye. (a) Outer scoring volume, (b) intermediate scoring volume, (c) inner scoring volume. 



\section{Chapter 7}

\section{Discussion}

In this chapter, a physical rationale for the observed energy dependence of published ICRP and US NRC radiation weighting factors is discussed. A detailed analysis of the competing physics interactions that describe the shape of the secondary and higher order particle spectra and their relative dose contributions is provided. These analyses facilitated explanation of the physics underlying trends in the individual particle species' $\bar{y}_{D}$ and, ultimately, the key neutron RBE for stochastic effects results.

\subsection{Charged particle spectra}

\subsubsection{Neutron-generated spectra}

\section{Secondary particles}

As neutrons traversed the ICRU-4 phantom, their energies were moderated through interactions. Thus, the neutron spectrum was more polyenergetic and had a lower effective energy at increased depths in the medium. This moderation explains the observed skew of heavy ions produced by scattering interactions towards lower energies at increasing depths (Fig. 6.1, left to right) because the energy of the recoil nuclei is dependent on the energy of the incident neutron (Equation 2.8). Above neutron energies of a few $\mathrm{MeV}$ the proportion of protons began to decrease, while the proportion of gammas and heavy ions increased (e.g. from Fig. 6.1(d) to Fig. 6.1(g)). This is a result of the neutron energy overcoming inelastic scattering thresholds of carbon (first level 4.439 MeV), nitrogen (first level $2.311 \mathrm{MeV}$ ), and oxygen nuclei (first level $6.05 \mathrm{MeV}$ ) [106], leading to the production of high energy gammas, recoil nuclei, and smaller fragments such as tritons and alpha particles. At the same time, the elastic cross section for hydrogen decreased and was not compensated for by any inelastic scattering reactions (not possible with ${ }^{1} H$ ).

The observed decrease in secondary heavy ion proportions with increasing depth (Fig. 6.1, left to right) can be explained by considering that neutron capture and direct elastic scattering are the dominant neutron interactions below a few $\mathrm{MeV}$. Direct 
elastic scattering cross sections are essentially constant at these energies, while the capture cross sections increase with decreasing energy (with the exception of some resonances). The net result is that the proportion of capture reactions increases with increasing depth. In human tissue, the most prevalent capture reaction is the ${ }^{1} \mathrm{H}(\mathrm{n}, \gamma){ }^{2} \mathrm{H}$ $(Q=2.225 \mathrm{MeV})$ interaction due to the abundance of hydrogen. Thus, as depth was increased, the proportion of high energy gammas increased relative to heavy ions from scattering interactions. Note that for $10 \mathrm{MeV}$ neutrons (Fig. 6.1, bottom row), the effect appeared less pronounced. This is because relative thermalization was less due to the neutrons' penetrating power.

There were two exceptions to this trend; deuterons and, to a lesser extent, carbon ions. In the former case, deuterons were essentially only produced as the by-products of the hydrogen capture reaction because the natural abundance of the ${ }^{2} \mathrm{H}$ isotope is very low and thus it was negligible as a scattering target. Consequently, the number of deuterons increased with depth. In the latter case, carbon ions were produced by the ${ }^{14} \mathrm{~N}(\mathrm{n}, \mathrm{p}){ }^{14} \mathrm{C}(Q=0.626 \mathrm{MeV})$ neutron capture reaction. Thus the decrease in elastically scattered carbon ions with depth was partially compensated for by an increase in capture recoil carbon ions. As a result, the proportion of carbon ions was decreased to a lesser degree than other heavy ions, with increasing depth. This can be seen from the increase of the $\sim 50 \mathrm{keV}$ peak (capture recoil) as the height of the rest of the carbon spectrum (elastic recoil) was decreased (Fig. 6.1(d)-6.1(f), red curve). While the number of protons was also increased due to capture reactions, they were few in comparison with those protons liberated by direct scattering of neutrons with hydrogen atoms (note the height of the $\sim 580 \mathrm{keV}$ peak relative to the broader elastic spectrum in Fig. 6.1(d)-6.1(f)).

\section{Higher order electron spectra}

The generated electron spectra (Fig. 6.2) have three notable features: (i) low depth and neutron energy dependence, (ii) a peak at $1 \mathrm{MeV}$, and (iii) a peak at $1 \mathrm{keV}$. To explain the first feature, consider that below the inelastic thresholds, all electrons were ultimately generated from the ${ }^{1} \mathrm{H}(\mathrm{n}, \gamma)^{2} \mathrm{H}$ capture reaction. Following the energeticallyfavourable formation of the deuteron nucleus, nearly all of the excess energy is released in the form of a $\sim 2.225 \mathrm{MeV}$ gamma. Although the proportion of gammas to ions changed with energy and depth, the energy of the hydrogen capture gammas is constant. Considering this constancy, one may have predicted that the electron spectra would not change with depth and neutron energy. However, while the gamma spectra was recorded at the point of production, these gammas were then allowed to propagate and interact throughout the volume. Thus, the spectra of gammas seen by the electrons at different depths was potentially subject to large fluctuations. It appears that for the 
ICRU-4 phantom geometry studied here, the variations cancelled each other out. This may not be the case for other geometries and will be interesting to monitor in future work.

The second feature, the $1 \mathrm{MeV}$ peak, was due to the maximum energy allowed by Geant4-DNA for TS simulations. Recall that in the CH simulations, electrons greater than $1 \mathrm{MeV}$ were not killed immediately and recorded in the histogram. Rather, they were permitted to slow down and generate higher order electrons and gammas. Once they were slowed to $\leq 1 \mathrm{MeV}$ they were killed and recorded; hence the observed peaks in Fig. 6.2.

The third feature, the $1 \mathrm{keV}$ peak, is more complex, and arose from the Geant4 models of electron-electron scattering. It is necessary to note that $\mathrm{CH}$ simulations employ multiple scattering theories and high energy approximations. Although it is possible to override lower energy limits in the simulation settings, it may lead to physically inaccurate results. In Geant4, hard electron-electron collisions are governed by an approximation of the standard Møller scattering theory (see [20]) whereby all secondaries must be produced with kinetic energy $E_{K} \gg I$, where $I$ is the mean excitation energy of the medium under study. Below the threshold (default is $1 \mathrm{keV}$ ), electrons are not generated as secondary particles and the energy is instead deposited according to the CSDA approximation. Because the mean excitation energy of the ICRU-4 material is $74.9 \mathrm{eV}$, manually decreasing the energy threshold much below $1 \mathrm{keV}$ would invalidate the $E_{K} \gg I$ approximation. This limitation could possibly reduce the accuracy of electron $\bar{y}_{D}$ results because an evaluation of the $\bar{y}_{D}$ of monoenergetic electrons showed that there are significant variations below $1 \mathrm{keV}$ for all of the sampling diameters studied (Fig. 7.1). Notably, electron $\bar{y}_{D}$ peaks near $1 \mathrm{keV}$ before rapidly declining with increasing energy. In the future it would be useful to develop a code to calculate the theoretical slowing down spectrum of the electrons generated above $1 \mathrm{MeV}$ in order to improve the accuracy of the electron spectra passed to Geant4-DNA simulations. On the other hand, it is expected that electron transport will be extended above $1 \mathrm{MeV}$ in future releases of Geant4-DNA [107], potentially rendering this pursuit unnecessary.

The only noticeable energy or depth dependence exhibited by the electron spectra was seen in the reduction of the $1 \mathrm{MeV}$ peak relative to the $1 \mathrm{keV}$ peak when inelastic scattering became possible (compare Fig. 6.2(d) and 6.2(g)). While Compton scattering was still the dominant process for the higher energy gammas produced during these interactions, the secondary electrons were produced with higher energies due not only to the increased photon energies but also to the higher average and maximum Compton energy transfer fractions (see Fig. 7.2). Consequently, these electrons required a larger number of interactions to be slowed down to $1 \mathrm{MeV}$ and therefore produced more low 


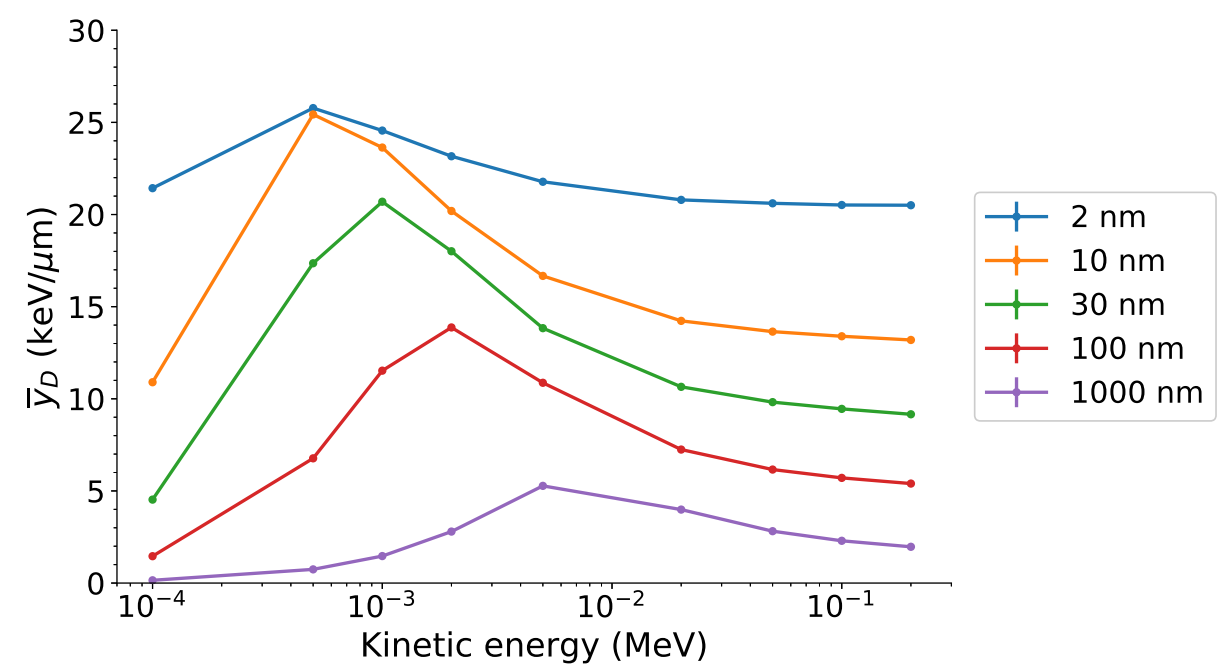

FiguRE 7.1: Dose-mean lineal energy $\left(\bar{y}_{D}\right)$ of mono-energetic electrons calculated for several spherical scoring volume diameters in liquid water using the weighted track-sampling algorithm in Geant4-DNA.

energy delta electrons.

\subsection{2 $250 \mathrm{keV}$ x-ray secondary particle spectra}

Compton scattering is the dominant interaction in human tissue for $250 \mathrm{keV}$ photons [21]. At this energy, the average energy transfer fraction due to a Compton interaction is about 0.25 , and the maximum possible energy transfer is 0.5 (Fig. 7.2). Consequently, a local maximum was seen near $50 \mathrm{keV}$ and a sharp threshold was apparent near $125 \mathrm{keV}$ in the secondary electron spectra for all three scoring volumes (Fig. 6.3). Attenuation led to a lower effective energy as the photons propagated deeper into the tissue.

\subsection{Relative dose contributions}

At low initial neutron energies, the electrons dominated the dose within all volumes (Fig. 6.4) due to the prevalence of the hydrogen capture reaction. The gammas liberated by this reaction proceeded to liberate electrons, which deposit the dose. This was because the number of gammas increased with decreasing neutron energy and they were always produced with a relatively high energy of $\sim 2.225 \mathrm{MeV}$. In contrast, although the cross section for hydrogen elastic scattering is similar to that for hydrogen capture, the energy of the protons produced by such interactions decreases with neutron energy (Equation 2.8). Thus, at lower neutron energies, the electrons produced by the secondary gammas were more prevalent and energetic in comparison with the protons. This was exacerbated by neutron moderation and, as a result, the effect was more pronounced in the deeper scoring volumes (Fig. 6.1, left to right). As neutron energy increased, the 


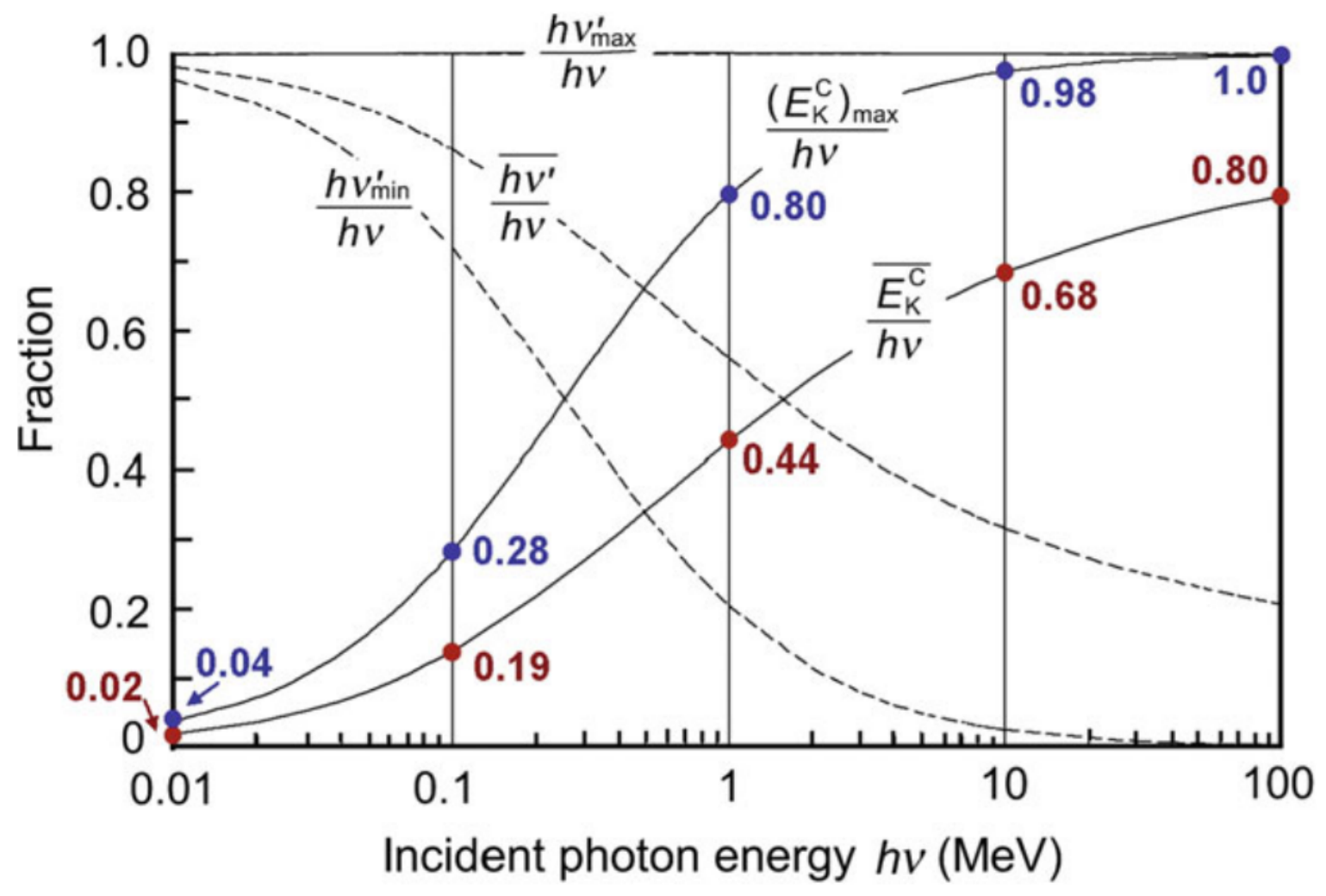

Figure 7.2: The mean and maximum fractions of incident photon energy $h \nu$ transferred to the kinetic energy of the recoil electron $\left(E_{K}^{\mathrm{C}}\right)$ during Compton scattering as a function of $h \nu$. Mean, maximum, and minimum fraction of $h \nu$ retained by the scattered photon $\left(h \nu^{\prime}\right)$ are also shown. Figure from Podgoršak [21]. 
opposite trend was observed: thermalization became less likely, the cross section for neutron capture at higher energies was decreased, and the average energy transferred to protons during elastic scattering interactions increased (Fig. 6.1, top to bottom). Consequently, at high neutron energies, the proton contribution exceeded that of the electrons and dominated the dose. The cross-over point occurred at higher energies for the deeper volumes due to moderation (Fig. 6.4).

Elastic scattering of heavier ions occurred at all energies but the dose contributions were low below the inelastic thresholds (less prominent at increased depths) because the energy transfer fraction of elastic scattering decreases with increasing target mass (Equation 2.8). Above the inelastic thresholds, various inelastic reactions led to a further increase in heavy ion dose contributions. Especially noticeable was the sharp rise of the alpha contribution. Unlike carbon, nitrogen, and oxygen, the major source of alphas below the inelastic thresholds was the weak capture reaction with the uncommon ${ }^{17} \mathrm{O}$ isotope (which also produces carbon) because there is no helium present for their production by elastic scattering.

The results of the explicit dose calculations showed strong agreement with the local approximation for nearly all data points in every volume of the three test cases $(1 \mathrm{eV}$, $1 \mathrm{MeV}$, and $1010 \mathrm{MeV}$ ). However, the local approximation clearly underestimated the electron dose contribution at high energies. The effect was most pronounced for the $10 \mathrm{MeV}$ case and it is believed that this arose because the assumption of CPE did not hold above the inelastic thresholds (a few $\mathrm{MeV}$ ). At these neutron energies, gammas were produced above $3 \mathrm{MeV}$ (Fig. 6.1, bottom row), which has been recommended as the upper photon energy limit for the validity of the CPE assumption by the ICRP [94]. Nevertheless, the local approximation was considered sufficient for the purposes of this thesis, as its shortcomings for fast neutrons represent only a few percent of the total dose (explicitly calculated electron $d_{i}<3 \%$ for $10 \mathrm{MeV}$ neutrons) while they are negligible elsewhere.

\subsection{Dose-mean lineal energies}

This section will explore the physical rationale for the trends observed in Fig. 6.5 for each secondary particle $\bar{y}_{D}$.

The energy dependence of secondary proton $\bar{y}_{D}$ is notable for two features: (i) the presence of a peak near $1 \mathrm{MeV}$ and (ii) the presence of a local minimum between the $1 \mathrm{MeV}$ peak and epithermal energies. Regarding the first trend, the $1 \mathrm{MeV}$ peak can be readily explained by the spectrum of recoil (elastically scattered) protons. To illustrate this, Fig. 7.3 shows the results of a theoretical calculation by Kellerer and Chmelevsky [108] of the $\bar{y}_{D}$ of recoil proton spectra produced by mono-energetic neutrons in liquid 


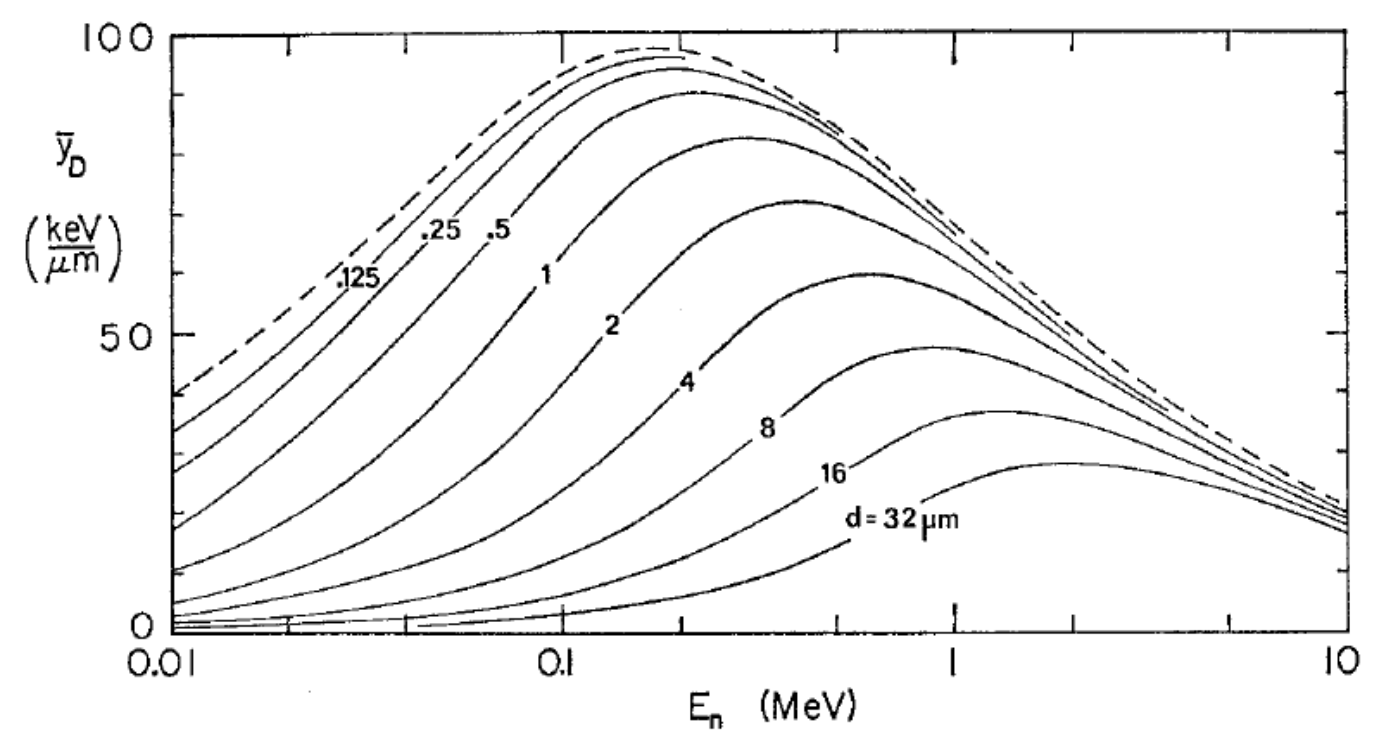

FiguRE 7.3: Theoretically calculated $\bar{y}_{D}$ of the spectrum of protons generated by neutron elastic scattering with hydrogen nuclei in liquid water. Data is presented for multiple scoring sphere diameters for monoenergetic neutrons with initial kinetic energies between $10 \mathrm{keV}$ and $10 \mathrm{MeV}$. Figure from Kellerer and Chmelevsky [108].

water for a variety of sampling diameters. The shapes of their results over the upper intermediate and fast neutron energy range for volumes with diameters $\leq 1 \mu \mathrm{m}$ are quite similar to the trends observed in Fig. 6.5 (blue curve).

The presence of the minimum near $\sim 10 \mathrm{keV}$ and the subsequent increase in $\bar{y}_{D}$ with decreasing energy ( Fig. 6.5(a), blue curve) closely match a similar trend in the nitrogen capture $\left({ }^{14} \mathrm{~N}(\mathrm{n}, \mathrm{p}){ }^{14} \mathrm{C}\right.$ ) cross section (Fig. 7.4). Furthermore, the $\sim 580 \mathrm{keV}$ protons produced by this reaction coincide with a maximum in proton $\bar{y}_{D}$ (compared to other mono-energetic protons). Thus, it appears that the observed rise in the $\bar{y}_{D}$ of the overall proton spectra with decreasing energy was due to an increase in the proportion of nitrogen capture protons. This was further borne out by a less prominent dip in the observed $\bar{y}_{D}$ with depth, as would be expected for a capture reaction effect. A possible counter-argument is that the proportion and dose contribution of capture protons was small in comparison with the proportion of recoil protons, as discussed in Section 7.1.1. However, this may have been compensated for by the fact that the associated volume calculation assigned a larger weight to capture protons than recoil protons due to the former's higher energy and thus range. The relatively high value of $\bar{y}_{D}$ at low neutron energies was also reported by Baiocco et al. [63] but no $\bar{y}_{D}$ minimum was observed in their data for intermediate neutrons. It is likely that this lack of agreement is due to differences between the explicit generation of tracks (this work) and the use of the PHITS microdosimetric function (Baiocco et al. [63]).

The $\bar{y}_{D}$ values of the electron spectra were essentially constant below the inelastic 


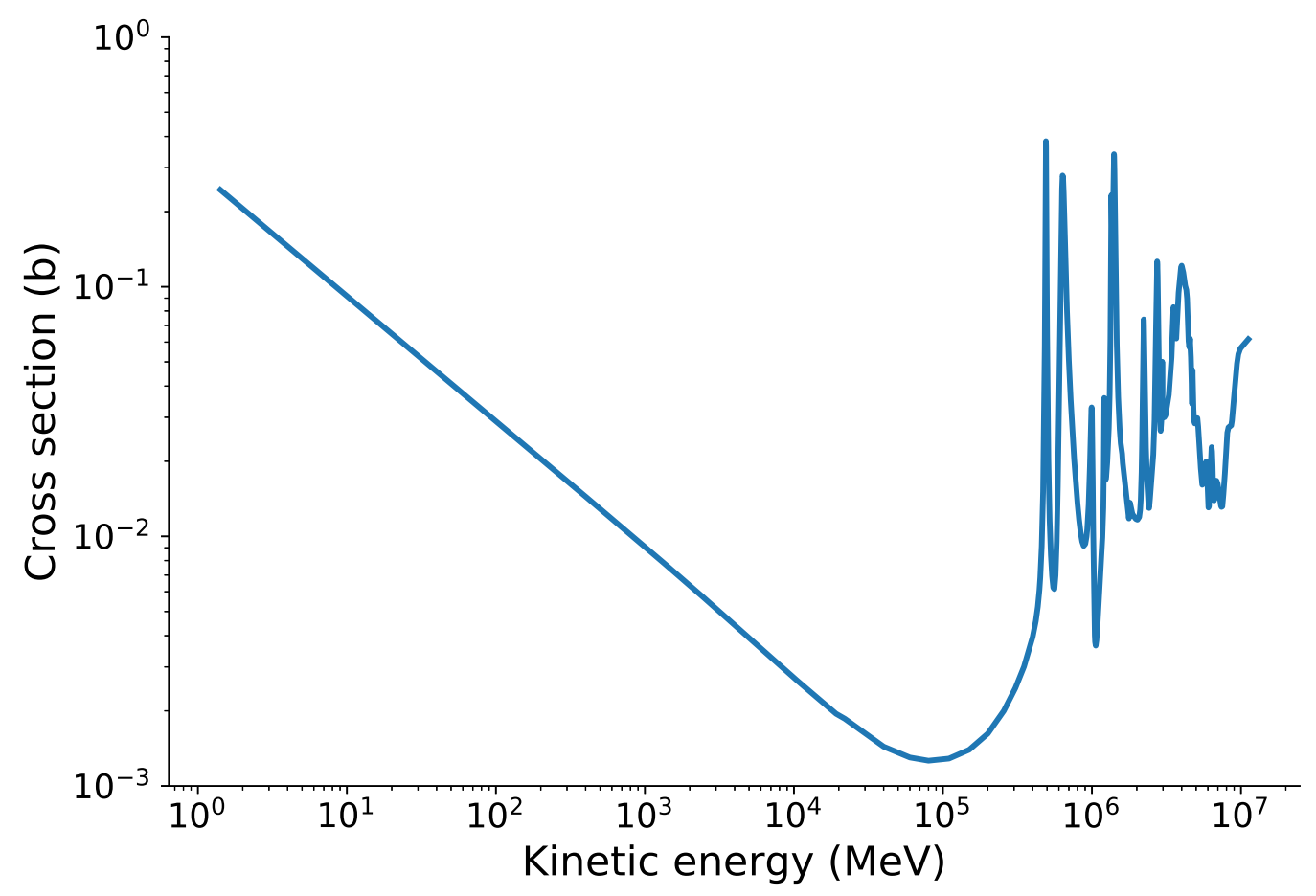

Figure 7.4: Cross section $\left(b=10^{-24} \mathrm{~cm}^{2}\right)$ of the ${ }^{14} \mathrm{~N}(\mathrm{n}, \mathrm{p}){ }^{14} \mathrm{C}$ reaction vs. neutron energy. Data from ENDF/B-VII.1 [90].

thresholds (Fig. 6.5, orange curve) because of the negligible energy and depth dependence of the electron spectra (Section 7.1.1). However, as can be seen in Fig. 6.7, the $\bar{y}_{D}$ of the electron spectra increased as neutron energy was increased from $1 \mathrm{MeV}$ to $10 \mathrm{MeV}$. To explain this, recall that for neutron energies above the inelastic thresholds, the lower energy component of the electron spectra was seen to increase at the expense of the $1 \mathrm{MeV}$ peaks (Fig. 6.2(d) and 6.2(g)). From Fig. 7.1, decreasing the electron energy generally resulted in an increase in electron $\bar{y}_{D}$. Hence, as electron spectra became weighted towards lower energies with increasing neutron energy, the $\bar{y}_{D}$ of these spectra increased.

For all depths, the $\bar{y}_{D}$ of the alpha spectra decreased from those produced by $5 \mathrm{MeV}$ neutrons to those produced by $10 \mathrm{MeV}$ neutrons (Fig. 6.5, green). Baiocco et al. [63] explained this trend by noting that at higher neutron energies, more energy is available to the secondary alphas during inelastic reactions and thus they produce less densely ionizing tracks. A comparison of alpha spectra shown in Fig. 7.5 provides evidence to support this claim.

\subsection{Neutron RBE}

Building upon the results discussed so far, this section presents a physical rationale for the energy dependence of neutron RBE. In particular, two trends in the 


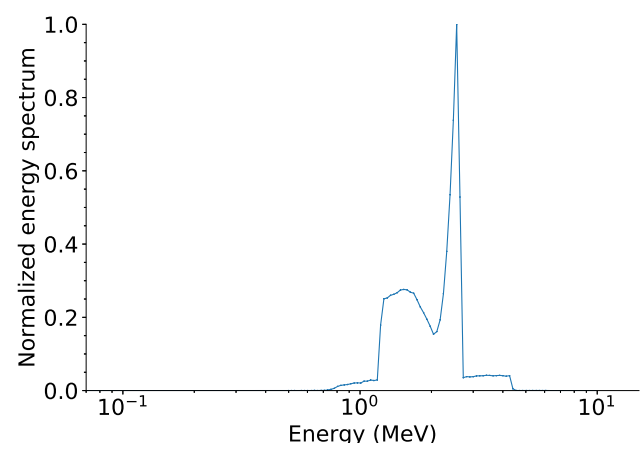

(a)

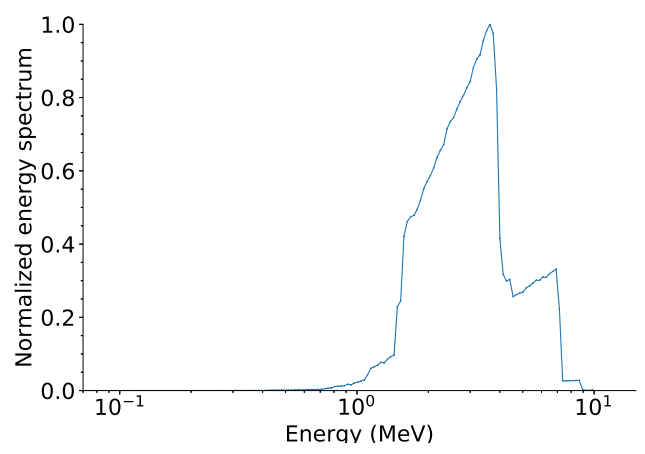

(b)

Figure 7.5: The spectra of alpha particles produced in the outer scoring volume by mono-energetic neutron sources with initial kinetic energies of (a) $5 \mathrm{MeV}$ and (b) $10 \mathrm{MeV}$. Spectra are normalized to the largest value.

microdosimetrically-predicted RBE results are highlighted and explained in the context of the underlying neutron interactions.

First, it can be seen in Fig. 6.9 that for neutron energies in the intermediate range and below $(\leq 50 \mathrm{keV})$, neutron RBE was low $(\lesssim 3)$ and approximately constant for virtually all sampling diameters in each scoring volume. This trend arose from the predominance of the hydrogen capture reaction at low energies. Although capture reactions are most probable at thermal energies $(\approx 0.025 \mathrm{eV})$, thermalisation of the neutrons as they traversed the ICRU-4 sphere resulted in a significant number of such interactions for non-thermal initial energies, especially at larger depths. Due to the high energy of the resulting gammas, which can be seen in the peaks near $2.225 \mathrm{MeV}$ in Figure 6.1, the dose they deposited exceeded the dose deposited during the thermalisation process. Therefore, the hydrogen capture reaction led to the majority of the neutron dose being deposited by nearly identical electron spectra for low neutron energies (Figures 6.2 and 6.4). As the dose from the x-ray reference radiation was also deposited by electrons, the neutron and x-ray fields were microdosimetrically similar. Indeed, they agreed almost exactly for all sampling diameters. Every increase in neutron-generated electron $\bar{y}_{D}$ with decreasing sampling diameter (Fig. 6.7) was balanced by a corresponding increase in the x-ray electron spectra $\bar{y}_{D}$ (Fig. 6.8). The net result was that neutron RBE was relatively low and independent of both neutron energy and sampling diameter for all scoring volumes at low energies. The results were in qualitative agreement with the ICRP and US NRC radiation weighting factors, both of which recommend a low, constant RBE at these energies. The agreement is strongest in the outer volume, where elastically scattered protons result in slightly higher predicted RBE values than in the other volumes. 
The second trend in the neutron RBE graph was the prominent peak centred at energies near $1 \mathrm{MeV}$ (Fig. 6.9). As the proton dose contribution began to dominate over the electron contribution, the significantly larger $\bar{y}_{D}$ of the proton spectra (see Figure 6.5 for example) was reflected in an increase in predicted neutron RBE. The peak coincided with the maximum in the proton $\bar{y}_{D}$ results for each sampling diameter (compare to Fig. 6.6) and thus arose from the recoil protons. In contrast to the electrons, the effect of sampling diameter on proton $\bar{y}_{D}$ was far less pronounced (Fig. 6.6). Consequently, the relative difference between proton and electron $\bar{y}_{D}$ increases with increasing sampling diameter. This can be seen in Fig. 6.9 from the increase in RBE max and the steepness of the rise towards it for larger sampling diameters.

Strong qualitative agreement was seen with both the ICRP and the US NRC radiation weighting factors. The ICRP recommend a sharper and more prominent $1 \mathrm{MeV}$ peak than do the US NRC and thus the shape of the ICRP graph was better described by a larger sampling volume. Namely, the ICRP radiation weighting factors most closely resemble the $1000 \mathrm{~nm}$ sampling diameter results, while the US NRC radiation weighting factors were best described by the $100 \mathrm{~nm}$ sampling diameter results. The fact that the two sets of radiation weighting factors were best described by different sampling diameters highlights an important point about microdosimetry: due to the spatial dependence of the biological consequences of radiation-induced DNA damage, no single sampling volume size can predict all biological effects. However, it should be stressed that the values presented here should be understood only as a means of providing confidence in the trends identified in the constituent data sets (spectra, doses, and $\bar{y}_{D}$ 's), rather than as a quantitative assessment of true neutron RBE. In this regard, the results presented here are in agreement with explanations of the energy dependence of neutron RBE [63] and secondary dose contributions [12]. 


\section{Chapter 8}

\section{Summary and Conclusions}

During high-energy photon radiation therapy, patients are subjected to a whole-body neutron dose originating from photonuclear reactions that take place throughout the treatment room and within the patient. Although this dose may be far lower than that needed for appreciable cell killing, radiation carcinogenesis is thought to be a stochastic effect, meaning that there is no dose threshold below which the induction of cancer cannot occur. The nature of the interplay between the complexities of ionizing radiation interactions with human tissue and the response of biological systems is not fully understood. However, microdosimetry allows for the indirect study of carcinogenesis by linking the initial microscopic energy deposition pattern with observed biological effects. In the case of the neutron field experienced by radiation therapy patients, the microdosimetric quantity dose-mean lineal energy $\bar{y}_{D}$ can be used for such an investigation. This thesis investigated the energy and depth dependence of neutron RBE for carcinogenesis by comparing the $\bar{y}_{D}$ of mono-energetic neutron fields incident on a tissue phantom to that of $250 \mathrm{keV}$ x-rays for a number of sampling volume diameters. The results were obtained via a simulation pipeline that combined $\mathrm{CH}$ simulations with a TS code featuring a weighted track-sampling algorithm that operated on charged particle spectra and corrected for biases towards smaller tracks and regions denser in transfer points.

Qualitative agreement was found with the experimentally and epidemiologically derived weighting factors of the ICRP and US NRC for all depths and sampling volume diameters. It was shown that the low, flat portion of the weighting factor graphs below $100 \mathrm{keV}$ resulted from the high cross section and energy transfer of the hydrogen capture reaction, while the peak near $1 \mathrm{MeV}$ was primarily the result of neutronhydrogen direct elastic scattering. Decreasing sampling volume diameter tended to decrease the $\bar{y}_{D}$ for each particle and had an especially marked effect on the RBE peak. For all sampling volumes, RBE was larger at shallower depths due to a reduced likelihood of thermalization and thus a higher proportion of secondary ions relative to secondary gammas. The strongest agreement was found with the ICRP factors for $1000 \mathrm{~nm}$ sampling volumes in the outer scoring volume, while the strongest agreement 
with the NRC factors was found for $100 \mathrm{~nm}$ sampling volumes in the outer scoring volume. A quantitative comparison with the final results of Baiocco et al. [63] could not be performed due to the lack of low energy $(<0.5 \mathrm{MeV})$ heavy ion transport in Geant4-DNA, but overall the trends were largely in agreement.

Neutron microdosimetry in Geant4-DNA is currently limited by the energy range of TS models for heavy ions. This issue will require a great deal of attention and effort in the future. However, the ability to predict biological trends by studying only protons, electrons, and alphas shows that Geant4-DNA is already sophisticated enough for many studies. For example, microdosimetric results may be compared with other related methods. Villegas et al. [18] describe a clustering algorithm in which energy deposition patterns are not restricted to any single volume, but are allowed to form clusters of arbitrary size and shape as long as each transfer point is always within a pre-defined distance of a neighbour. It would be instructive to study how the frequency of cluster sizes, unweighted by energy and unrestricted by geometrical bounds, would fare in predicting biological damage relative to lineal energy for neutrons. A version of this algorithm has already been implemented within Geant4-DNA by the author. A multi-scale MC approach may also be taken in the vein of a recent publication by Zabihi et al. [88]. These techniques seek to explicitly model not just the physical interactions of radiation, but also the subsequent physicochemical and chemical stages in order to predict DNA damage. They often feature a geometric model of DNA and, more recently, some groups have even begun to develop probabilistic models of DNA repair pathways $[109,110]$.

With regards to the current pipeline, there are several avenues of investigation left open. A systematic study of the effects of phantom and scoring volume geometries should be performed and improvements to the electron slowing down method should be considered. The relevance of protons from the nitrogen capture reactions to the $\bar{y}_{D}$ trends discussed in Section 7.3 may also be tested by considering their relative dose and lineal energy independently from those of the scattered protons. It would also be of interest to test the extent to which heavy ions impact the overall saturation-corrected dose-mean lineal energy $\left(y^{*}\right)$ by comparing values calculated with Geant4-DNA to those published by Baiocco et al. [63]. Towards this end, the author has adapted the sampling algorithm according to a discretization of Eq. 4.15.

As a standalone tool, the weighted track-sampling algorithm implementation may find uses in other microdosimetric applications. It can be readily adapted for the calculation of other quantities or the recording of full distributions. Improvement efforts should focus on an implementation of the general solution to the calculation of the union of an arbitrary set of spheres described by Cazals et al. [111]. Results between the approximation and the general solution could be compared to test the 
quality of the approximation against the speed improvement it may confer. 



\section{Bibliography}

${ }^{1}$ Canadian Cancer Society, Canadian Cancer Statistics, tech. rep. (2018).

${ }^{2}$ W. Newhauser, and M. Durante, "Assessing the risk of second malignancies after modern radiotherapy", Nature Reviews Cancer 11, 438 (2011).

${ }^{3}$ IAEA, Radiation Oncology Physics (IAEA, Vienna, 2005).

${ }^{4}$ H. Patrick, "Development of a DVH Registry for Plan Evaluation, Dose Accumulation, and Cohort Analysis" (McGill University, Montreal, Canada, 2018).

${ }^{5}$ F. Khan, The Physics of Radiation Therapy, $5^{\text {th }}$ Ed. (Lippincott Williams \& Wilkins, Philadelphia, Pa., 2014).

${ }^{6}$ D. Eaton, "Electronic brachytherapy - current status and future directions", The British Journal of Radiology 88, 20150002 (2015).

${ }^{7}$ H. Owen, A. Lomax, and S. Jolly, "Current and future accelerator technologies for charged particle therapy", Nuclear Instruments and Methods in Physics Research Section A 809, 96-104 (2016).

${ }^{8}$ P. Anand, A. Kunnumakara, C. Sundaram, K. Harikumar, S. Tharakan, O. Lai, B. Sung, and B. Aggarwal, "Cancer is a preventable disease that requires major lifestyle changes", Pharmaceutical Research 25, 2097-2116 (2008).

${ }^{9}$ E. Hall, and A. Giaccia, Radiobiology for the Radiologist, $7^{\text {th }}$ Ed. (Lippincott Williams \& Wilkins, Philadelphia, Pa., 2012).

${ }^{10}$ ICRP, "The 2007 Recommendations of the International Commission on Radiological Protection. ICRP Publication 103", Annals of the ICRP 37 (2007).

${ }^{11}$ J. Little, "Radiation carcinogenesis", Carcinogenesis 21, 397-404 (2000).

${ }^{12}$ D. Satoh, F. Takahashi, A. Endo, Y. Ohmachi, and N. Miyahara, "Calculation of Dose Contributions of Electron and Charged Heavy Particles inside Phantoms Irradiated by Monoenergetic Neutron", Journal of Radiation Research 49 (2008).

${ }^{13}$ K. Moore, The Radium Girls: The Dark Story of America's Shining Women (Sourcebooks, Naperville, IL, 2017).

${ }^{14}$ E. Cardis, M. Vrijheid, M. Blettner, E. Gilbert, M. Hakama, C. Hill, G. Howe, J. Kaldor, and C. Muirhead, "Risk of cancer after low doses of ionising radiation: retrospective cohort study in 15 countries", The BMJ 331 (2005). 
${ }^{15}$ S. Incerti, M. Douglass, S. Penfold, S. Guatelli, and E. Bezak, "Review of Geant4DNA applications for micro and nanoscale simulations", Physica Medica 32, 11871200 (2016).

${ }^{16}$ M. Bernal, M. Bordage, J Brown, M. Davidková, E. Delage, Z. El Bitar, S. Enger, Z. Francis, S. Guatelli, V Ivanchenko, et al., "Track structure modeling in liquid water: a review of the Geant4-DNA very low energy extension of the Geant4 Monte Carlo simulation toolkit", Physica Medica 31, 861-874 (2015).

${ }^{17}$ D. Goodhead, "Initial events in the cellular effects of ionizing radiations: clustered damage in DNA", International Journal of Radiation Biology 65, 7-17 (1994).

${ }^{18}$ F. Villegas, G. Bäckström, N. Tilly, and A. Ahnesjö, "Energy deposition clustering as a functional radiation quality descriptor for modeling relative biological effectiveness", Medical Physics 43, 6322-6335 (2016).

${ }^{19}$ N. Lampe, M. Karamitros, V. Breton, J Brown, D. Sakata, D. Sarramia, and S. Incerti, "Mechanistic DNA damage simulations in Geant4-DNA part 2: electron and proton damage in a bacterial cell", Physica Medica 48, 146-155 (2018).

${ }^{20}$ P. Andreo, D Burns, A Nahum, J. Seuntjens, and F Attix, Fundamentals of Ionizing Radiation Dosimetry (2017).

${ }^{21}$ E Podgoršak, Radiation physics for medical physicists, $3^{\text {rd }}$ Ed. (Springer International Publishing, Switzerland, 2016).

${ }^{22}$ ICRU, Fundamental Quantities and Units for Ionizing Radiation, tech. rep. 85a (ICRU, 2011).

${ }^{23}$ L. Lindborg, and H. Nikjoo, "Microdosimetry and radiation quality determinations in radiation protection and radiation therapy", Radiation Protection Dosimetry 143, 402-408 (2011).

${ }^{24}$ A. Yue, M. Dewey, D. Gilliam, G. Greene, A. Laptev, J. Nico, W. Snow, and F. Wietfeldt, "Improved Determination of the Neutron Lifetime", Physical Review Letters 111 (2013).

${ }^{25}$ K Beckurts, and K. Wirtz, Neutron Physics (Springer-Verlag, 1964).

${ }^{26}$ E. Hussein, Radiation Mechanics: Principles and Practice (Elsevier Science, 2007).

${ }^{27}$ A. Nahum, "Condensed-history monte-carlo simulation for charged particles: what can it do for us?", Radiation and Environmental Biophysics 38, 163-173 (1999).

${ }^{28}$ I. Kyriakou, V. Ivanchenko, D. Sakata, M. Bordage, S. Guatelli, S. Incerti, and D. Emfietzoglou, "Influence of track structure and condensed history physics models of Geant4 to nanoscale electron transport in liquid water", Physica Medica 58, 149-154 (2019). 
${ }^{29}$ I. Chetty, B. Curran, J. Cygler, J. DeMarco, G. Ezzell, B. Faddegon, I. Kawrakow, P. Keall, H. Liu, C.-M. C. Ma, et al., "Report of the aapm task group no. 105: issues associated with clinical implementation of monte carlo-based photon and electron external beam treatment planning", Medical Physics 34, 4818-4853 (2007).

${ }^{30}$ F. Salvat, J. Fernández-Varea, J. Sempau, and J. Mazurier, "Practical aspects of monte carlo simulation of charged particle transport: mixed algorithms and variance reduction techniques", Radiation and Environmental Biophysics 38, 15-22 (1999).

${ }^{31}$ X. Dong, G. Cooperman, J. Apostolakis, S. Jarp, A. Nowak, M. Asai, and D. Brandt, "Creating and improving multi-threaded geant4", in Journal of physics: conference series, Vol. 396, 5 (IOP Publishing, 2012), p. 052029.

${ }^{32}$ S. Agostinelli, J. Allison, K. Amako, J. Apostolakis, H. Araujo, P. Arce, M. Asai, D. Axen, S. Banerjee, G. Barrand, et al., "Geant4-a simulation toolkit", Nuclear Instruments and Methods in Physics Research Section A 506, 250-303 (2003).

${ }^{33}$ J. Allison, K. Amako, J. Apostolakis, H. Araujo, P. Arce Dubois, M. Asai, G. Barrand, R. Capra, S. Chauvie, R. Chytracek, et al., "Geant4 developments and applications", IEEE Transactions on Nuclear Science 53, 270-278 (2006).

${ }^{34}$ J. Allison, K. Amako, J. Apostolakis, P. Arce, M. Asai, T. Aso, E. Bagli, A. Bagulya, S. Banerjee, G. Barrand, et al., "Recent developments in GEANT4", Nuclear Instruments and Methods in Physics Research Section A 835, 186-225 (2016).

${ }^{35}$ S. Incerti, M. Karamitros, A. Mantero, P. Moretto, H Tran, B. Mascialino, C. Champion, V Ivanchenko, M Bernal, Z. Francis, C. Villagrasa, G. Baldacchino, P. Guèye, R. Capra, P. Nieminen, and C. Zacharatou, "Comparison of GEANT4 very low energy cross section models with experimetnal data in water", Medical Physics 37, 4692-4708 (2010).

${ }^{36}$ S. Incerti, I. Kyriakou, M. Bernal, M. Bordage, Z. Francis, S. Guatelli, V. Ivanchenko, M. Karamitros, N. Lampe, S. Lee, S. Meylan, C. Min, W. Shin, P. Nieminen, D. Sakata, N. Tang, C. Villagrasa, H. Tran, and J. Brown, "Geant4-DNA example applications for track structure simulations in liquid water: A report from the Geant4-DNA Project", Medical Physics 45 (2018).

${ }^{37}$ S. Incerti, G. Baldacchino, M. Bernal, R. Capra, C. Champion, Z. Francis, P. Guèye, A. Mantero, B. Mascialino, P. Moretto, P. Nieminen, C. Villagrasa, and C. Zacharatou, "The Geant4-DNA project", International Journal of Modeling, Simulation, and Scientific Computing 1, 157-178 (2010). 
${ }^{38}$ M. Karamitros, A. Mantero, S. Incerti, W. Friedland, G. Baldacchino, P. Barberet, M. Bernal, R. Capra, C. Champion, and Z. El Bitar, "Modeling radiation chemistry in the Geant4 toolkit", Progress in Nuclear Science and Technology 2, 503-508 (2011).

${ }^{39}$ M. Karamitros, S. Luan, M. Bernal, J. Allison, G. Baldacchino, M. Davidkova, Z. Francis, W. Friedland, V. Ivantchenko, A. Ivantchenko, et al., "Diffusion-controlled reactions modeling in geant4-dna", Journal of Computational Physics 274, 841-882 (2014).

${ }^{40} \mathrm{~S}$. Lehnert, Biomolecular action of ionizing radiation, $1^{\text {st }}$ Ed. (CRC Press, Boca Raton, Fl., 2007).

${ }^{41}$ T. Pollard, W. Earnshaw, J. Lippincott-Schwartz, and G. Johnson, Cell Biology, $3^{\text {rd }}$ Ed. (Elsevier Health Sciences, Philadelphia, Pa., 2017).

${ }^{42}$ G. Elgar, and T. Vavouri, "Tuning in to the signals: noncoding sequence conservation in vertebrate genomes", Trends in Genetics 24, 344-352 (2008).

${ }^{43} \mathrm{D}$. Nicholl, An introduction to genetic engineering, $3^{\text {rd }}$ Ed. (Cambridge University Press, New York, NY, 2008).

${ }^{44}$ A. Georgakilas, P. O'Neill, and R. Stewart, "Induction and repair of clustered DNA lesions: what do we know so far?", Radiation Research 180, 100-109 (2013).

${ }^{45}$ B. Michael, and P. O'Neill, "A sting in the tail of electron tracks", Science 287, 1603-1604 (2000).

${ }^{46}$ P. Bradshaw, D. Stavropoulos, and M. Meyn, "Human telomeric protein TRF2 associates with genomic double-strand breaks as an early response to DNA damage", Nature Genetics 37, 193 (2005).

${ }^{47}$ N. Y. Tretyakova, A. Groehler IV, and S. Ji, "DNA-protein cross-links: formation, structural identities, and biological outcomes", Accounts of Chemical Research 48, 1631-1644 (2015).

${ }^{48} \mathrm{~K}$. Magnander, and K. Elmroth, "Biological consequences of formation and repair of complex DNA damage", Cancer Letters 327, 90-96 (2012).

${ }^{49}$ A. Kellerer, "Considerations on the Random Traversal of Convex Bodies and Solutions for General Cylinders", Radiation Research 47, 359-376 (1971).

${ }^{50} \mathrm{G}$. Hartmann, and P. Andreo, Fluence calculation methods in Monte Carlo dosimetry simulations, 2018, in press, corrected proof.

${ }^{51}$ ICRU, Fundamental quantities and units for ionising radiation (revised), tech. rep. 11.1 (ICRU, 2011). 
${ }^{52}$ L. Lindborg, and A. Waker, Microdosimetry: Experimental Methods and Applications (CRC Press, Taylor and Francis Group, Boca Raton, Fl., 2017).

${ }^{53}$ H. Engels, H. G. Menzel, P. Pihet, and A. Wambersie, "Risk assessment for cancer induction after low-and high-LET therapeutic irradiation", Strahlentherapie und Onkologie 175, 47-51 (1999).

${ }^{54}$ NRC Regulations Title 10, Code of Federal Regulations: 10 CFR. §20.1004, 2014.

${ }^{55}$ ICRU, Microdosimetry, tech. rep. 36 (ICRU, 1983).

${ }^{56}$ G. Famulari, P. Pater, and S. Enger, "Microdosimetry calculations for monoenergetic electrons using Geant4-DNA combined with a weighted track sampling algorithm", Physics in Medicine and Biology 62 (2017).

${ }^{57}$ H. H. Rossi, and M. Zaider, "Elements of microdosimetry", Medical Physics 18, 1085-1092 (1991).

${ }^{58}$ A. Kellerer, "Fundamentals of Microdosimetry", in The Dosimetry of Ionizing Radiation, Vol. 1, edited by K. Kase, B. Bjärngard, and F. Attix, (Academic Press London, 1985), pp. 77-162.

${ }^{59}$ H. Nikjoo, and L. Lindborg, "Rbe of low energy electrons and photons", Physics in Medicine and Biology 55, R65-R109 (2010).

${ }^{60}$ J. Lillhök, J.-E. Grindborg, L. Lindborg, I. Gudowska, G. Alm Carlsson, J. Söderberg, M. Kopeć, and J. Medin, "Nanodosimetry in a clinical neutron therapy beam using the variance-covariance method and Monte Carlo simulations", Physics in Medicine and Biology 52, 4953-4966 (2007).

${ }^{61}$ M. Hultqvist, J.-E. Lillhök, L. Lindborg, I. Gudowska, and H. Nikjoo, "Nanodosimetry in a $12 \mathrm{C}$ ion beam using Monte Carlo simulations", Radiation Measurements 45, 1238-1241 (2010).

${ }^{62}$ T. Liamsuwan, M. Hultqvist, L. Lindborg, S. Uehara, and H. Nikjoo, "Microdosimetry of proton and carbon ions", Medical Physics 41, 08172-1-12 (2014).

${ }^{63}$ G. Baiocco, S. Barbieri, G. Babini, J. Morini, D. Alloni, W. Friedland, P. Kundrát, E. Schmitt, M. Puchalska, L. Sihver, and A. Ottolenghi, "The origin of neutron biological effectiveness as a function of energy", Scientific Reports 6 (2016).

${ }^{64}$ G. Famulari, P. Pater, and S Enger, "Microdosimetric Evaluation of Current and Alternative Brachytherapy Sources - a Geant4-DNA Simulation Study", International Journal of Radiation Oncology* Biology* Physics 100, 270-277 (2018).

${ }^{65}$ L. Lindborg, M. Hultqvist, A. Carlsson Tedgren, and H. Nikjoo, "Lineal energy and radiation quality in radiation therapy: model calculations and comparison with experiment", Physics in Medicine and Biology 58, 3089-3105 (2013). 
${ }^{66}$ A. Kellerer, and D. Chmelevsky, "Concepts of microdosimetry. II. Probability distributions of the microdosimetric variables", Radiation and Environmental Biophysicscs 12, 205-216 (1975).

${ }^{67}$ H. H. Rossi, and M. Zaider, Microdosimetry and Its Applications (Springer-Verlag, Berlin, 1996).

${ }^{68}$ D. Chmelevsky, and A Kellerer, "Computation of microdosimetric distributions for small sites", Radiation and Environmental Biophysics 14, 123-136 (1977).

${ }^{69}$ C. Wuu, P. Kliauga, and H. Amols, "Microdosimetric evaluation of relative biological effectiveness for 103-Pd, 125-I, 241-Am, and 192-Ir brachytherapy sources", International Journal of Radiation Oncology* Biology* Physics 36, 689-697 (1996).

${ }^{70}$ P. Pihet, H. Menzel, R. Schmidt, M. Beauduin, and A. Wambersie, "Biological weighting function for RBE specification of neutron therapy beams. Intercomparison of 9 European centres", Radiation Protection Dosimetry 31, 437-442 (1990).

${ }^{71}$ H. G. Menzel, A. Wambersie, and P. Pihet, "The clinical RBE and microdosimetric characterization of radiation quality in fast neutron therapy", Acta Oncologica 33, 251-259 (1994).

${ }^{72}$ J.-P. Pignol, J. Slabbert, and P. Binns, "Monte Carlo simulation of fast neutron spectra: Mean lineal energy estimation with an effectiveness function and correlation to RBE", International Journal of Radiation Oncology* Biology* Physics 49, 251-260 (2001).

${ }^{73}$ H. Paganetti, P. Olko, H. Kobus, R. Becker, T. Schmitz, M. P. Waligorski, D. Filges, and H.-W. Müller-Gärtner, "Calculation of relative biological effectiveness for proton beams using biological weighting functions", International Journal of Radiation Oncology* Biology* Physics 37, 719-729 (1997).

${ }^{74}$ R. Gerlach, H. Roos, and A. Kellerer, "Heavy ion RBE and microdosimetric spectra", Radiation Protection Dosimetry 99, 413-418 (2002).

${ }^{75}$ M. Zaider, and D. J. Brenner, "On the microdosimetric definition of quality factors", Radiation Research 103, 302-316 (1985).

${ }^{76}$ L. Lindborg, and J.-E. Grindborg, "Nanodosimetric results and radiotherapy beams: a clinical application?", Radiation Protection Dosimetry 70, 541-546 (1997).

${ }^{77}$ M. Zaider, and M. N. Varma, "Carcinogenic risk coefficients at environmental levels of radon exposures: a microdosimetric approach.", Health Phys. 70, 837-844 (1996).

${ }^{78}$ A. Kellerer, and H. Rossi, "The theory of dual radiation action", Current Topics in Radiation Research Quarterly VIII, 85-158 (1974). 
${ }^{79}$ H. H. Rossi, and M. Zaider, "Compound dual radiation action: I. General aspects", Radiation Research 132, 178-183 (1992).

${ }^{80}$ A. Wambersie, H. Menzel, R. Gahbauer, D. Jones, B. Michael, and H. Paretzke, "Biological weighting of absorbed dose in radiation therapy", Radiation Protection Dosimetry 99, 445-452 (2002).

${ }^{81}$ A. Ottolenghi, V. Smyth, and K. Trott, "Assessment of cancer risk from neutron exposure-The ANDANTE project", Radiation Measurements 57, 68-73 (2013).

${ }^{82}$ D. Juerß, M. Zwar, U. Giesen, R. Nolte, S. Kriesen, G. Baiocco, M. Puchalska, M-J van Goethem, K. Manda, and G. Hildebrandt, "Comparative study of the effects of different radiation qualities on normal human breast cells", Journal of Radiation Oncology 12 (2017).

${ }^{83}$ A. Ottolenghi, G. Baiocco, V Smyth, K. Trott, and on behalf of the ANDANTE Consortium, "The ANDANTE project: a multidisciplinary approach to neutron RBE", Radiation Protection Dosimetry 166, 311-315 (2015).

${ }^{84}$ T. Sato, K. Niita, N. Matsuda, S. Hashimoto, Y. Iwamoto, S. Noda, T. Ogawa, H. Iwase, H. Nakashima, T. Fukahori, et al., "Particle and heavy ion transport code system, PHITS, version 2.52", Journal of Nuclear Science and Technology 50, 913-923 (2013).

${ }^{85}$ T. Sato, R. Watanabe, L. Sihver, and K. Niita, "Applications of the microdosimetric function implemented in the macroscopic particle transport simulation code PHITS", International Journal of Radiation Biology 88, 143-150 (2012).

${ }^{86}$ H. Tomita, M. Kai, T. Kusama, and A. Ito, "Monte carlo simulation of physicochemical processes of liquid water radiolysis", Radiation and Environmental Biophysics 36, 105-116 (1997).

${ }^{87}$ Y. Kase, T. Kanai, Y. Matsumoto, Y. Furusawa, H. Okamoto, T. Asaba, M. Sakama, and H. Shinoda, "Microdosimetric measurements and estimation of human cell survival for heavy-ion beams", Radiation Research 166, 629-638 (2006).

${ }^{88}$ A. Zabihi, S. Incerti, Z. Francis, G. Forozani, F. Semsarha, A. Moslehi, P. Rezaeian, and M. Bernal, "Computational approach to determine fast neutrons RBE using the GEANT4-DNA toolkit and a DNA atomic model from the Protein Data Bank", Physical Review E 99, 052404 (2019).

${ }^{89}$ ICRU, Tissue Substitutes in Radiation Dosimetry and Measurement, tech. rep. 44 (ICRU, 1989). 
${ }^{90}$ M. Chadwick, M. Herman, P. Obložinský, M. Dunn, Y. Danon, A. Kahler, D. Smith, B. Pritychenko, G. Arbanas, and R. Arcilla, "ENDF/B-VII.1 Nuclear Data for Science and Technology: Cross Sections, Covariances, Fission Product Yields and Decay Data", Nuclear Data Sheets 112 (2011).

${ }^{91}$ D. Cullen, J. Hubbell, and L. Kissel, EPDL97: the evaluated photo data library 97 version, tech. rep. (Lawrence Livermore National Lab., CA, 1997).

${ }^{92}$ S. Perkins, D. Cullen, and S. Seltzer, "Tables and graphs of electron-interaction cross-sections from $10 \mathrm{eV}$ to $100 \mathrm{GeV}$ derived from the LLNL evaluated electron data library (EEDL), Z=1-100", UCRL-50400 31, 21-24 (1991).

${ }^{93}$ S. Perkins, D. Cullen, M. Chen, J. Rathkopf, J. Scofield, and J. Hubbell, Tables and graphs of atomic subshell and relaxation data derived from the LLNL Evaluated Atomic Data Library (EADL), $Z=1-100$, tech. rep. (Lawrence Livermore National Lab., CA, 1991).

94 ICRP, "Conversion Coefficients for use in Radiological Protection against External Radiation. ICRP Publication 74", Annals of the ICRP 26 (1996).

${ }^{95}$ D. Emfietzoglou, "Inelastic cross-sections for electron transport in liquid water: a comparison of dielectric models", Radiation Physics and Chemistry 66, 373-385 (2003).

${ }^{96}$ D. Emfietzoglou, and H. Nikjoo, "The effect of model approximations on singlecollision distributions of low-energy electrons in liquid water", Radiation Research 163, 98-111 (2005).

${ }^{97}$ I. Kyriakou, S. Incerti, and Z. Francis, "Improvements in Geant4 energy-loss model and the effect on low-energy electron transport in liquid water", Medical Physics $\mathbf{4 2}$, 3870-3876 (2015).

${ }^{98}$ S. Uehara, H. Nikjoo, and D. Goodhead, "Cross-sections for water vapour for the Monte Carlo electron track structure code from $10 \mathrm{eV}$ to the $\mathrm{MeV}$ region", Physics in Medicine and Biology 38, 1841 (1993).

${ }^{99}$ C. Champion, S. Incerti, H. Aouchiche, and D. Oubaziz, "A free-parameter theoretical model for describing the electron elastic scattering in water in the Geant4 toolkit", Radiation Physics and Chemistry 78, 745-750 (2009).

${ }^{100}$ M. Michaud, A. Wen, and L. Sanche, "Cross sections for low-energy (1-100 ev) electron elastic and inelastic scattering in amorphous ice", Radiation Research 159, 3-22 (2003).

${ }^{101} \mathrm{C}$. Melton, "Cross sections and interpretation of dissociative attachment reactions producing $\mathrm{OH}-, \mathrm{O}-$, and $\mathrm{H}-$ in $\mathrm{H}_{2} \mathrm{O}$ ", 
${ }^{102}$ H. Tran, Z. El Bitar, C. Champion, M. Karamitros, M. Bernal, Z. Francis, V. Ivantchenko, S. Lee, J. Shin, S. Incerti, and the Geant4-DNA Collaboration, "Modeling proton and alpha elastic scattering in liquid water in Geant4-DNA", Nuclear Instruments and Methods in Physics Research Section B 343, 132-137 (2015).

${ }^{103}$ M. Dingfelder, M. Inokuti, and H Paretzke, "Inelastic-collision cross sections of liquid water for interactions of energetic protons", Radiation Physics and Chemistry 59, $255-275$ (2000).

${ }^{104}$ M. Rudd, T. Goffe, R. DuBois, and L. Toburen, "Cross sections for ionization of water vapor by 7-4000-keV protons", Physical Review A 31, 492 (1985).

${ }^{105}$ J. Miller, and A. Green, "Proton energy degradation in water vapor",

${ }^{106}$ R Caswell, and J Coyne, "Interaction of Neutrons and Secondary Charged Particles with Tissue: Secondary Particle Spectra", Radiation Research 52 (1972).

${ }^{107}$ Z Francis, S Incerti, V Ivanchenko, C Champion, M Karamitros, M. Bernal, and Z El Bitar, "Monte Carlo simulation of energy-deposit clustering for ions of the same LET in liquid water", Physics in Medicine and Biology 57, 209 (2011).

${ }^{108}$ A. Kellerer, and D. Chmelevsky, "Concepts of microdosimetry. III. Mean values of the Microdosimetric Distributions", Radiation and Environmental Biophysicscs 12, 321-335 (1975).

${ }^{109}$ R. Taleei, P. Girard, and H. Nikjoo, "DSB repair model for mammalian cells in early S and G1 phases of the cell cycle: Application to damage induced by ionizing radiation of different quality", Mutation Research/Genetic Toxicology and Environmental Mutagenesis 779, 5-14 (2015).

${ }^{110}$ S. McMahon, J. Schuemann, H. Paganetti, and K. Prise, "Mechanistic modelling of dna repair and cellular survival following radiation-induced dna damage", Scientific Reports 6, 33290 (2016).

${ }^{111}$ F. Cazals, K. Harshad, and S. Loriot, Computing the Volume of a Union of Balls: a Certified Algorithm, tech. rep. RR-7013 (INRIA, 2009). 